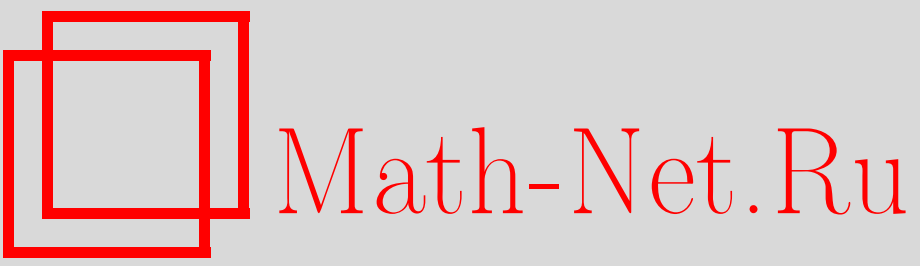

А. В. Пажитнов, Простой гомотопический тип комплекса Новикова и $\zeta$-функция Лефшеца градиентного потока, УМH, 1999, том 54, выпуск 1, 117-170

DOI: https://doi.org/10.4213/rm118

Использование Общероссийского математического портала Math-Net.Ru подразумевает, что вы прочитали и согласны с пользовательским соглашением

http://www . mathnet.ru/rus/agreement

Параметры загрузки:

IP : 3.81 .55 .215

26 апреля 2023 г., 17:36:07 


\title{
ПРОСТОЙ ГОМОТОПИЧЕСКИЙ ТИП КОМПЛЕКСА НОВИКОВА И $\zeta$-ФУНКЦИЯ ЛЕФШЕЦА ГРАДИЕНТНОГО ПОТОКА
}

\author{
А.В. ПАжитнов
}

СОДЕРЖАНИЕ

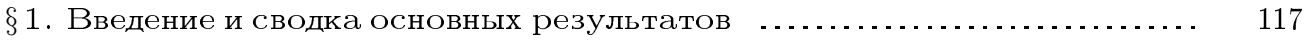

$\S 2$. Теория Морса-Новикова ................................... 121

$\S 3$. Краткий обзор результатов работы $[2]$......................... 127

$\S 4$. Условие $(\mathfrak{C})$ и оператор гомологического градиентного спуска $\quad . . . . .134$

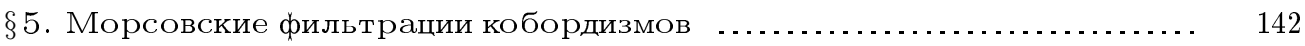

$\S 6$. Алгебраические леммы ...................................... 154

$\S 7$. Условие (드), фильтрации морсовского типа для морсовских отображений $M \rightarrow S^{1}$ и рациональность новиковских коэффициентов

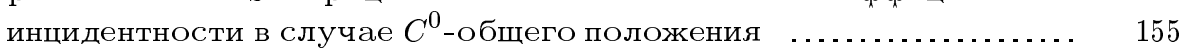

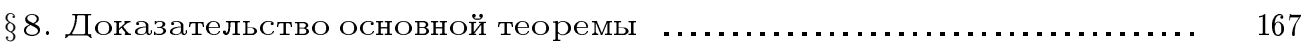

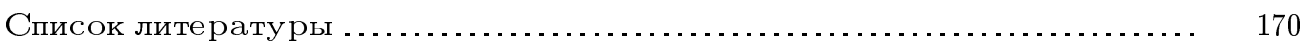

\section{$\S$ 1. Введение и сводка основных результатов}

Основные идеи работы представлены в п. 1.1. Следующие три пункта содержат минимальный набор определений, необходимьй для формулировки основной теоремы, приведенной в п. 1.6. В п. 1.7 дается комментарий к содержанию оставшейся части статьи.

1.1. Введение. В настоящей работе мы продолжаем исследование свойств комплексов Новикова для случая $C^{0}$-обшего положения, которое было начато в работах [1], [2]. Пусть $M$ - замкнутое многообразие. Напомним, что для морсовского отображения $f: M \rightarrow S^{1}$ и $f$-градиента $v$, удовлетворяющего условию трансверсальности, можно построить комплекс Новикова $\mathscr{C}_{*}(v)$ - аналог классического морсовского комплекса. Этот комплекс $\mathscr{C}_{*}(v)$ является свободным конечно-порожденньм цепным комплексом над кольцом $\mathbb{Z}[[t]]\left[t^{-1}\right]$ формальных степенных рядов с конечной отрицательной частью. В цитированных выше работах для данного морсовского отображения $f: M \rightarrow S^{1}$ строится специальный класс $f$-градиентов, образуюший открытое и плотное в $C^{0}$-топологии подмножество в множестве всех $f$-градиентов, удовлетворяющих условию трансверсальности. Для каждого $f$-градиента $v$ в этом классе гра- 
ничные операторы новиковского комплекса, ассоциированные $c$, являются не просто степенными рядами, но рациональными функциями.

Основной конструкцией в доказательстве является гомоморфизм $h(v)$, введенньй в $[2 ; \S 4]$, который мы будем здесь называть оператором гомологического градиентного спуска. (Для того чтобы объяснить смысл этого названия, рассмотрим регулярное значение $\lambda \in S^{1}$ отображения $f$ и рассмотрим интегральную кривую $\gamma$ для $(-v)$, начинаюшуюся в $x \in V=f^{-1}(\lambda)$. Если эта кривая не сходится к критической точке отображения $f$, то тогда в некоторой точке- обозначим ее $\rho(x)$ - она снова пересечется c $V$. Определенное таким образом отображение $\rho$ является гладким отображением из $V$ в $V$. Это отображение $\rho$ определено, быть может, не всюду, поэтому, вообще говоря, не сушествует индуцированного им гомоморфизма в гомологиях $H_{*}(V)$. Однако можно построить некоторьй гомоморфизм групп, который для наших целей является вполне эффективньм заменителем $\rho_{*}$. Этот оператор определяется для $f$-градиента, удовлетворяющего условию трансверсальности и находящегося в $C^{0}$-общем положении.) В настоящей работе продолжается исследование свойств оператора гомологического градиентного спуска. Мы показьваем, что этот гомоморфизм несет достаточно информации для вычислении $\zeta$-функции Лефшеца градиентного потока. Это приводит нас к основной теореме - ее формулировка дана в п. 1.6. Чтобы объяснить вкратце идею основной теоремы, напомним [3], что между комплексом Новикова и пополненным комплексом симплициальных цепей соответствующего циклического накрытия сушествует цепная гомотопическая эквивалентность. Основная теорема утверждает, что для $f$-градиента $v$, находяшегося в $C^{0}$-общем положении, эта цепная гомотопическая эквивалентность может быть выбрана таким образом, что ее кручение равно $\zeta$-функции Лефшеца потока, порожденного $(-v)$.

1.2. Алгебраические понятия: $K_{1}$, кольца Новикова и т. д. Пусть $R$ коммутативное кольцо с единицей. Напомним определение групшы $K_{1}$ из [4]: $\bar{K}_{1} R=K_{1} R /\{0,[-1]\}$, где [-1] обозначает элемент порядка 2 , соответствуюший единице $(-1) \in G L(R, 1) \subset G L(R)$. Далее, $K_{1} R=R^{\bullet} \oplus S K_{1}(R)$, где $R^{\bullet}$ - полная группа единиц кольца $R$. Пусть $U$ - подгруппа в $R^{\bullet}$. Положим $K_{1}(R \mid U)=\bar{K}_{1} R / U=$ $R^{\bullet} /( \pm U) \oplus S K_{1}(R)$ (здесь $\pm U=\{ \pm u \mid u \in U\}$ и мы отождествляем единищы в $R^{\bullet}$ с их образами в $\left.\bar{K}_{1} R\right)$. Для $S \in G L(R)$ образ $S$ в $\bar{K}_{1} R$ или в $K_{1}(R \mid U)$ будет обозначаться посредством $[S]$.

Свободно конечно-порожденный цепной комплекс $C_{*}$ правых $R$-модулей (см. [5; определение 1.3]) назьвается $R$-комплексом.

Напомним $[4 ; \S 3]$, что для ацикличного $R$-комплекса $C_{*}$ можно определить кручение $\tau\left(C_{*}\right) \in \bar{K}_{1} R$.

Если $\phi: F_{*} \rightarrow D_{*}$ - гомотопическая эквивалентность $R$-комплексов, то ее кручение $\tau(\phi) \in \bar{K}_{1} R$ определяется как кручение цепного конуса $C_{*}(\phi)$. Образ $\tau(\phi)$ в групе $K_{1}(R \mid U)$ будет обозначаться посредством $\tau(\phi \mid U)$.

Перейдем к определениям, касающимся новиковских пополнений. Пусть $G$ - абелева группа и $\xi: G \rightarrow \mathbb{R}$ - гомоморфизм. Положим $\Lambda=\mathbb{Z} G$ и обозначим символом $\widehat{\widehat{\Lambda}}$ абелеву группу всех функций $G \rightarrow \mathbb{Z}$. Группу $\widehat{\widehat{\Lambda}}$ можно также определить как множество всех формальных линейных комбинаций $\sum_{g \in G} n_{g} g$ (не обязательно конечных) элементов из $G$ с целыми коэффициентами. Для $\lambda \in \widehat{\widehat{\Lambda}}$ положим supp $\lambda=\left\{g \in G \mid n_{g} \neq 0\right\}$. 
Пусть $\widehat{\Lambda}_{\xi}$ обозначает подгруппу в групе $\widehat{\widehat{\Lambda}}$, состоящую из всех $\lambda$ таких, что для каждого $C>0$ множество $\xi^{-1}\left(\left[C, \infty[) \cap \operatorname{supp} \lambda\right.\right.$ конечно. Тогда $\widehat{\Lambda}_{\xi}$ имеет естественную структуру кольца. Пусть, далее, $\widehat{\widehat{\Lambda}}_{\mathbb{Q}}-$ множество всех функций $G \rightarrow \mathbb{Q}$. Рассмотрим кольцо

$$
\widehat{\Lambda}_{\xi, \mathbb{Q}}=\left\{\lambda \in \widehat{\widehat{\Lambda}}_{\mathbb{Q}} \mid \forall C \in \mathbb{R} \text { множество } \operatorname{supp} \lambda \cap \xi^{-1}([C, \infty[) \text { конечно }\} .\right.
$$

В дальнейшем мы будем иметь дело с двумя подгруппами единиц кольца $\widehat{\Lambda}_{\xi}$. Первая такая подгруппа - сама группа $G$, вторая вводится в следуюшей формуле:

$$
U_{\xi}=\left\{\lambda \in \widehat{\Lambda}_{\xi} \mid \lambda= \pm g(1+\mu) \text {, где } g \in G \text { и } \operatorname{supp} \mu \subset \xi^{-1}(]-\infty, 0[)\right\} .
$$

В [3] группа $K_{1}\left(\widehat{\Lambda}_{\xi} \mid U_{\xi}\right)$ обозначалась символом $W h(G, \xi)$.

Нам понадобится еще одно кольцо. Пусть $S_{\xi}=\{\lambda \in \mathbb{Z} G \mid \lambda=1+\mu, \operatorname{supp} \mu \subset$ $\left.\left.\left.\xi^{-1}(]-\infty, 0\right]\right)\right\}$. Обозначим $S_{\xi}^{-1} \Lambda$ также символом $\Lambda_{(\xi)}$ и отметим, что имеется естественное вложение $\Lambda \hookrightarrow \Lambda_{(\xi)} \hookrightarrow \widehat{\Lambda}_{\xi}$.

1.3. Комплекс Новикова. Здесь мы скажем лишш, что это такое; определения и мотивировки см. в п. 2.4.

Пусть $f: M \rightarrow S^{1}$ - морсовское отображение. Мы предполагаем, что $f_{*}: H_{1}(M) \rightarrow$ $H_{1}\left(S^{1}\right)=\mathbb{Z}$ - эпиморфизм. Пусть $\mathscr{P}: \widetilde{M} \rightarrow M$ - регулярное связное накрытие со структурной группой $G$ такое, что $f \circ \mathscr{P}$ гомотопно нулю. ${ }^{1}$ В этой работе мы рассматриваем только случай, когда группа $G$ абелева. В этом случае имеется естественный эпиморфизм $\pi: H_{1}(M) \rightarrow G$ и $f_{*}: H_{1}(M) \rightarrow \mathbb{Z}$ пропускается через гомоморфизм $\xi: G \rightarrow \mathbb{Z}$. Положим $\Lambda=\mathbb{Z} G$, тогда определено соответствуюшее кольцо Новикова $\widehat{\Lambda}_{\xi}$. Пусть $v$ есть $f$-градиент, удовлетворяюший условию трансверсальности (определение см. в п. 2.1). В каждой критической точке $p$ отображения $f$ выберем ориентацию устойчивого многообразия точки $p$ и поднятие $p$ в $\widetilde{M}$.

По этим данным и строится новиковский комплекс $\widetilde{\mathscr{C}}_{*}(v)$ : это свободный $\widehat{\Lambda}_{\xi \text {-комп- }}$ лекс, у которого число свободных образующих модуля $\widetilde{\mathscr{C}}_{k}(v)$ равно числу критических точек индекса $k$ отображения $f$.

1.4. Потоки и орбиты. Пусть $w$ есть $C^{\infty}$-векторное поле на замкнутом многообразии $M$. Замкнутая орбита поля $w$-это траектория $\gamma:[a, b] \rightarrow M$ поля $w$, не являюшаяся постоянным отображением и удовлетворяюшая условию $\gamma(a)=\gamma(b)$. Мы будем отождествлять две такие траектории $\gamma:[a, b] \rightarrow M, \gamma^{\prime}:\left[a^{\prime}, b^{\prime}\right] \rightarrow M$, если найдется такое $C$, что $a^{\prime}=a+C, b^{\prime}=b+C$ и $\gamma(t)=\gamma^{\prime}(t+C)$.

Множество всех замкнутых орбит обозначается $\mathrm{Cl}(w)$. Пусть $\gamma \in \mathrm{Cl}(w), \gamma:[a, b] \rightarrow$ $M$; пусть $m \in \mathbb{N}$. Результат последовательного $m$-кратного прохождения траектории $\gamma$ есть замкнутая орбита, которую мы будем обозначать $\gamma^{m}$. Kратность орби$m b l \gamma \in \mathrm{Cl}(w)$ - это наибольшее число $k \in \mathbb{N}$ такое, что $\gamma=\theta^{m}$ для некоторой орбиты $\theta \in \mathrm{Cl}(w)$. Для любой орбиты $\gamma \in \mathrm{Cl}(w)$ определен ее класс гомологий $[\gamma] \in H_{1}(M)$. Векторное поле $w$ назьвается полем Купки-Смейл $а$, если любой нуль и любая замкнутая орбита поля $w$ являются гиперболическими и если $w$ удовлетворяет условию

\footnotetext{
${ }^{1}$ Таким образом, символ , обычно используемый для обозначения универсального покрытия, мы используем для других целей.
} 
трансверсальности. Если $w$ есть поле Купки-Смейла, то для любой орбиты $\gamma \in \mathrm{Cl}(w)$ определен ее индекс $\varepsilon(\gamma) \in\{-1,1\}$ (это индекс соответствуюшего отображения Пуанкаре).

1.5. $\zeta$-функция Лефшеца. Мы будем рассматривать $\zeta$-функции только для очень специального класса потоков, а именно, для потоков на замкнутых многообразиях, порожденных градиентами морсовских отображений $f: M \rightarrow S^{1}$.

Множество всех градиентов Купки-Смейла данного морсовского отображения $f: M \rightarrow S^{1}$ обозначается символом $\mathscr{G} \mathscr{K} \mathscr{S}(f)$. Пусть $v \in \mathscr{G} \mathscr{K} \mathscr{S}(f)$. Для каждой замкнутой орбиты $\gamma \in \mathrm{Cl}(-v)$ рассмотрим элемент $\pi([\gamma])$ как элемент группового кольца $\Lambda=\mathbb{Q} G$, умножим этот элемент на $\frac{\varepsilon(\gamma)}{m(\gamma)}$ и рассмотрим бесконечньй ряд

$$
\eta_{L}(-v)=\sum_{\gamma \in \mathrm{Cl}(-v)} \frac{\varepsilon(\gamma)}{m(\gamma)} \pi([\gamma])
$$

Из свойства Купки-Смейла вытекает, что для каждого $n \in \mathbb{Z}$ число орбит $\gamma$, удовлетворяюших $f_{*}([\gamma])=n$, конечно. Таким образом, этот ряд действительно принадлежит $\widehat{\widehat{\Lambda}}_{\mathbb{Q}}$. Несложно показать, что $\eta_{L}(-v)$ принадлежит кольцу Новикова $\widehat{\Lambda}_{\xi, \mathbb{Q}}$. Заметим также, что $\left.\left.\operatorname{supp} \eta_{L}(-v) \subset \xi^{-1}(]-\infty,-1\right]\right)$. Поэтому $\operatorname{supp}\left(\eta_{L}(-v)\right)^{k} \subset$ $\left.\left.\xi^{-1}(]-\infty,-k\right]\right)$ и бесконечный ряд

$$
\zeta_{L}(-v)=\exp \left(\eta_{L}(-v)\right)
$$

корректно определен и снова принадлежит $\widehat{\Lambda}_{\xi, \mathbb{Q}}$. Этот элемент и называется $\zeta$ - функиией Лефшеца.

1.6. Формулировка основной теоремы. Пусть $M$ - замкнутое связное многообразие, $f: M \rightarrow S^{1}$ - морсовское отображение. Мы будем использовать обозначения из п. 1.3. Пусть $\lambda$ - единица кольца $\Lambda_{(\xi)}$, ее образ в $\bar{K}_{1}\left(\Lambda_{(\xi)} \mid G\right)$ будем обозначать $\bar{\lambda}$. Символом $C_{*}^{\Delta}(\widetilde{M})$ мы обозначаем комплекс симплициальных цепей пространства $\widetilde{M}$, ассоциированньй с гладкой триангуляцией $M\left(\right.$ так что $C_{*}^{\Delta}(\widetilde{M})-\mathbb{Z} G$-комплекс $)$.

TeOpema. Существует подмножество $\mathscr{G} \mathscr{K} \mathscr{S}(f) \subset \mathscr{G} \mathscr{K} \mathscr{S}(f)$ со следующими свойствами.

1. $\mathscr{G} \mathscr{K} \mathscr{S}(f)$ - открытое и плотное подмножество $\mathscr{G} \mathscr{K} \mathscr{S}(f)$ в смысле $C^{0}$-топологии.

2. Для кажсдого $v \in \mathscr{G} \mathscr{K} \mathscr{S} \mathfrak{C}(f)$ комплекс Новикова $\widetilde{\mathscr{C}}_{*}(v)$ определен над $\Lambda_{(\xi)}$, $u \zeta_{L}(-v) \in \Lambda_{(\xi)}$.

3. Для каждого $v \in \mathscr{G} \mathscr{K} \mathscr{C}(f)$ существует гомотопическая әквивалентность

$$
\phi: \widetilde{\mathscr{C}}_{*}(v) \stackrel{\sim}{\longrightarrow} C_{*}^{\Delta}(\widetilde{M}) \underset{\Lambda}{\otimes} \Lambda_{(\xi)}
$$

такая, что $\tau(\phi \mid G)=\overline{\zeta_{L}(-v)}$. 
1.7. Замечания и комментарии. Впервые результаты работы появились в электронном препринте [6]. Настояшая работа содержит детальное изложение результатов [6] (за недостатком времени мы не включили результаты [6], касающиеся иррациональных морсовых форм и связи с инвариантами Зайберга-Виттена трехмерных многообразий).

В пп. 2.1-2.3 2 мы даем необходимые для дальнейшего определения. В пп. 2.4 и 2.5 дается введение в теорию Морса-Новикова и описание результатов автора, касаюшихся гипотезы Новикова об экспоненциальном росте ([1], [2]); причем представлены скорее идеи, нежели технические подробности.

Параграф 3 содержит краткий обзор, касающийся технических деталей и результатов работы [2], которые потребуются нам в дальнейшем. Эта часть включена в работу, чтобы сделать ее максимально независимой от других источников (доказательства могут быть найдены, например, в [2]). Содержание $\S 4$ параллельно содержанию $\S 4$ в [2]; условие (ㄷ) (п. 4.2) аналогично условию (RP) в 44 работы [2].

Одним из центральных разделов работы является $\S 5$; основные идеи представлены в его начале.

В $§ 6$ содержатся несложные алгебраические выгисления. Параграф 7 содержит наиболее важные шаги доказательства основной теоремы: в терминах морсовских фильтраций кобордизмов вычисляется простой гомотопический тип комплекса Новикова (введенньй в $\S 5$ ) и связанные с ним инварианты. В $\S 8$ завершается доказательство основной теоремы.

Мы закончим вводную часть замечанием по поводу истоков данной работы. Используя методы работы [2], несложно показать, что $\zeta$-функция Лефшеца для градиента, находящегося в $C^{0}$-обшем положении, является рациональной, однако не ясно, как ее вычислить в терминах обычных инвариантов многообразий. Первым примером такого вычисления была формула Хатчингса-Ли ([7; теорема 1.1]). Эта формула дала основания предполагать, что в общем случае должна быть формула, связывающая простой гомотопический тип комплекса Новикова с простым гомотопическим типом многообразия и $\zeta$-функцией градиентного потока.

Автор признателен С. П. Новикову, Б. Л. Вану и В. Г. Тураеву за ценные обсуждения.

Первоначальный вариант настояшего текста был написан по-английски. Перевод на русский язык выполнен А. Алимовым.

\section{§. Теория Морса-Новикова}

2.1. Функции Морса и их градиенты: основные понятия. Прежде чем углубляться в детали теории Морса-Новикова и гипотезы Новикова об экспоненциальном росте, мы дадим основные определения из теории Морса, которыми мы будем пользоваться в дальнейшем.

Пусть $v$ есть $C^{1}$-гладкое векторное поле на многообразии $M$. Значение интегральной кривой поля $v$, проходящей через $x$ при $t=0$, будет обозначаться символом $\gamma(x, t ; v)$.

Кобордизмом мы называем компактное многообразие $W$ вместе с представлением $\partial W=\partial_{0} W \sqcup \partial_{1} W$, где $\partial_{1} W$ и $\partial_{0} W$ - компактные многообразия без границы, размерности $\operatorname{dim} W-1$ (оба или одно из них могут быть пустыми). Многообразие $W \backslash \partial W$ 
будет обозначаться символом $W^{\circ}$.

Функцией Морса $f: W \rightarrow[a, b]$ на кобордизме $W$ назьвается $C^{\infty}$-гладкое отображение $f: W \rightarrow \mathbb{R}$ такое, что $f(W) \subset[a, b], f^{-1}(b)=\partial_{1} W, f^{-1}(a)=\partial_{0} W$, все критические точки $f$ являются невырожденными и принадлежат $W^{\circ}$.

Множество всех критических точек функции Морса $f$ будет обозначаться символом $S(f)$; а множество всех критических точек индекса $k$ для $f$-символом $S_{k}(f)$.

Пусть $f: W \rightarrow \mathbb{R}$ - функция Морса на кобордизме $W, \operatorname{dim} W=n$. Векторное поле $v$ назьвается градиентом функции $f$ или $f$-градиентом, если для каждой критической точки $p$ функции $f$ найдется карта $\phi: U \rightarrow V$, содержащая $p$, такая, что $\phi_{*}(v)$ является стандартным векторным полем $\left(-x_{1}, \ldots,-x_{k}, x_{k+1}, \ldots, x_{n}\right)$ на $\mathbb{R}^{n}$ (где $k=$ ind $p$ ) и $f \circ \phi^{-1}-$ квадратичная форма $\sum_{i} \alpha_{i} x_{i}^{2}$ с $\alpha_{i}>0$ при $i>k$ и $\alpha_{i}<0$ при $i \leqslant k$.

Множество всех $f$-градиентов мы обозначаем $\mathscr{G}(f)$.

\section{ЗАМЕЧАНИЕ 2.1.}

1. Нетрудно проверить, что если в данном определении мы потребуем $\left\|\alpha_{i}\right\|=1$, то получится эквивалентное определение.

2. Риманов градиент функции $f$ (по отношению к некоторой римановой метрике) не является, вообще говоря, $f$-градиентом в смысле нашего определения. Можно, однако, расширить определение, чтобы устранить этот недостаток и обобшить соответствуюшим образом результаты настояшей работы (см. [8]).

Пусть $f: W \rightarrow[a, b]$ есть функция Морса на кобордизме $W$ размерности $n$, а $v$ есть $f$-градиент.

Символом $K_{1}$ обозначим множество всех $x \in \partial_{1} W$ таких, что $(-v)$-траектория, начинающаяся в $x$, сходится к критической точке функции $f$. Аналогично, $K_{0}$ обозначает множество всех $x \in \partial_{0} W$ таких, что $\gamma(x, t ; v)$ сходится к критической точке $f$. Заметим, что $K_{0}, K_{1}$ компактны. Сдвиг вдоль траекторий $(-v)$ определяет диффеоморфизм $\partial_{1} W \backslash K_{1} \rightarrow \partial_{0} W \backslash K_{0}$, который мы будем обозначать символом $(-v)^{\rightsquigarrow}$.

Пусть $A \subset K_{1}$. Вместо символа $(-v)^{\rightsquigarrow}\left(A \backslash K_{1}\right)$ мы будет употреблять для краткости символ $(-v)^{\rightsquigarrow}(A)$.

Пусть $\lambda, \mu, \lambda<\mu,-$ регулярные значения $f$. Положим $W^{\prime}=f^{-1}([\lambda, \mu])$ и $w=$ $v \mid W^{\prime}$. Диффеоморфизм $(-w)^{\rightsquigarrow}$ будет обозначаться $(-v)_{[\mu, \lambda]}^{\rightsquigarrow}$.

Нам потребуется одна стандартная конструкция из теории Морса (см. [9; с. 62]). Пусть $\Psi: \partial_{0} W \times[0, l] \rightarrow W$ - отображение, задаваемое формулой $\Psi(x, t)=\gamma(x, t ; v)$. Выберем $l$ достаточно малым, так, что $\Psi$ - диффеоморфизм на свой образ. Пусть $h-C^{\infty}$-гладкая положительная функция на $\left.[0, l], \operatorname{supp} h \subset\right] 0, l[$. Положим $T=\int_{0}^{l} h(\tau) d \tau$. Пусть $u$ - векторное поле на $\partial_{0} W$. Определим векторное поле $w$ на $\partial_{0} W \times[0, l]$, положив $w(x, t)=h(t) u(x)$. Определим векторное поле $w^{\prime}$ на $W$, положив $w^{\prime}=\Psi_{*}(w)$ в $\operatorname{Im} \Psi$ и $w^{\prime}=0$ в $W \backslash \operatorname{Im} \Psi$. Пусть, далее, $v^{\prime}=v+w^{\prime}$. Если $h$ и $u$ достаточно малы, то $\left\|v-v^{\prime}\right\|$ мало, и $v^{\prime}$ остается $f$-градиентом. Заметим, что $\left(-v^{\prime}\right)^{\rightsquigarrow}=\Phi(-u, T) \circ(-v)^{\rightsquigarrow}$. Мы будем называть эту конструкцию “операцией прибавления горизонтальной компоненты $u$ около $\partial_{0} W^{\prime \prime}$.

Скажем, что $v$ удовлетворяет условию трансверсальности, если

$$
(x, y \in S(f)) \Rightarrow\left(D(x, v) \cap W^{\circ} \pitchfork D(y,-v) \cap W^{\circ}\right) .
$$


Скажем, что $v$ удовлетворяет условию почти-трансверсальности, если

$$
(x, y \in S(f) \& \text { ind } x \leqslant \text { ind } y) \Rightarrow\left(D(x, v) \cap W^{\circ} \pitchfork D(y,-v) \cap W^{\circ}\right) .
$$

Множество всех $f$-градиентов, удовлетворяющих условию трансверсальности (соответственно, условию почти-трансверсальности), будет обозначаться посредством $\mathscr{G} \mathscr{T}(f)$ (соответственно $\mathscr{G} \mathscr{A}(f))$.

Функция Морса $\phi: W \rightarrow[\alpha, \beta]$ назьвается согласованной $c(f, v)$, если:

1) $S(\phi)=S(f)$ и $v$ - также $\phi$-градиент;

2 ) функция $f-\phi$ постоянна в окрестности $\partial_{0} W$, в окрестности $\partial_{1} W$ и в окрестности каждой точки из $S(f)$.

Мы называем $f$ упорядоченной функцией Морса с упорядочивающей последовательностью $\left(a_{0}, \ldots, a_{n+1}\right)$, если $a=a_{0}<a_{1}<\cdots<a_{n+1}=b$ являются регулярными значениями функции $f$ такими, что $S_{i}(f) \subset f^{-1}(] a_{i}, a_{i+1}[)$.

2.2. Еще о терминологии. Мы придерживаемся соглашения, что структурная группа регулярного накрытия действует на накрытии справа. Все рассматриваемые в работе регулярные накрытия будут иметь коммутативную структурную группу (однако существенная часть наших результатов может быть перенесена без проблем и на неабелев случай).

2.3. Морсовские комплексы функций. Прежде чем перейти к изложению теории Морса-Новикова, напомним вкратце классическую конструкцию комплекса Морса. Пусть $g: M \rightarrow \mathbb{R}$ - функция Морса на замкнутом многообразии $M$. В классической теории Морса [10] доказывается, что $m_{p}(g) \geqslant b_{p}(M)$, где $m_{p}(g)$ - число критических точек индекса $p$ и $b_{p}(M)-p$-е число Бетти многообразия $M$. Это неравенство можно вывести из существования цепного комплекса $C_{*}$ свободных абелевых групп такого, что число $\mu\left(C_{p}\right)$ свободных образуюших $C_{p}$ равно $m_{p}(g)$ и $H_{*}\left(C_{*}\right) \approx H_{*}(M)$. Этот комплекс, называемьй комплексом Морса, был построен позже работы [10]. Его построение есть результат исследований нескольких авторов. Основной шаг был сделан в работах Р. Тома [11] и С. Смейла [12]. В книге Дж. Милнора [9] дается систематическое изложение этого шага. (Фактически, однако, в указанной книге нет определения самого комплекса, но это определение может быть получено из $\S 6$ книги.) Позже эта конструкция была рассмотрена Виттеном [13] с принципиально новой точки зрения, включающей когомологии Де Рама.

Вот основная идея конструкции. Выберем $g$-градиент $v$, удовлетворяющий условию трансверсальности. Пусть $C_{s}-$ свободная абелева группа, порожденная критическими точками функции $g$ индекса $s$. Пусть $p, q$ - критические точки функции $g$ индексов $k$ и $k-1$ соответственно. Так как $v$ удовлетворяет условию трансверсальности, множество $L(p, q)$ всех орбит, соединяющих $p$ с $q$, конечно. В каждой критической точке $g$ выберем ориентацию устойчивого многообразия (по отношению к векторному полю $v)$. Тогда каждой орбите $\gamma \in L(p, q)$ может быть некоторым естественным образом приписан знак $\varepsilon(\gamma)$. Положим $n(p, q)=\sum_{\gamma \in L(p, q)} \varepsilon(\gamma)$. Граница $\partial_{k}: C_{k} \rightarrow C_{k-1}$ определяется формулой $\partial_{k} p=\sum_{q} n(p, q) q$ (где $q$ пробегает множество всех критических точек функции $g$ индекса $k-1)$.

Оказывается, что $\partial_{k} \circ \partial_{k+1}=0$ для каждого $k$ и что гомологии комплекса $C_{*}$ по отношению к $\partial_{*}$ изоморфны $H_{*}(M)$ (детали можно найти в [3; приложение]). Этот комплекс называется комплексом Морса. 
2.4. Краткое введение в теорию Морса-Новикова. В работе [14] С.П. Новиков заложил основы теории Морса для замкнутых 1-форм. Одним из основных понятий в этой теории является понятие комплекса Новикова. Здесь мы дадим лишь краткое описание конструкции (подробное изложение дано в [3]).

Рассмотрим морсовское отображение $f: M \rightarrow S^{1}$. Конструкция из п. 2.3 не может быть перенесена на этот случай, так как для двух критических точек $x, y$ соседних индексов число орбит градиента $v$, соединяюших $x$ с $y$, может быть бесконечным. Вот как можно обойти эту трудность: разобьем множество всех орбит, соединяющих $x$ с $y$, на некоторое семейство непересекающихся конечных подмножеств (перенумерованное индексами некоторого множества $I$ ), посчитаем отдельно орбиты в каждом классе и таким образом получим коэффицциент инцидентности как функцию $I \rightarrow \mathbb{Z}$. Таким образом, кольцо, над которым определен получающийся комплекс, будет бо́льшим, нежели $\mathbb{Z}$. Мы опишем наиболее естественньй способ такого разбиения (он введен в [14]); он приводит к основному кольцу $\mathbb{Z}((t))$.

ОпРЕДЕЛЕНИЕ 2.2. Кольцо $\mathbb{Z}((t))$ состоит из степенных рядов по $t$ с целыми коэффициентами и конечной отрицательной частью. То есть $\lambda=\sum_{-\infty}^{\infty} a_{i} t^{i}$ лежит в $\mathbb{Z}((t))$, если $a_{i} \in \mathbb{Z}$ для всех $i$ и найдется $N=N(\lambda)$ такое, что $a_{i}=0$ при $i<N(\lambda)$.

Мы будем предполагать, что морсовское отображение $f$ не гомотопно нулю. В противном случае $v$ является градиентом обычной вешественнозначной функции Морса и применима конструкция из п. 2.3. Пусть $\mathscr{C}_{p}(f)$ - свободный $\mathbb{Z}((t))$-модуль, порожденньй критическими точками отображения $f$ индекса $p$. Рассмотрим бесконечное циклическое накрытие $\mathscr{C}: \bar{M} \rightarrow M$ такое, что $f$ ० $\mathscr{C}$ гомотопно нулю. Поднимем функцию $f: M \rightarrow S^{1}$ до функции $F: \bar{M} \rightarrow \mathbb{R}$. Пусть $t$ - образуюшая структурной группы накрытия $\mathscr{C}$ такая, что $F(x t)<F(x)$. Выберем градиент $v \in \mathscr{G} \mathscr{T}(f)$ и поднимем его в $\bar{M}$ (мы сохраняем для этого поднятия прежнее обозначение $v$ ). Как и выше, для каждой критической точки $x \in S(f)$ выберем ориентацию устойчивого многообразия точки $x$ по отношению к $v$. Более того, для каждой критической точки $x$ отображения $f: M \rightarrow S^{1}$ выберем поднятие $\bar{x}$ точки $x$ в $\bar{M}$. Пусть $x, y$ - две критические точки функции $f$ и ind $x=$ ind $y+1$. Несложно показать, что для любого $k \in \mathbb{Z}$ множество всех соединяюших $\bar{x}$ и $\bar{y} t^{k} v$-орбит конечно. Просуммируем их с соответствуюшими знаками (как и выше) и получим целое число $n_{k}(x, y)$. Положим $n(x, y)=\sum_{k} n_{k}(x, y) t^{k} \in \mathbb{Z}((t))$ и $\partial_{k} x=\sum_{y} y \cdot n(x, y)$. (Степенные ряды $n(x, y)$ называются коәффициентами инцидентности Новикова. Заметим, что $n(x, y)$ и $n_{k}(x, y)$ зависят от $v$ и иногда мы будем писать $n(x, y ; v)$ и, соответственно, $n_{k}(x, y ; v)$, чтобы подчеркнуть эту зависимость.) Заметим, что комплекс Новикова для нас является комплексом правых модулей (см. п. 2.2).

Можно показать, что $\partial_{k} \circ \partial_{k+1}=0$. Итак, мы получили цепной комплекс свободных конечно-порожденных $\mathbb{Z}((t))$-модулей. Мы обозначим его $\mathscr{C}_{*}(v)$, чтобы подчеркнуть его зависимость от $v$, которая сушественна для наших дальнейших целей. ${ }^{2}$ (В действительности этот комплекс зависит также и от выбора ориентаций на устойчивых многообразиях критических точек $f$, и от поднятия критических точек в $\bar{M}$, но влияние этого выбора на результат менее значительно: неопределенность сводится к умножению строк и столбцов матриц граничных операторов $\partial_{k}$ на некоторые единицы

\footnotetext{
${ }^{2}$ Мы выбрали рукописное написание $\mathscr{C}$, чтобы различать комплексы Новикова и Морса.
} 
вида $\pm t^{m}$ основного кольца. С другой стороны, ясно, что этот комплекс не зависит от конкретного выбора функции $f$, для которой $v$ является градиентом.) Чтобы описать гомологии комплекса, напомним, что $H_{*}(\bar{M})$ является $\mathbb{Z}\left[t, t^{-1}\right]$-модулем, так что тензорное произведение $\widehat{H}_{*}(\bar{M})=H_{*}(\bar{M}) \underset{\mathbb{Z}\left[t, t^{-1}\right]}{\otimes} \mathbb{Z}((t))$ имеет смысл. Можно показать, что $H_{*}\left(\mathscr{C}_{*}(v)\right) \approx \widehat{H}_{*}(\bar{M})$.

Все упомянутые результаты о комплексе Новикова сформулированы в [14]. Подробные доказательства содержатся в [3].

2.5. Гипотеза Новикова об экспоненциальном росте и оператор гомологического градиентного спуска. Пусть $f: M \rightarrow S^{1}$ - морсовское отображение, и пусть $v$ есть $f$-градиент, удовлетворяющий условию трансверсальности. В предыдущем пункте мы видели, что в этом случае определен комплекс Новикова $\mathscr{C}_{*}(v)$. Он является свободным цепньм комплексом над $\mathbb{Z}((t))$ с фиксированным базисом. Следовательно, каждый граничный оператор $\partial_{k}$ представляется матрищей $D^{(k)}$ с элементами $D_{i j}^{(k)}$ из $\mathbb{Z}((t))$ (ее элементы суть не что иное, как новиковские коэффициенты инцидентности). Каждый элемент $D_{i j}^{(k)}$ является степенным рядом $a(t)=\sum_{k} a_{k} t^{k}$ с конечной отрищательной частью. Гипотеза Новикова об экспоненциальном росте утверждает, что коэффициенты $a_{k}$ растут не более чем с экспоненциальной скоростью при $k \rightarrow \infty$ (возможно, при некоторых ограничениях на $v$; например, типа аналитичности или общности положения). Более подробно об этой гипотезе см. введение к работе [2]. Эта гипотеза была доказана в [2] для произвольного морсовского отображения $f: M \rightarrow S^{1}$ и множества $\mathscr{G}_{0}(f) f$-градиентов, которое открыто и плотно в смысле $C^{0}$-топологии в $\mathscr{G}(f)$. Для градиентов, принадлежащих $\mathscr{G} \mathscr{T}_{0}(f)$, новиковские коэффициенты инцидентности являются рядами Тейлора некоторых рациональных функций. Доказательство рациональности коэффициентов инцидентности для $v \in \mathscr{G}_{0}(f)$ основано на конструкции, которую мы назьваем оператором гомологического градиентного спуска. Эта конструкция очень важна для настоящей работы, так что остальную часть п. 2.5 мы посвятим объяснению основной идеи этой конструкции. Подробное изложение см. в пा. 3.5, 4.3.

Предположим, что гомотопический класс отображения $f$ в $\left[M, S^{1}\right] \approx H^{1}(M, \mathbb{Z})$ неделим. Пусть $p, q$ - критические точки функции $f$, ind $p=\operatorname{ind} q+1$. Мы будем рассматривать новиковские коэффициенты инцидентности $n(p, q ; v)$. Для удобства обозначений предположим, что $1 \in S^{1}$ - регулярное значение $f$, первьй критический уровень после $f(q)$ в направлении против часовой стрелки есть $f(p)$ и 1 содержится между $f(q)$ и $f(p)$.

Разрежем $M$ вдоль $f^{-1}(1)$ и получим кобордизм $W$ с двумя частями границы: $\partial_{1} W$ и $\partial_{0} W$. Имеется диффеоморфизм отождествления $\Phi: \partial_{0} W \rightarrow \partial_{1} W$. Имеется также функция Морса $f_{0}: W \rightarrow[0,1]$ и ее градиент, которьй будет обозначаться той же буквой $v$. Заметим, что $p$ - низшая критическая точка $f_{0}$, а $q$ - наивысшая. Нисходяший диск точки $p$ пересекает $\partial_{0} W$ по вложенной сфере $S(p)$. Восходящий диск точки $q$ пересекает $\partial_{1} W$ по вложенной сфере $S(q)$. Тогда новиковский коэффициент инцидентности $n_{k}=n_{k}(p, q ; v)$ - это алгебраический индекс пересечения $\left((-v)^{\rightsquigarrow} \circ \Phi\right)^{k}(S(p))$ с $\Phi^{-1}(S(q)){ }^{3}$ Положим $\phi=(-v)^{\rightsquigarrow} \circ \Phi$. Если бы $\phi$ был везде определенным диффеоморфизмом, тогда, обозначая классы гомологий $S(p)$ и $\Phi^{-1}(S(q))$ через $[p]$ и $[q]$

\footnotetext{
${ }^{3}$ См. с. 122 по поводу определения $(-v)^{\rightsquigarrow}$.
} 
соответственно, мы бы получили формулу

$$
n_{k}(p, q ; v)=\left(\phi_{*}\right)^{k}([p]) \#[q]
$$

(где \# обозначает алгебраический индекс пересечения классов гомологий; мы предполагаем здесь, что $\partial_{0} W$ ориентировано, хотя в действительности ориентируемость не сушественна). В этом случае рациональность степенного ряда $\sum_{k} n_{k} t^{k}$ была бы простым утверждением из линейной алгебры. Для полноты картины приведем здесь это рассуждение. Пусть $L$ - свободная конечно-порожденная абелева группа, $A: L \rightarrow L$ - гомоморфизм. Отождествим $A$ с его матрицей. Тогда степенной ряд $\sum_{k=0}^{\infty} A^{k} t^{k}$ представляет матрищу $(1-A t)^{-1}$. Из этого легко вывести, что

(2) $\quad \sum_{k=0}^{\infty} \xi\left(A^{k} x\right) t^{k}$ является рациональной функцией со знаменателем $1-t \operatorname{det} A$.

Кстати, аналогичными рассуждениями доказывается, что гомологическая $\zeta$-функция диффеоморфизма всегда рациональна (см. [15; с. 767-768]). К сожалению, фне везде определена (подошвы восходяших дисков в точности составляют множество, где $\phi$ не определена). ${ }^{4}$ Итерации $\phi$ только накапливают эту неопределенность. Однако, $в$ случае общего положения возможно построить некоторые объекты, которые можно подставить вместо $[p],[q]$ и $\phi_{*}$ в формулу $(1)$.

В этом месте я считаю уместньм предостеречь читателя, что вплоть до конца $\S 2$ изложение материала будет очень неформальньм. Предлагаемьй вниманию читателя текст можно рассматривать как программу, реализацией которой является работа [2]. Тем не менее я думаю, что понимание последуюшего абзаца весьма полезно для понимания [2] и настоящей статьи.

Начнем с "хорошего" клеточного разбиения $\partial_{1} W$. От "хорошего" разбиения мы потребуем, в частности, чтобы каждая клетка $e$ была представлена $C^{\infty}$-гладким вложением $\rho: D^{k} \rightarrow \partial_{1} W$ (где $k$ - размерность клетки). Естественно ожидать, что такое разбиение можно построить исходя из стратификации многообразия $\partial_{1} W$ устойчивыми многообразиями градиента некоторой функции Морса. Применив диффеоморфизм $\Phi^{-1}$, перенесем это разбиение на $\partial_{0} W$. Отображение $(-v)^{\rightsquigarrow}$ из $\partial_{1} W$ в $\partial_{0} W$ не является непрерьвным, но, тем не менее, можно попытаться так изменить $v$ посредством малого возмущения, чтобы отображение $(-w)^{\rightsquigarrow}$, соответствующее новому градиенту $w$, сохраняло клеточную структуру. Чтобы объяснить, почему это должно получаться, рассмотрим критическую точку $p$ функции $f$; пусть ind $p=k$. Положим $S_{-}(p, v)=D(p, v) \cap \partial_{0} W, S_{+}(p, v)=D(p,-v) \cap \partial_{1} W$. Изменяя в случае надобности $v$ посредством малого возмущения, можно предполагать, что все многообразия $S_{+}(p, v)$ трансверсальны ко всем клеткам разбиения $\partial_{1} W$. В частности, клетки $\partial_{1} W$ размерности $k$ будут пересекаться только с многообразиями $S_{+}(p, v)$, соответствуюшими критическим точкам $p$ индекса не более $k$. Пусть $e-$ клетка $\partial_{1} W$ размерности $k$.

\footnotetext{
${ }^{4}$ В [16] Новиков пишет: "В нашем случае мы имеем не отображение, а кобордизм с двумя равными границами, порождающий $\mathbb{Z}$ покрытие над компактным многообразием".
} 
Та часть $e$, где $(-v)^{\rightsquigarrow}$ не определено, является объединением множеств $S_{+}(p, v) \cap e$, где $p$ пробегает все критические точки $f$ индекса $\leqslant k$. Геометрическая картина поведения нисходяших траекторий такова. Градиентньй спуск стягивает множество $S_{+}(p, v) \cap e$ вниз к точке $p$ (за бесконечное время). Если мы попытаемся опуститься еше ниже к $\partial_{0} W$, то получим отображение, многозначное в том смысле, что каждой точке множества $S_{+}(p, v) \cap$ е соответствует множество $S_{-}(p, v)$. Заметим, однако, что $\operatorname{dim} S_{-}(p, v) \leqslant k-1$. Предположим теперь, что для любого $s$ все множества $S_{-}(p, v)$ при ind $p \leqslant s+1$ содержатся в $s$-остове $\partial_{0} W^{[s]}$ (что правдоподобно ввиду процедуры клеточной аппроксимации). В этом случае наше “отображение" $(-v)^{\rightsquigarrow}$ определит непрерывное отображение

$$
W_{k}: C_{k}^{(1)}=\partial_{1} W^{[k]} / \partial_{1} W^{[k-1]} \rightarrow C_{k}^{(0)}=\partial_{0} W^{[k]} / \partial_{0} W^{[k-1]}
$$

а также отображение $C_{k}^{(0)}$ в себя, поскольку $\Phi$ отождествляет $\partial_{0} W$ с $\partial_{1} W$. Конечно, пространство $C_{k}^{(1)}$ является букетом $k$-мерных сфер. Обозначим его гомологии $H_{k}$; это свободная абелева группа, и отображение $W_{k}$ индуцирует ее эндоморфизм. Пусть $r, q$-критические точки $f$, соответственно, индексов $k+1, k$. С помошњю тех же рассуждений, что и вьше, можно показать, что многообразие $S_{-}(r, v)$ определяет цикл пары $\left(\partial_{0} W^{[k]}, \partial_{0} W^{[k-1]}\right)$, т.е. элемент $[r] \in H_{k}\left(\partial_{0} W^{[k]}, \partial_{0} W^{[k-1]}\right)$. Индекс пересечения со сферой $S_{-}(q, v)$ определяет гомоморфизм $\lambda(q): H_{k} \rightarrow \mathbb{Z}$, и мы получаем: $n_{k}(r, q ; v)=\lambda(q)\left(\xi^{k}([r])\right)$. Теперь достаточно применить утверждение $(2)$, и мы видим, что новиковский коэффициент инцидентности $n(r, q ; v)$ является рациональной функцией.

Теперь я сделаю два замечания об изложенной программе.

1) Возмушения векторного поля $v$, о которых говорилось выше, могут быть выбраны $C^{0}$-мальми, но, вообше говоря, не $C^{\infty}$-малыми.

2) Мы сознательно упростили изложение, работая с клеточньми разбиениями многообразий $\partial_{1} W$ и $\partial_{0} W$. На самом деле это не приводит к цели; вместо клеточных разбиений нужно рассматривать разложения на ручки этих многообразий (множества $D_{\delta}($ ind $\leqslant k ; v)$ в обозначениях работы $\left.[2]\right)$.

\section{§. Краткий обзор результатов работы $[2]$}

Ниже мы будем использовать технику и результаты из [2], поэтому в этом параграфе мы приводим некоторые основные идеи и результаты работы [2].

3.1. $\delta$-тонкие разложения на ручки. В этом пункте $W$ - риманов кобордизм размерности $n, f: W \rightarrow[a, b]-$ функция Морса на $W$ и $v-f$-градиент.

Пусть $x \in W$ и $\delta>0$. Предположим, что для некоторого $\delta_{0}>\delta$ ограничение экспоненциального отображения $\exp _{q}: T_{q} W \rightarrow W$ на диск $B^{n}\left(0, \delta_{0}\right)$ есть диффеоморфизм на свой образ. Пусть $B_{\delta}(p)$ (соответственно, $\left.D_{\delta}(p)\right)$ обозначает риманов открытый шар (соответственно, замкнутый шар) радиуса $\delta$ с центром $p$. Мы будем использовать обозначения $B_{\delta}(p), D_{\delta}(p)$ только в случае, если $\delta$ удовлетворяет вьшеуказанным ограничениям. 
Положим

$$
\begin{gathered}
B_{\delta}(p, v)=\left\{x \in W \mid \exists t \geqslant 0: \gamma(x, t ; v) \subset B_{\delta}(p)\right\}, \\
D_{\delta}(p, v)=\left\{x \in W \mid \exists t \geqslant 0: \gamma(x, t ; v) \subset D_{\delta}(p)\right\}, \\
D(p, v)=\left\{x \in W \mid \lim _{t \rightarrow \infty} \gamma(x, t ; v)=p\right\} .
\end{gathered}
$$

Обозначим символом $D$ (ind $\leqslant s ; v)$ объединение множеств $D(p, v)$, где $p$ пробегает критические точки $f$ индекса $\leqslant s$. Символом $B_{\delta}($ ind $\leqslant s ; v)$ и $D_{\delta}($ ind $\leqslant s ; v)$ обозначим объединение множеств $B_{\delta}(p, v)$ и, соответственно, множеств $D_{\delta}(p, v)$, где $p$ пробегает критические точки $f$ индекса $\leqslant s$. Обозначения $D_{\delta}($ ind $=s ; v)$ или $B_{\delta}($ ind $\geqslant s ; v)$ ясны теперь без дополнительных объяснений. Объединение всех $D(p, v)$ обозначается символом $D(v)$, объединение всех $D_{\delta}(p, v)$ - символом $D_{\delta}(v)$. Примем также следующее соглашение: $D_{0}(p, v)=D(p, v), D_{0}(v)=D(v)$ и т. д.

Пусть $\phi: W \rightarrow[a, b]$ - упорядоченная функция Морса с упорядочивающей последовательностью $\left(a_{0}<a_{1}<\cdots<a_{n+1}\right)$. Пусть $w$ есть $\phi$-градиент. Положим $W_{i}=\phi^{-1}\left(\left[a_{i}, a_{i+1}\right]\right)$.

ОПРЕДЕЛЕНИЕ 3.1. Скажем, что $v$ является $\delta$-разделенным по отношению $\kappa \phi$ (и упорядочивающей последовательности $\left.\left(a_{0}, \ldots, a_{n+1}\right)\right)$, если

i) для каждого $i$ и каждого $p \in S_{i}(f)$ имеем $D_{\delta}(p) \subset W_{i}^{\circ}$;

ii) для каждого $i$ и каждого $p \in S_{i}(f)$ найдется функция Морса $\psi: W_{i} \rightarrow\left[a_{i}, a_{i+1}\right]$, согласованная с $\left(\phi \mid W_{i}, w\right)$, и регулярное значение $\lambda$ функции $\psi$ такие, что

$$
D_{\delta}(p) \subset \psi^{-1}(] a_{i}, \lambda[)
$$

и для каждого $q \in S_{i}(f), q \neq p$, имеем

$$
D_{\delta}(q) \subset \psi^{-1}(] \lambda, a_{i+1}[) .
$$

Мы говорим, что $v$ является $\delta$-разделеннылм, если он $\delta$-разделен по отношению к некоторой упорядоченной функции Морса $\phi: W \rightarrow[a, b]$, согласованной с $(f, v)$.

Ясно, что каждый $f$-градиент, удовлетворяющий условию почти-трансверсальности, является $\delta$-разделенным при некотором $\delta>0$.

ПРЕДЛОЖЕНИЕ 3.2. Пусть $v$ есть $\delta_{0}$-разделенный $f$-градиент. Тогда $\forall \delta \in$ $\left[0, \delta_{0}\right] u \forall s, 0 \leqslant s \leqslant n$ :

1. $D_{\delta}($ ind $\leqslant s ; v)$ компактно;

2. $\bigcap_{\theta>\delta} B_{\theta}($ ind $\leqslant s ; v)=D_{\delta}($ ind $\leqslant s ; v)$;

3. $е с л u \delta>0, m o \overline{B_{\delta}(\text { ind } \leqslant s ; v)}=D_{\delta}($ ind $\leqslant s ; v)$.

Итак, набор нисходящих дисков $D(p, v)$ образует стратифицированное многообразие, а $\delta$-утолщенные нисходящие диски $B_{\delta}(v)$ - окрестность этого многообразия. В предыдущем утверждении мы перечислили некоторые естественные свойства этих объектов. Для того чтобы изучить подробнее эти свойства, мы введем в следующем пункте новые понятия: $s$-подмногообразие и $t s$-подмногообразие.

3.2. $s$-подмногообразия и $t s$-подмногообразия. Пусть $\mathbb{A}=\left\{A_{0}, \ldots, A_{k}\right\}$-конечная последовательность подмножеств топологического пространства $X$. Положим $\mathbb{A}_{(\leqslant s)}=A_{0} \cup \cdots \cup A_{s}, \mathbb{A}_{(s)}=A_{s}$. Будем говорить, что $\mathbb{A}$ - компактное семейство, если $\mathbb{A}_{(\leqslant s)}$ компактно для каждого $s$. 
ОПРЕДЕЛЕНИЕ 3.3. Пусть $M$ - многообразие без края. Конечная последовательность $\mathbb{X}=\left\{X_{0}, \ldots, X_{k}\right\}$ подмножеств $M$ назьвается $s$-подмногообразием многообразия $M$, если

1. каждое $X_{i}$ является подмногообразием многообразия $M$ размерности $i$ с тривиальным нормальным расслоением;

2. $\mathbb{X}$ является компактным семейством;

3. для $i \neq j$ вьполнено $X_{i} \cap X_{j}=\varnothing$.

Пусть $\Phi: M \rightarrow N-$ диффеоморфизм и $\mathbb{X}-s$-подмногообразие в $M$. Символом $\Phi(\mathbb{X})$ мы обозначаем $s$-подмногообразие $N$, определенное формулой $\Phi(\mathbb{X})_{(i)}=\Phi\left(\mathbb{X}_{(i)}\right)$.

Пусть $\mathbb{X}, \mathbb{Y}$ - два $s$-подмногообразия $M$. Мы говорим, что $\mathbb{X}$ трансверсально $\kappa \mathbb{Y}$ (пишем: $\mathbb{X} \pitchfork \mathbb{Y}$ ), если $\mathbb{X}_{(i)} \pitchfork \mathbb{Y}_{(j)}$ для всех $i, j ;$ мы говорим, что $\mathbb{X}$ почти трансверсально $\kappa \mathbb{Y}($ (пшшем: $\mathbb{X} \nmid \mathbb{Y})$, если $\mathbb{X}_{(i)} \pitchfork \mathbb{Y}(j)$ для $i+j<\operatorname{dim} M$. Заметим, что условие $\mathbb{X} \nmid \mathbb{Y}$ эквивалентно следуюшему: $\mathbb{X}_{(\leqslant i)} \cap \mathbb{Y}_{(\leqslant j)}=\varnothing$ при $i+j<\operatorname{dim} M$.

Для $s$-подмногообразий вьполнено следующее утверждение, являющееся аналогом теоремы Тома о трансверсальности: если $\mathbb{X}, \mathbb{Y}-s$-подмногообразия $M$, то найдется достаточно малая изотопия $\Phi_{t}$ многообразия $M$ такая, что $\Phi_{1}(\mathbb{X}) \nmid \mathbb{Y}($ см. [2; теорема 2.3]).

ОПРЕДЕЛЕНИЕ 3.4 . Пусть $X$ - топологическое пространство, $\mathbb{A}=\left\{A_{0}, \ldots, A_{k}\right\}-$ компактное семейство подмножеств $X, I$ - открытый интервал $] 0, \delta_{0}[$. Хорошей фундаментальной системой окрестностей семейства $\mathbb{A}$ (сокрашение: $g \mathrm{fn} n$-системой для $\mathbb{A}$ ) называется семейство $\mathbf{A}=\left\{A_{s}(\delta)\right\}_{\delta \in I, 0 \leqslant s \leqslant k}$ открытых подмножеств $X$, удовлетворяющее следуюшим условиям:

1. $\underline{A_{s} \subset A_{s}}\left(\delta_{1}\right) \subset A_{s}\left(\delta_{2}\right)$ для произвольных $s$ и для $\delta_{1}<\delta_{2}$;

2. $\overline{A_{\leqslant j}(\delta)}=\bigcap_{\theta>\delta}\left(A_{\leqslant j}(\theta)\right)$ для произвольных $\delta$ и $j$;

3. $A_{(\leqslant j)}=\bigcap_{\theta>0}\left(\mathbf{A}_{(\leqslant j)}(\theta)\right)$ для произвольного $j$.

$I$ назьвается интервалом определения системы. $\mathbb{A}$ назьвается ядром $\mathbf{A}$, и $\mathbf{A}$ называется утолщением $\mathbb{A}$. Мы будем обозначать $A_{s}(\delta)$ также символом $\mathbf{A}_{(s)}(\delta)$ и $A_{\leqslant i}(\delta)$ - символом $\mathbf{A}_{(\leqslant i)}(\delta)$. Иногда мы будем писать $\mathbf{A}_{s}(0)$ вместо $A_{s}$.

Мы выбрали название "хорошая фундаментальная система" по следуюшей причине: если $X$ компактно, то для произвольных $\delta \geqslant 0$ и $s$ семейство $\left\{A_{\leqslant s}(\theta)\right\}_{\theta>\delta}$ является фундаментальной системой окрестностей $\overline{\mathbf{A}_{(\leqslant s)}(\delta)}$ (это легко следует из условий 1-3).

Если $M$ - многообразие без края, $\mathbb{X}-s$-подмногообразие $M$ и $\mathbf{X}-g f n$-система для $\mathbb{X}$, то $\mathbf{X}$ назьвается $t s$-подмногообразием $c$ ядром $\mathbb{X}$. Также мы говорим, что $\mathbf{X}$ является утолщением $\mathbb{X}$. Пусть $\mathbf{X}=\left\{X_{s}(\delta)\right\}_{\delta \in I, 0 \leqslant s \leqslant k}-t s$-подмногообразие. Мы будем обозначать $X_{i}(\delta)$ символом $\mathbf{X}_{(i)}(\delta)$ и $X_{\leqslant i}(\delta)$ - символом $\mathbf{X}_{(\leqslant i)}(\delta)$.

Приведем важньй пример $g f n$-системы. Пусть $f: W \rightarrow[a, b]$ - функция Морса на кобордизме $W$, и пусть $v-f$-градиент. Предположим, что $v$ является $\varepsilon$-разделеннгм. Тогда семейство $\left\{B_{\delta}(\text { ind }=s ; v)\right\}_{\delta \in] 0, \epsilon[, 0 \leqslant s \leqslant n}$ является $g f n$-системой для $\mathbb{D}(v)$. Если $W$ - замкнутое многообразие, то это семейство является $t s$-подмногообразием с ядром $\mathbb{D}(v)$. Эта $g f n$-система будет обозначаться $\mathbf{D}(v)$. (Если $\partial W \neq \varnothing$, то $\mathbb{D}(v)$ - семейство многообразий с краем, но ни использовать, ни формулировать соответствуюшее обобщение понятия $t s$-подмногообразия мы не будем.) 
Пусть $f: W \rightarrow[a, b]$ - функция Морса на кобордизме $W$ и $v-f$-градиент. Пусть $X \subset \partial_{1} W$. Множество $T(X, v)$, образованное всеми $(-v)$-траекториями, начинаюшимися в $X$, будет назьваться следом $X$. Множество $T(X, v)$ не обязано быть компактным, даже если $X$ компактно. Однако можно показать, что если $X$ компактно, то $T(X, v) \cup D_{\delta}(v)$ компактно при каждом $\delta$ (см. [2; лемма 2.7]).

Теперь мы определим понятие следа для $s$-подмногообразий. Пусть $\mathbb{A}$ есть $s$-подмногообразие в $\partial_{1} W$. Компонента размерности $k$ семейства $\mathbb{T}(\mathbb{A}, v)$ должна состоять из следа $\mathbb{T}\left(A_{k-1}, v\right)$ и нисходяших дисков $D(p, v)$ критических точек $p$ c ind $p \leqslant k$.

Перейдем к точным определениям. С этого места и до конца параграффа мы предполагаем, что $v$ удовлетворяет условию почти-трансверсальности и что $\mathbb{A} \nmid \mathbb{D}_{b}(-v)$.

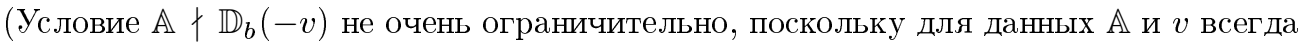
можно выбрать $C^{\infty}$-малое возмущение $v$ такое, что это условие будет выполнено, см. [2; лемма 4.2].)

ОПРЕДЕЛЕНИЕ 3.5. ПоЛОЖим $T A_{i}(v)=T\left(A_{i-1}, v\right) \cup D($ ind $=i ; v)\left(A_{-1}=\varnothing\right.$ по определению). Символом $\mathbb{T}(\mathbb{A}, v)$ обозначим семейство $\left\{T A_{i}(v)\right\}_{0 \leqslant i \leqslant k+1}$ подмножеств $W$ и символом $(-v)_{[b, \lambda]}^{\rightsquigarrow}(\mathbb{A})-$ семейство $\left\{T A_{i+1}(v) \cap f^{-1}(\lambda)\right\}_{0 \leqslant i \leqslant k}$ подмножеств $f^{-1}(\lambda)$. Если значения $b, \lambda$ ясны из контекста, то мы будем писать $(-v)^{\rightsquigarrow}(\mathbb{A})$ вместо $(-v)_{[b, \lambda]}^{\rightsquigarrow}(\mathbb{A})$. Семейство $\mathbb{T}(\mathbb{A}, v)$ будет назьваться следом $\mathbb{A}$, а семейство $(-v)_{[b, \lambda]}^{\rightsquigarrow}(\mathbb{A})$ $-(-v)^{\rightsquigarrow}$-образом $\mathbb{A}$.

Можно показать, что $\mathbb{T}(\mathbb{A}, v)$ и $(-v)_{[b, \lambda]}^{\rightsquigarrow}(\mathbb{A})$ являются компактными семействами. Далее, если $\lambda$ - регулярное значение $f$, то $(-v)_{[b, \lambda]}^{\rightsquigarrow}(\mathbb{A})$ есть $s$-подмногообразие $f^{-1}(\lambda)$ (см. [2; лемма 2.9]).

Теперь мы можем определить понятие следа для $t s$-подмногообразий. Пусть $\mathbf{A}-$ $t s$-подмногообразие $\partial_{0} W$ с ядром $\mathbb{A}$. Нижеследующие определение и предложение позволяют ввести $g f n$-систему с ядром $\mathbb{T}(\mathbb{A}, v)$, которая и будет назьваться следом $\mathbf{A}$.

ОПРЕДЕЛЕНИЕ 3.6. ПУсть $\mathbf{A}=\left\{A_{s}(\delta)\right\}_{\delta \in] 0, \delta_{0}[, 0 \leqslant s \leqslant k}-t s$-подмногообразие $\partial_{1} W$ с ядром $\mathbb{A}$. Предположим, что $v-\delta_{1}$-разделен. Для $0<\delta<\min \left(\delta_{0}, \delta_{1}\right)$ положим $T A_{s}(\delta, v)=T\left(A_{s-1}(\delta), v\right) \cup B_{\delta}($ ind $=s ; v)\left(A_{-1}(\delta)=\varnothing\right.$ по определению).

ПРЕДЛОЖЕНИЕ 3.7 ([2; предложение 2.12]). Существует $\epsilon \in] 0, \min \left(\delta_{0}, \delta_{1}\right)[$ ma-

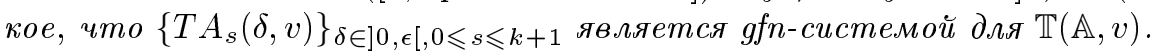

$g f n$-система, введенная в предложении 3.7 , будет обозначаться $\mathbf{T}(\mathbf{A}, v)$ и называться следом $\mathbf{A}$.

3.3. Быстрые потоки. Пусть $M$ - замкнутое многообразие размерности $m, f-$ функция Морса на $M$ и $v-f$-градиент, удовлетворяюший условию почти-трансверсальности. Пусть $k$ - целое число, $U$ - открытая окрестность множества $D($ ind $\leqslant k ;-v)$ и $V$ - открытая окрестность множества $D($ ind $\leqslant m-k-1 ; v)$. Ясно, что порожденный полем $v$ поток $\Phi_{t}$ снесет $M \backslash V$ на $U$ через достаточно большое время. (А именно, обозначим диффеоморфизм $t \mapsto \gamma(x, t ; v)$ многообразия $M$ посредством $\Phi(v, t)$. Тогда для достаточно большого $T$ мы имеем: $\Phi(v, T)(M \backslash V) \subset U$.$) Если$ поток $\Phi_{t}$ вьполняет эту операцию за малое время и, более того, если $v$ имеет малую норму, то мы говорим, что $\Phi_{t}$ быстрый. Ниже дано точное определение. 
ОПРЕДЕЛЕНИЕ 3.8. Пусть $v-f$-градиент, удовлетворяюший условию почти-трансверсальности, и пусть $\epsilon>0, t \geqslant 0$. Мы говорим, что поток, порожденный векторным полем $v$, является $(t, \epsilon)$-бьстрыцм, если для каждого $s, 0 \leqslant s \leqslant m$, вьполнено:

$$
\begin{aligned}
\Phi(v, t)\left(M \backslash B_{\epsilon}(\mathrm{ind} \leqslant s ; v)\right) & \subset B_{\epsilon}(\text { ind } \leqslant m-1-s ;-v), \\
\Phi(-v, t)\left(M \backslash B_{\epsilon}(\text { ind } \leqslant s ;-v)\right) & \subset B_{\epsilon}(\text { ind } \leqslant m-1-s ; v) .
\end{aligned}
$$

(Для краткости мы иногда будем говорить, что сам градиент $v$ является $(t, \epsilon)$-быстрым.)

Несложно проверить, что каждьй поток $v$, удовлетворяющий условию почти-трансверсальности, является $(t, \epsilon)$-быстрым при некоторых $t, \epsilon$.

Замечание о терминологии. Определение быстрого потока, принятое в настоящей работе, отличается от определения быстрого потока из работы [2]: это разные понятия! Мы не будем использовать в настоящей работе понятие быстрого потока в смысле [2], так что не может возникнуть никаких недоразумений. Термин "быстрый поток" в смысле определения 3.8 мы переводим на английский язык термином "rapid flow", а термин "быстрьй поток" в смысле работы [2] - термином "quick flow". С быстрыми потоками в смысле настоящей работы удобнее работать, но теорему об их сушествовании удобней доказывать, используя быстрые потоки в смысле [2] .

ОПРЕДЕЛЕНИЕ 3.9. Пусть $f: W \rightarrow[a, b]$ - функция Морса на кобордизме, и пусть $u, v-f$-градиенты. Мы говорим, что $u$ и $v$ әквивалентны, если $u(x)=\phi(x) v(x)$, где $\phi: W \rightarrow \mathbb{R}-C^{\infty}$-гладкая функция такая, что $\phi(x)>0$ для всех $x$ и $\phi(x)=1$ для $x$ из окрестности множества $S(f)$.

ТЕОРема 3.10. Пусть $M$ - замкнутое риманово многообразие и пусть $C>0$, $t>0$.

Существуют функиия Морса $f: M \rightarrow \mathbb{R}$ u $f$-градиент $v$, удовлетворяющий условию почти-трансверсальности, такие, что для каждого $\epsilon>0$ найдется $(t, \epsilon)$-бъстрый $f$-градиент $u$, әквивалентный $v$ и удовлетворяющий $\|u\| \leqslant C$.

Эта теорема была доказана в [2; следствие 1.14].

3.4. Условие (RP). В этом пункте $W$ - риманов кобордизм, $f: W \rightarrow[a, b]-$ функция Морса, $v-f$-градиент, удовлетворяющий условию почти-трансверсальности, $n=\operatorname{dim} W$.

ОПРЕДЕЛЕНИЕ 3.11. Мы говорим, что $v$ имеет $R$-пару (или, эквивалентно, что $v$ удовлетворяет условию (RP)), если найдутся функции $\phi_{0}: \partial_{0} W \rightarrow \mathbb{R}, \phi_{1}: \partial_{1} W \rightarrow \mathbb{R}$, и их градиенты $u_{0}$ и, соответственно, $u_{1}$, удовлетворяющие условию почти-трансверсальности, и число $\delta>0$ такие, что для каждого $s$ выполняется следующее условие:

$$
\delta \text { - интервал определения семейств } \mathbf{T}\left(\mathbf{D}\left(u_{1}\right), v\right) \text { и } \mathbf{T}\left(\mathbf{D}\left(-u_{0}\right),-v\right),
$$

$$
\begin{gathered}
\text { градиенты } v, u_{0} \text { и } u_{1} \text { являются } \delta \text {-разделенньми, } \\
\frac{\mathbf{T}\left(\mathbf{D}\left(u_{1}\right), v\right)_{(\leqslant s+1)}(\delta)}{\mathbf{T}\left(\mathbf{D}\left(-u_{0}\right),-v\right)_{(\leqslant s+1)}(\delta)} \cap \partial_{0} W \subset \mathbf{D}\left(u_{0}\right)_{(\leqslant s)}(\delta), \\
\partial_{1} W \subset \mathbf{D}\left(-u_{1}\right)_{(\leqslant s)}(\delta) .
\end{gathered}
$$

Множество $f$-градиентов $v$, удовлетворяющих (RP), будет обозначаться символом $\mathscr{G} \mathscr{R} \mathscr{P}(f)$. 
Tеорема 3.12. Множество $\mathscr{G} \mathscr{R} \mathscr{P}(f)$ является $C^{0}$-плотным в $\mathscr{G}(f)$.

ДоказАтельство. Пусть $v \in \mathscr{G} \mathscr{A}(f)$, и пусть $\phi_{1}: \partial_{1} W \rightarrow \mathbb{R}, \phi_{0}: \partial_{0} W \rightarrow \mathbb{R}-$ функции Морса, $u_{1}, u_{0}$ - их градиенты, удовлетворяющие условию почти-трансверсальности. План доказательства состоит в следующем. Чтобы получить $f$-градиент $w \in \mathscr{G} \mathscr{R} \mathscr{P}(f)$, мы изменим $v$, добавив горизонтальные компоненты: $\xi_{1}$ около $\partial_{1} W$ и $\xi_{0}$ около $\partial_{0} W$. Сначала мы проведем это построение, не налагая никаких условий на $u_{1}, u_{0}$, и получим новый $f$-градиент $w$, удовлетворяющий $(\mathrm{RP})$. Далее мы покажем, как выбрать $u_{1}, u_{0}$, чтобы $w$ был $C^{0}$-близок к $v$.

Перейдем к доказательству. При необходимости возмущая $v$, можно предположить, что $(-v)^{\rightsquigarrow}\left(\mathbb{D}\left(u_{1}\right)\right)$ почти трансверсален к $\mathbb{D}\left(-u_{0}\right)$, т.е. $(-v)^{\rightsquigarrow}\left(\mathbb{D}_{(\leqslant k)}\left(u_{1}\right)\right)$ не пересекает $\mathbb{D}_{(\leqslant m)}\left(-u_{0}\right)$, если $k+m<n-1$. Поэтому для достаточно малого $\delta$ выполнено

$$
(-v)^{\rightsquigarrow}\left(D_{\delta}\left(\text { ind } \leqslant k ; u_{1}\right)\right) \cap D_{\delta}\left(\text { ind } \leqslant m ;-u_{0}\right)=\varnothing, \text { если } k+m<n-1,
$$

(это вытекает из свойств $g f n$-систем).

Для краткости обозначений положим

$$
\begin{array}{ll}
A_{k}^{-}(\delta)=D_{\delta}\left(\text { ind } \leqslant k ; u_{1}\right), & A_{k}^{+}(\delta)=D_{\delta}\left(\text { ind } \leqslant k ;-u_{1}\right) \\
B_{k}^{-}(\delta)=D_{\delta}\left(\text { ind } \leqslant k ; u_{0}\right), & B_{k}^{+}(\delta)=D_{\delta}\left(\text { ind } \leqslant k ;-u_{0}\right) .
\end{array}
$$

(Итак, $A_{k}^{-}(\delta)$ - " $\delta$-утолшение $k$-остова $\partial_{1} W$ ", и $A_{k}^{+}(\delta)$ - " $\delta$-утолшение $k$-остова дуального клеточного разбиения $\partial_{1} W$ ". Аналогичным образом можно интерпретировать и множества $B_{k}(\delta)^{ \pm}$.)

Пусть $\xi_{0}-\phi_{0}$-градиент, эквивалентный $u_{0}$, и пусть $\xi_{1}-\phi_{1}$-градиент, эквивалентный $u_{1}$. При достаточно большом $T>0$ и произвольном $k$ имеем:

$$
\begin{aligned}
& \Phi\left( \pm \xi_{0}, T\right)\left(\partial_{0} W \backslash B_{k}^{\mp}(\delta)\right) \subset B_{n-k-2}^{ \pm}(\delta), \\
& \Phi\left( \pm \xi_{1}, T\right)\left(\partial_{1} W \backslash A_{k}^{\mp}(\delta)\right) \subset A_{n-2-k}^{ \pm}(\delta) .
\end{aligned}
$$

Положим $\Phi_{0}=\Phi\left(\xi_{0}, T\right), \Phi_{1}=\Phi\left(\xi_{1}, T\right)$ и добавим к $v$ две горизонтальные компоненты: $\xi_{0}$ около $\partial_{0} W$ и $\xi_{1}$ около $\partial_{1} W$ (см. п. 2.1). Если мы выберем вспомогательную функцию $h$, удовлетворяющую $\int_{0}^{l} h(\tau) d \tau=T$, то тогда для результирующего векторного поля $v^{\prime}$ получим:

$$
\left(-v^{\prime}\right)^{\rightsquigarrow}=\Phi_{0} \circ(-v)^{\rightsquigarrow} \circ \Phi_{1}^{-1} .
$$

Теперь ясно, что для $v^{\prime}$ выполнены условия (RP3) и (RP4). В самом деле, множество $A_{m}^{-}(\delta)$ переводится в себя отображением $\Phi_{1}^{-1}$, результат переводится в $\partial_{0} W \backslash$ $B_{n-2-m}^{+}(\delta)$ посредством $(-v)^{\rightsquigarrow}$, и $\Phi_{0}$ переводит $\partial_{0} W \backslash B_{n-2-m}^{+}(\delta)$ в $B_{m}^{-}(\delta)$. Это доказывает (RP3); условие (RP4) доказьвается аналогично. (Конечно, нужно выбирать $\delta$ достаточно малым, чтобы удовлетворить (RP1) и (RP2). Нужно также позаботиться о том, чтобы $v^{\prime}$ был $f$-градиентом. Детали см. в [2; $\S 4$.) Теперь мы объясним, как сделать норму $\left\|v^{\prime}-v\right\|$ малой. Если мы выберем $u_{0}, u_{1}$ так, чтобы удовлетворить 
заключениям теоремы о быстрых потоках, мы получим векторные поля $\xi_{0}, \xi_{1}$ с малой нормой, для которых свойства $(5),(6)$ уже выполнены для достаточно малого $T$. Следовательно, измененное векторное поле $v^{\prime}$ будет $C^{0}$-близко к $v$.

3.5. $R$-системы и гомологический градиентный спуск (первый вариант). Как мы уже отмечали, отображение $(-v)^{\rightsquigarrow}$ определено не везде. Тем не менее, иногда возможно определить аналог “гомоморфизма, индуцированного $(-v)^{\rightsquigarrow}$ в гомологиях". Более точно, в некоторых случаях (например, когда $v$ удовлетворяет (RP)) можно определить гомоморфизм $H(v): H_{*}\left(\partial_{1} W \backslash B_{1}, A_{1}\right) \rightarrow H_{*}\left(\partial_{0} W \backslash B_{0}, A_{0}\right)$, где $A_{i}, B_{i}$ - некоторые непересекающиеся подмножества $\partial_{i} W$. Построение такого гомоморфизма $H(v)$ осушествлено в $[2 ; \S 4]$. Сейчас мы вкратце опишем его. При этом мы хотели бы подчеркнуть, что этот гомоморфизм не индуцирован непрерывным отображением пар.

ОПРЕДЕЛЕНИЕ 3.13. Пусть $\Lambda=\left\{\lambda_{0}, \ldots, \lambda_{k}\right\}$ - конечное множество регулярных значений $f$ такое, что $\lambda_{0}=a, \lambda_{k}=b$, для каждого $i$ мы имеем $\lambda_{i}<\lambda_{i+1}$ и на отрезке $\left[\lambda_{i}, \lambda_{i+1}\right]$ есть в точности одно критическое значение $f$. Значения $\lambda_{i}, \lambda_{i+1}$ называются смежснылми. Множество пар $\left\{\left(A_{\lambda}, B_{\lambda}\right)\right\}_{\lambda \in \Lambda}$ называется $R$-системой для $(f, v)$, если вьполнены следующие условия.

(RS1) Для каждого $\lambda \in \Lambda$ множества $A_{\lambda}$ и $B_{\lambda}$ суть непересекающиеся компактные подмножества в $f^{-1}(\lambda)$.

(RS2) Пусть $\lambda, \mu \in \Lambda$-смежные. Тогда для каждого $p \in S(f) \cap f^{-1}([\lambda, \mu])$ вьполнено или

i) $D(p, v) \cap f^{-1}(\lambda) \subset \operatorname{Int} A_{\lambda}$, или

ii) $D(p,-v) \cap f^{-1}(\mu) \subset \operatorname{Int} B_{\mu}$

(RS3) Пусть $\lambda, \mu \in \Lambda$ - смежные. Тогда $(-v)_{[\mu, \lambda]}^{\varkappa}\left(A_{\mu}\right) \subset \operatorname{Int} A_{\lambda}$ и $v_{[\lambda, \mu]}^{\rightsquigarrow 2}\left(B_{\lambda}\right) \subset \operatorname{Int} B_{\mu}$.

$R$-системы обладают следуюшими свойствами (см. [2; $\$ 4.3])$.

(1) Если $\left\{\left(A_{\lambda}, B_{\lambda}\right)\right\}_{\lambda \in \Lambda}-R$-система для $(f, v)$, то для каждого $f$-градиента $w$, достаточно $C^{0}$-близкого к $v,\left\{\left(A_{\lambda}, B_{\lambda}\right)\right\}_{\lambda \in \Lambda}$ является $R$-системой для $(f, w)$.

(2) Пусть $N$ - подмногообразие $\partial_{1} W \backslash B_{b}$ такое, что $N \backslash \operatorname{Int} A_{b}$ компактно. Тогда $N^{\prime}=v_{[b, a]}^{\rightsquigarrow}(N)$ - подмногообразие $\partial_{0} W \backslash B_{a}$ такое, что $N^{\prime} \backslash \operatorname{Int} A_{a}$ компактно.

(3) Существует гомоморфизм $H(v): H_{*}\left(\partial_{1} W \backslash B_{b}, A_{b}\right) \rightarrow H_{*}\left(\partial_{0} W \backslash B_{a}, A_{a}\right)$ такой, что

(a) если $N$ - ориентированное подмногообразие $\partial_{1} W$, удовлетворяющее условию из п. (2), то $H(v)([N])=\left[v_{[b, a]}^{\rightsquigarrow}(N)\right]$;

(b) существует $\epsilon>0$ такое, что для каждого $f$-градиента $w$, удовлетворяющего $\|w-v\|<\epsilon$, выполнено $H(v)=H(w)$.

Здесь мы объясним основную идею конструкции $H(v)$ в следуюшем простом случае: мы предположим, что $f$ обладает единственной критической точкой $p$ и $D(p, v) \cap$ $\partial_{0} W \subset \operatorname{Int} A_{a}$. Более того, мы предположим, что $B_{a}=\varnothing, B_{b}=\varnothing$. Пусть $U$ - достаточно малая компактная окрестность $D(p,-v) \cap \partial_{1} W$ такая, что $(-v)^{\rightsquigarrow}(U) \subset \operatorname{Int} A_{a}$. Пусть $x \in H_{*}\left(\partial_{1} W \backslash B_{b}, A_{b}\right)$. Рассмотрим образ элемента $x$ в группе $H_{*}\left(\partial_{1} W, A_{1}\right)$. Приведя его по модулю $A_{b} \cup U$, получим элемент $x^{\prime} \in H_{*}\left(\partial_{1} W, A_{b} \cup U\right)$. С помощью аксиомы вырезания получим элемент $\bar{x} \in H_{*}\left(\partial_{1} W \backslash D(p,-v), A_{b} \cup U \backslash D(p,-v)\right)$. 
Любая сингулярная цепь, представляющая $\bar{x}$, лежит в области определения $(-v)^{\rightsquigarrow}$. Применим $(-v)_{*}^{\rightsquigarrow}$ и получим элемент в $H_{*}\left(\partial_{0} W, A_{0}\right)$, которьй, по определению, и есть $H(v)(x)$. (В приведенном выше определении читателю будет нетрудно узнать основную идею конструкции гомологического градиентного спуска, см. п. 2.5.)

\section{$\S$ 4. Условие $(\mathfrak{C})$ и оператор гомологического градиентного спуска}

Нам потребуется еще несколько определений, их мы введем в п. 4.1. Во второй части этого пункта мы напомним классическую комплекса Морса. Содержание этого пункта не является, строго говоря, необходимым для понимания остальной части $\S 4$, но с точки зрения логики изложения естественно начать именно с понятий, обсуждаемых в п. 4.1 .

В п. 4.2 мы вводим условие (ㄷ). Это условие аналогично условию (RP), но обладает некоторыми техническими преимуществами по сравнению c $(\mathrm{RP})$. B частности, множество всех $f$-градиентов, удовлетворяюших $(\mathfrak{C})$, является $C^{0}$-открытым в множестве всех градиентов. В п. 4.3 содержится еще один вариант конструкции оператора гомологического градиентного спуска.

Обозначения: в оставшейся части $\S 4 W$ - риманов кобордизм размерности $n$.

4.1. $H$-фильтрации кобордизмов и морсовские комплексы. В этом пункте $\phi: W \rightarrow[a, b]-$ упорядоченная функция Морса с упорядочивающей последовательностью $a_{0}=a<\cdots<a_{n+1}=b$ и $v-\phi$-градиент. Предположим, что $v$ является $\delta$-разделенньм. Пусть $\nu \in] 0, \delta]$ и $s$ - целое число между 0 и $n+1$. Положим

$$
\begin{gathered}
W^{\{\leqslant s\}}=\phi^{-1}\left(\left[a_{0}, a_{s+1}\right]\right), \quad W^{\{\geqslant s\}}=\phi^{-1}\left(\left[a_{s}, a_{n+1}\right]\right), \\
W^{[\leqslant s]}(\nu)=D_{\nu}(\text { ind } \leqslant s ; v) \cup \partial_{0} W \\
W^{\lceil s\rceil}(\nu)=\phi^{-1}\left(\left[a_{0}, a_{s}\right]\right) \cup D_{\nu}(\text { ind }=s ; v) .
\end{gathered}
$$

Мы будем назьвать все эти фильтрации " $H$-фильтрациями".

Приведенные вьше определения имеют смысл, разумеется, и в большей обшности, но только наложенные выше ограничения на $\phi$ и $v$ обеспечивают хорошие гомологические свойства фильтраций. Эти свойства представлены в следующем предложении. Для критической точки $p \in S_{k}(\phi)$ символом $d(p)$ мы обозначаем вложенный диск $D(p, v) \cap \phi^{-1}\left(\left[a_{k}, a_{k+1}\right]\right)$. В каждой критической точке $p \in S(\phi)$ выберем ориентацию нисходящего диска $D(p, v)$. Пусть $[p]$ обозначает образ в группе $H_{k}\left(W^{\{\leqslant k\}}, W^{\{\leqslant k-1\}}\right)$ фундаментального класса пары $(d(p), \partial d(p))$.

ПРЕДЛОЖЕНИЕ 4.1. Пусть $s \in \mathbb{N} u \nu \in] 0, \delta]$.

1. Включения $W^{[\leqslant s]}(\nu) \subset W^{\lceil s\rceil}(\nu) \subset W^{\{\leqslant s\}}$ являются гомотопическими әквивалентностями.

2. Включения пар

$$
\left(W^{[\leqslant s]}(\nu), W^{[\leqslant s-1]}(\nu)\right) \subset\left(W^{\lceil s\rceil}, W^{\{\leqslant s-1\}}\right) \subset\left(W^{\{\leqslant s\}}, W^{\{\leqslant s-1\}}\right)
$$

являются гомотопическими эквивалентностями.

3. $H_{*}\left(W^{\{\leqslant s\}}, W^{\{\leqslant s-1\}}\right)=0$, еслu* $\neq$ s. Групnа $H_{s}\left(W^{\{\leqslant s\}}, W^{\{\leqslant s-1\}}\right)-$ свободная абелева группа, порожсденная әлементами $[p], p \in S_{s}(\phi)$. 
Обозначим $H_{s}\left(W^{\{\leqslant s\}}, W^{\{\leqslant s-1\}}\right)$ символом $C_{s}$. Граничньй оператор точной последовательности тройки $\left(W^{\{\leqslant s\}}, W^{\{\leqslant s-1\}}, W^{\{\leqslant s-2\}}\right)$ определяет гомоморфизм $\partial_{s}: C_{s} \rightarrow C_{s-1}$; очевидно, мы имеем $\partial_{s} \circ \partial_{s+1}=0$ для всех $s$. Таким образом, градуированная группа $C_{*}$, вместе с граничным оператором $\partial_{*}$, образуют свободньй цепной комплекс. В том случае, когда $v$ удовлетворяет условию трансверсальности, можно дать другую конструкцию этого комплекса, которую обычно и назьвают комплексом Морса.

Пусть $k$ - натуральное число, $p \in S_{k}(\phi), q \in S_{k-1}(\phi)$. Символом $\Gamma(p, q ; v)$ обозначим множество интегральных кривых поля $(-v)$, соединяюших $p$ с $q$, причем каждая интегральная кривая рассматривается с точностью до замены параметра. Из условия трансверсальности несложно вывести, что $\Gamma(p, q ; v)$ - конечное множество. Выбранные ориентации нисходяших дисков позволяют приписать каждому $\gamma \in \Gamma(p, q ; v)$ знак $\varepsilon(\gamma) \in\{-1,1\}$

Теперь определим $C_{k}(v)$ как свободную абелеву группу, свободно порожденную критическими точками $\phi$ индекса $k$. Положим $n(p, q ; v)=\sum_{\gamma \in \Gamma(p, q ; v)} \varepsilon(\gamma)$ и определим гомоморфизм $\partial_{k}^{\prime}(v): C_{k}(v) \rightarrow C_{k-1}(v)$ формулой

$$
\partial_{k}^{\prime}(v)(p)=\sum_{q \in S_{k-1}(\phi)} n(p, q ; v) q
$$

Оказывается, что гомоморфизм $J_{k}: C_{k}(v) \rightarrow C_{k}$, переводяший $p$ в $[p]$, удовлетворяет $J_{k} \circ \partial_{k+1}^{\prime}(v)=\partial_{k+1} \circ J_{k+1} ;$ итак, $\left(C_{*}(v), \partial_{k}(v)\right)$ - цепной комплекс, изоморфньй $C_{*}$.

Чтобы определить “эквивариантный комплекс Морса”, рассмотрим регулярное накрытие $\mathscr{P}: W \rightarrow W$ со структурной группой $G$. Прообраз подмножества $A \subset W$ в $\widetilde{W}$ будет обозначаться посредством $\widetilde{A}$. В дополнение к ориентациям нисходящих дисков, для каждой точки $p \in S(\phi)$ выберем ее поднятие в $\widetilde{W}$, т.е. точку $\tilde{p}$ такую, что $\mathscr{P}(\tilde{p})=p$. Тогда определено поднятие диска $d(p)$ в $\widetilde{W}$. Символом $[\tilde{p}]$ обозначим образ в групше $H_{k}\left(\widetilde{W}^{\{\leqslant k\}}, \widetilde{W}^{\{\leqslant k-1\}}\right)$ фундаментального класса пары $(d(\tilde{p}), \partial d(\tilde{p}))$.

ПРЕДЛОЖЕНИЕ 4.2. $H_{*}\left(\widetilde{W}^{\{\leqslant s\}}, \widetilde{W}^{\{\leqslant s-1\}}\right)=0$, ecлu $* \neq s$.

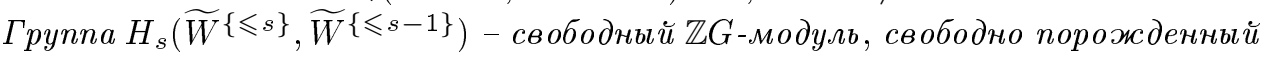
әлементами $[\tilde{p}], p \in S_{s}(\phi)$.

Аналогично указанному вьше способу, можно построить цепной комплекс $\left(\widetilde{C}_{*}(v), \widetilde{\partial}_{*}(v)\right)$ свободных $\mathbb{Z} G$-модулей, свободно порожденный в размерности $p$ критическими точками $\phi$ индекса $p$; и тогда граничный оператор определяется путем подсчета $v$-траекторий, соединяющих критические точки. Читатель может ознакомиться с деталями и доказательствами этих утверждений в [3; приложение].

4.2. Условие $(\mathfrak{C})$. Обозначения. Пусть $g: W \rightarrow[a, b]$ - функция Морса на кобордизме $W$ размерности $n, v-g$-градиент.

ОПрЕДЕЛЕНИЕ 4.3. Пусть $\lambda \in[a, b]$ - регулярное значение $f$, и пусть $A \subset \partial_{1} W$. Мы говорим, что $v$ cnyскает $A$ до $f^{-1}(\lambda)$, если каждая $(-v)$-траектория, начинающаяся в $A$, достигает $f^{-1}(\lambda)$. Пусть $X \subset W$. Мы говорим, что $v$ cnyскает $A$ до $f^{-1}(\lambda)$ без пересечения $c X$, если $v$ спускает $A$ до $f^{-1}(\lambda)$ и $T(A, v) \cap X=\varnothing$.

Понятия " $v$ поднимает $B$ до $f^{-1}(\lambda)$ " и " $v$ поднимает $B$ до $f^{-1}(\lambda)$ без пересечения с $Y$ " (где $B \subset \partial_{0} W, Y \subset W$ ) определяются аналогично. 
Напомним, что в п. 3.1 мы определили множества $B_{\delta}(\operatorname{ind} \leqslant s ; v)$ и $D_{\delta}($ ind $\leqslant s ; v)$. Введем еще одно множество со сходньми свойствами: положим $C_{\delta}($ ind $\leqslant s ; v)=$ $W \backslash B_{\delta}($ ind $\geqslant n-s-1 ; v)$. Следуюшая лемма доказывается стандартными рассуждениями из теории Морса.

ЛЕмма 4.4. Предположим, что $v$ является $\delta$-разделенным по отношению $к$ $\phi$ и упорядочивающей последовательности $\left(a_{0}, \ldots, a_{n+1}\right)$. Тогда для каждого $s$ имеем:

$$
\partial_{0} W \cup B_{\delta}(\text { ind } \leqslant s ; v) \subset \phi^{-1}\left(\left[a_{0}, a_{s+1}\right]\right) \subset C_{\delta}(\text { ind } \leqslant s ; v) \cup \partial_{0} W
$$

и әти включения являются гомотопическими әквивалентностями.

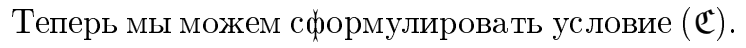

ОПРЕДЕЛЕНИЕ 4.5. Скажем, что $v$ удовлетворяет условию (ㄷ), если найдутся объекты 1)-4), перечисленные ниже, со свойствами (А), (В), указанньпии ниже.

\section{Обвекты:}

1) Упорядоченная функция Морса $\phi_{1}$ на $\partial_{1} W$ с упорядочиваюшей последовательностью $\left(\alpha_{0}, \ldots, \alpha_{n}\right)$ и $\phi_{1}$-градиентом $u_{1}$.

2) Упорядоченная функция Морса $\phi_{0}$ на $\partial_{0} W$ с упорядочивающей последовательностью $\left(\beta_{0}, \ldots, \beta_{n}\right)$ и $\phi_{0}$-градиентом $u_{0}$.

3) Упорядоченная функция Морса $\phi$ на $W$ с упорядочивающей последовательностью $\left(a_{0}, \ldots, a_{n+1}\right)$, согласованная с $(f, v)$.

4) Число $\delta>0$.

Свойства:

(А) $u_{0}$ является $\delta$-разделенным по отношению к $\phi_{0}, u_{1}$ является $\delta$-разделенным по отношению к $\phi_{1}$, и $v$ является $\delta$-разделенньм по отношению к $\phi$.

(B1) $(-v)^{\rightsquigarrow}\left(C_{\delta}\left(\right.\right.$ ind $\left.\left.\leqslant j ; u_{1}\right)\right) \cup\left(D_{\delta}(\right.$ ind $\left.\leqslant j+1 ; v) \cap \partial_{0} W\right) \subset B_{\delta}\left(\right.$ ind $\left.\leqslant j, u_{0}\right)$ для каждого $j$.

(B0) $\stackrel{\vec{v}}{ }\left(C_{\delta}\left(\right.\right.$ ind $\left.\left.\leqslant j ;-u_{0}\right)\right) \cup\left(D_{\delta}(\right.$ ind $\left.\leqslant j+1 ;-v) \cap \partial_{1} W\right) \subset B_{\delta}\left(\right.$ ind $\left.\leqslant j ;-u_{1}\right)$ для каждого $j$.

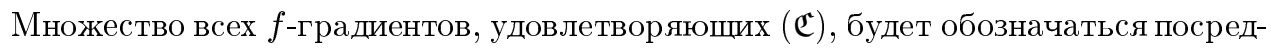
ством $\mathscr{G}(f)$. Напомним, что множество всех $f$-градиентов, удовлетворяющих условию трансверсальности, обозначается $\mathscr{G}(f)$. Пересечение $\mathscr{G} \mathfrak{C}(f) \cap \mathscr{G} \mathscr{T}(f)$ будет обозначаться $\mathscr{G} \mathfrak{C} \mathscr{T}(f)$.

\section{Комментарии:}

1. Как мы уже отметили во введении, условие (В) является аналогом условия клеточной аппроксимации. В самом деле, множества $C_{\delta}\left(\right.$ ind $\left.\leqslant j ; u_{1}\right)$ и $B_{\delta}\left(\right.$ ind $\left.\leqslant j ; u_{0}\right)$ суть “утолщения" множеств $D\left(\right.$ ind $\left.\leqslant j ; u_{1}\right)$ и, соответственно, $D\left(\right.$ ind $\left.\leqslant j ; u_{0}\right)$; причем первое из них - "толще", нежели второе. Стало быть если градиент $v$ удовлетворяет (B1), то $(-v)^{\rightsquigarrow}$ переводит некоторое утолщение $j$-остова $\partial_{1} W$ в некоторое утолщение $j$-остова $\partial_{0} W$. Предупреждение: условие (B1) требует не только этого, а несколько больше: для каждого $j$ подошвы $\delta$-ручек $W$ индекса $\leqslant j+1$ должны принадлежать множеству $B_{\delta}\left(\right.$ ind $\left.\leqslant j ; u_{0}\right)$. 
2. Условие $(\mathfrak{C})$ сильнее, нежели условие $(\mathscr{C})$ из $[6 ; \S 1.2]$. Это ясно, поскольку $(\mathfrak{C})$ формулируется точно так же, как $(\mathscr{C})$, с той лиш разницей, что в условии $(\mathscr{C})$ требуется $(-v)^{\rightsquigarrow}\left(\phi_{1}^{-1}\left(\left[a_{0}, a_{j+1}\right]\right)\right) \subset B_{\delta}\left(\mathrm{ind} \leqslant j ; u_{0}\right)$. Условие $(\mathscr{C})$ оказывается, в свою очередь, сильнее, чем условие (RP) (см. определение 3.11 ); это немного менее очевидно, и мы не приводим здесь доказательство этого факта.

3 . Если даны $\phi_{0}$-градиент $u_{0}, \phi_{1}$-градиент $u_{1}$ и $\phi$-градиент $v$, удовлетворяюший условию почти-трансверсальности, то условие (А) всегда выполнено при достаточно малом $\delta$.

Tеорема 4.6. $\mathscr{G}(f)$ открыто и плотно в $\mathscr{G}(f)$ по отношению $к C^{0}$-топологии. Более того, если $v_{0}$ - произвольный $f$-градиент, то можно выбрать $C^{0}$-малое возмущение $v$ градиента $v_{0}$ таким образом, ито $v \in \mathscr{G}(f)$ u $v=v_{0}$ в окрестности $\partial W$.

ДоКАЗАТЕЛЬСТво теоремы занимает оставшуюся часть параграфһа и содержит две части: доказательства открытости и плотности.

$C^{0}$-открытость $\mathscr{G}(f)$.

Пусть $v-f$-градиент, удовлетворяющий $(\mathfrak{C})$. Выберем соответствуюшие функции $\phi_{0}, \phi_{1}, \phi$, их градиенты $u_{0}, u_{1}$, и число $\delta>0$, удовлетворяюшие (A), (B0) и (B1). Теперь зафиксируем последовательность $\mathscr{S}=\left(\delta, u_{0}, u_{1}, \phi_{0}, \phi_{1}, \phi\right)$. Пусть $w$ есть $\phi$-градиент. Обозначим условие $(\mathrm{A})$ по отношению к $w$ и $\mathscr{S}$ посредством $(\mathrm{A})(w)$. Аналогично, обозначим условия (B1), (В0) по отношению к $w$ и $\mathscr{S}$ посредством $(\mathrm{B} 1)(w),(\mathrm{B} 0)(w)$. Мы знаем, что выполняется $(\mathrm{A})(v) \&(\mathrm{~B} 1)(v) \&(\mathrm{~B} 0)(v) ;$ теперь мы докажем, что $(\mathrm{A})(w) \&(\mathrm{~B} 1)(w) \&(\mathrm{~B} 0)(w)$ выполнено для каждого $w$, достаточно $C^{0}$-близкого к $v$.

Пусть $w$ - другой $f$-градиент. Из леммы 1.6 и следствия 1.7 работы [2] вытекает, что если $\|w-v\|$ достаточно мало, то $w$ является $\delta$-разделенным $\phi$-градиентом, так что условие $\mathrm{A}(w)$ выполнено.

Нам будет удобнее работать с другой формулировкой условия $(\mathrm{B} 1)(w) \&(\mathrm{~B} 0)(w)$. Введем еще три условия:

$\beta_{1}(w)$ Для каждого $j$ имеем: $w$ спускает $C_{\delta}\left(\right.$ ind $\left.\leqslant j ; u_{1}\right)$ до $\phi^{-1}\left(a_{j+1}\right)$ без пересечения $\mathrm{c} \bigcup_{\text {ind } p \geqslant j+1} D_{\delta}(p)$.

$\beta_{2}(w)$ Для каждого $j$ имеем: $w$ поднимает $C_{\delta}\left(\operatorname{ind} \leqslant n-j-2 ;-u_{0}\right)$ до $\phi^{-1}\left(a_{j+2}\right)$ без пересечения с $\bigcup_{\text {ind } p \leqslant j+1} D_{\delta}(p)$.

$\beta_{3}(w)$ Для каждого $j$ множества

$$
R_{j}^{+}(w)=(-v)_{\left[b, a_{j+2}\right]}^{\rightsquigarrow}\left(C_{\delta}\left(\operatorname{ind} \leqslant j ; u_{1}\right)\right)
$$

и

$$
R_{j}^{-}(w)=v_{\left[a, a_{j+2}\right]}^{\rightsquigarrow}\left(C_{\delta}\left(\text { ind } \leqslant n-j-2 ;-u_{0}\right)\right)
$$

не пересекаются.

Отметим, что $\beta_{1}(w)$ и $\beta_{2}(w)$ в некотором смысле двойственны друг к другу, т.е. $\beta_{2}(w)=\beta_{1}(-w)$.

Лемма 4.7. Предположим, что выполнено $\mathrm{A}(w)$. Тогда $(\mathrm{B} 1)(w) \&(\mathrm{~B} 0)(w) \Leftrightarrow$ $\beta_{1}(w) \& \beta_{1}(w) \& \beta_{3}(w)$. 
ДокАЗАТЕльство. $\Rightarrow$. Условие $\beta_{1}$ следует из (В0), $\beta_{2}$ следует из (В1). Чтобы доказать $\beta_{3}$, заметим, что если $R_{j}^{+}(w) \cap R_{j}^{-}(w) \neq \varnothing$, то найдется $(-v)$-траектория, соединяющая $x \in C_{\delta}\left(\right.$ ind $\left.\leqslant j ; u_{1}\right)$ с $y \in C_{\delta}$ (ind $\left.\leqslant n-j-2 ;-u_{0}\right)=\partial_{0} W \backslash B_{\delta}\left(\right.$ ind $\left.\leqslant j ; u_{0}\right)$. А это противоречит (B1).

$\Leftarrow$. Условие $\beta_{1}$ утверждает, что для каждого $j$ выполнено

$$
\left(\bigcup_{\text {ind } p \geqslant j+1} D_{\delta}(p,-v)\right) \cap \partial_{1} W \subset \partial_{1} W \backslash C_{\delta}\left(\text { ind } \leqslant j ; u_{1}\right)=B_{\delta}\left(\text { ind } \leqslant n-2-j ;-u_{1}\right),
$$

т.е. $D_{\delta}($ ind $\leqslant n-1-j ;-v) \cap \partial_{1} W \subset B_{\delta}\left(\right.$ ind $\left.\leqslant n-2-j ;-u_{1}\right)$. Далее, из $\beta_{3}$ следует, что для каждого $j$ каждая $v$-траектория, начинающаяся в $C_{\delta}\left(\right.$ ind $\left.\leqslant n-j-2 ;-u_{0}\right)$ и достигающая $\partial_{1} W$, не может пересечь $\partial_{1} W$ в точке множества $C_{\delta}\left(\right.$ ind $\left.\leqslant j ; u_{1}\right)$. Следовательно, она пересекает $\partial_{1} W$ в точке множества $B_{\delta}\left(\right.$ ind $\left.\leqslant n-2-j ;-u_{1}\right)$. Значит, вьполнено $(\mathrm{B} 0)(w)$. Доказательство $(\mathrm{B} 1)(w)$ аналогично.

Возврашаясь в доказательству $C^{0}$-открытости $(\mathfrak{C})$, заметим, что, как несложно проверить, условия $\beta_{1}(w)$ и $\beta_{2}(w)$ открыты. Поскольку выполнены $\beta_{1}(v)$ и $\beta_{2}(v)$, то для каждого $j$ множества $R_{j}^{+}(v), R_{j}^{-}(v)$ компактны. Так как они не пересекаются (что следует из $\left.\beta_{3}(v)\right)$, то можно выбрать два непересекающихся открытых подмножества $U_{j}, V_{j}$ множества $\phi^{-1}\left(a_{j+2}\right)$ таких, что $R_{j}^{+}(v) \subset U_{j}, R_{j}^{-}(v) \subset V_{j}$. Тогда для каждого $w$, достаточно $C^{0}$-близкого к $v$, и для каждого $j$ мы имеем (см. следствие 5.6 из [2]):

$$
(-w)_{\left[b, a_{j+2}\right]}^{\rightsquigarrow}\left(C_{\delta}\left(\text { ind } \leqslant j ; u_{1}\right)\right) \subset U_{j}, \quad w_{\left[a, a_{j+2}\right]}^{\rightsquigarrow}\left(C_{\delta}\left(\text { ind } \leqslant n-2-j ;-u_{0}\right)\right) \subset V_{j},
$$

и $\beta_{3}(w)$ доказано. Следовательно, $\beta_{1}(w) \& \beta_{2}(w) \& \beta_{3}(w)$ является $C^{0}$-открытым условием и, таким образом, мы доказали $C^{0}$-открытость условия $(\mathfrak{C})$.

$C^{0}$-плотность.

Доказательство очень близко к доказательству $C^{0}$-плотности условия (RP). Мы не проводим его во всех подробностях. Заметим только, что, доказывая $C^{0}$-плотность условия (RP), мы построили возмушение $v^{\prime}$ данного градиента $v$, которое на самом

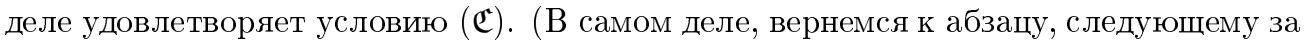
формулой $(7)$. Диффеоморфизм $\Phi_{1}^{-1}$ переводит $\partial_{1} W \backslash A_{n-2-m}^{+}(\delta)$ в множество $A_{m}^{-}(\delta)$. Поскольку $u_{0}$ и $u_{1}$ являются $\delta$-разделенными, множество $\left(\partial_{1} W\right)^{\{\leqslant m\}}$ лежит в $\partial_{1} W \backslash$ $A_{n-2-m}^{+}(\delta)$, и, таким образом, мы проверили условие (B1).)

4.3. Гомологический градиентный спуск (второй вариант). В этом пункте мы приведем еще один вариант построения оператора гомологического градиентного спуска (см. п. 3.5). Этот вариант является более подходящим для изучения морсовских фильтраций кобордизмов $(\S 5)$.

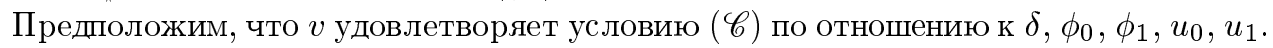
У нас имеются соответствуюшие фильтрации $\left(\partial_{1} W\right)^{\{\leqslant s\}},\left(\partial_{0} W\right)^{\{\leqslant s\}}$. Положим $V_{\alpha}=\phi^{-1}(\alpha)$.

Теорема 4.8. Для каждого s существует гомоморфизм

$$
\mathscr{H}_{s}(-v): H_{*}\left(V_{b}^{\{\leqslant s\}}, V_{b}^{\{\leqslant s-1\}}\right) \rightarrow H_{*}\left(V_{a}^{\{\leqslant s\}}, V_{a}^{\{\leqslant s-1\}}\right),
$$


обладающий следующими свойствами.

1) Пусть $N$ - ориентированное подмногообразие $V_{b}$ такое, что $N \subset V_{b}^{\{\leqslant s\}}$ u $N \backslash \operatorname{Int} V_{b}^{\{\leqslant s-1\}}$ компактно. Тогда многообразие $N^{\prime}=(-v)_{[b, a]}^{\varkappa}(N)$ лежит в $V_{a}^{\{\leqslant s\}}$ и $N^{\prime} \backslash \operatorname{Int} V_{a}^{\{\leqslant s-1\}}$ компактно, фундаментальный класс $N^{\prime}$ по модулю $V_{a}^{\{\leqslant s-1\}}$ равен $\mathscr{H}_{s}(-v)([N])$.

2) Существует $\epsilon>0$ такое, что для каждого $f$-градиента $w$, удовлетворяющего $\|w-v\| \leqslant \epsilon$, и каждого s выполнено: $\mathscr{H}_{s}(-v)=\mathscr{H}_{s}(-w)$.

ДокаЗАТЕЛЬСТво. 1) Построение гомоморфизма $\mathscr{H}_{s}(-v)$.

Гомоморфизм $\mathscr{H}_{s}(-v)$ будет определен как композиция двух гомоморфизмов: $\mathscr{H} 1_{s}(-v)$ и $\mathscr{H} 0_{s}(-v)$. (Интуитивно, $\mathscr{H} 1$ соответствует спуску с уровня $b$ на уровень $a_{s+1}$ и $\mathscr{H} 0$ соответствует спуску с $a_{s+1}$ в $a$.) Отображения $(-v)_{\left[b, a_{s+1}\right]}^{\rightsquigarrow}$ и $(-v)_{\left[a_{s+1}, a\right]}^{\rightsquigarrow ы ~ о б ~ о б о з н а ч и м ~ к а к ~}(-v 1)^{\rightsquigarrow}$, и, соответственно, $(-v 0)^{\rightsquigarrow}$. Каждая $(-v)$-траектория, начинаюшаяся в $V_{b}^{\{\leqslant s\}}$, достигает $V_{a_{s+1}}$. Поэтому $V_{b}^{\{\leqslant s\}}$ лежит в области определения $(-v 1)^{\rightsquigarrow}$, и $(-v 1)^{\rightsquigarrow}$ определяет гомеоморфизм пары $\left(V_{b}^{\{\leqslant s\}}, V_{b}^{\{\leqslant s-1\}}\right)$ на свой образ, являюшийся парой компактных подмножеств множества $V_{a_{s+1}}$.

ОПРЕДЕЛЕНИЕ 4.9. Пусть $k$ - целое, $0 \leqslant k \leqslant n$. Обозначим через $Y_{k}(v)$ множество всех $y \in \phi^{-1}\left(\left[a, a_{k+1}\right]\right)$ таких, что или

i) $\gamma(y, \cdot ;-v)$ достигает $V_{a}$ и пересекает его в точке $z \in V_{a}^{\{\leqslant k-1\}}$, или

ii) $\lim _{t \rightarrow \infty} \gamma(y, t ;-v)=p$, где $p \in S(f)$.

Другими словами,

$$
Y_{k}(v)=T\left(V_{a}^{\{\leqslant k-1\}},-v \mid W^{\prime}\right) \cup D\left(-v \mid W^{\prime}\right),
$$

где $W^{\prime}=\phi^{-1}\left(\left[a, a_{k+1}\right]\right)$. Множество $Y_{k}(v)$ компактно (см. [2; лемма 2.7]). Положим

(8) $\quad A=Y_{s+1}(v) \cap V_{a_{s+1}}, \quad B=Y_{s}(v) \cap V_{a_{s+1}}, \quad C=\left(\bigcup_{\text {ind } p \leqslant s} D(p,-v)\right) \cap V_{a_{s+1}}$.

Тогда $C$ замкнуто, $\bar{C} \subset \operatorname{Int} B$ и $A \supset B \supset \operatorname{Int} B \supset C$. Далее, отображение $(-v 1)^{\rightsquigarrow}$ определяет непрерьвное отображение пар

$$
\left(V_{b}^{\{\leqslant s\}}, V_{b}^{\{\leqslant s-1\}}\right) \rightarrow(A, B),
$$

а отображение $(-v 0)^{\rightsquigarrow}$ определяет непрерьвное отображение пар

$$
(A \backslash C, B \backslash C) \rightarrow\left(V_{a}^{\{\leqslant s\}}, V_{a}^{\{\leqslant s-1\}}\right) .
$$

Обозначим символом Ехс изоморфизм вырезания $H_{*}(A \backslash C, B \backslash C) \rightarrow H_{*}(A, B)$ и положим

$$
\mathscr{H}_{s}(-v)=\left((-v 0)^{\rightsquigarrow} \circ \operatorname{Exc}^{-1} \circ(-v 1)^{\rightsquigarrow}\right)_{*} .
$$


2) Доказательство свойств $\mathscr{H}_{s}(-v)$.

Доказательство сводится к свойствам оператора $H(v)$, построенного по некоторой $R$-системе (см. п. 3.5).

Мы предъявим $R$-систему $\mathscr{R}=\left\{\left(A_{\lambda}, B_{\lambda}\right)\right\}_{\lambda \in \Lambda}$ для $(\phi, v)$ такую, что гомоморфизм $H(v)$, соответствуюший $\mathscr{R}$, является изоморфным $\mathscr{H}(-v)$. Чтобы объяснить конструкцию $\mathscr{R}$, сделаем упрощающее предположение: $\phi$ имеет только два критических значения: первое - в $\left[a, a_{s+1}\right]$ и второе - в $\left[a_{s+1}, b\right]$. (В конце доказательства мы укажем, как перенести его на общий случай.) Положим

$$
\begin{array}{ll}
A_{b}=V_{b}^{\{\leqslant s-1\}}, & A_{a}=V_{a}^{\{\leqslant s-1\}}, \\
B_{a}=V_{a}^{\{\geqslant s+1\}}, & B_{b}=V_{b}^{\{\geqslant s+1\}} .
\end{array}
$$

Для краткости обозначим $a_{s+1}$ символом $\tau$. Для построения $R$-системы нужно предъявить множества $A_{\tau}, B_{\tau}$. Пусть $\left(\alpha_{i}\right)$ - упорядочивающая последовательность для $\phi_{1}$, $\left(\beta_{i}\right)$ - упорядочивающая последовательность для $\phi_{0}$. Выберем число $\epsilon>0$, достаточно малое, чтобы было выполнено следующее:

$$
\begin{aligned}
& \left.\left.D_{\delta}\left(\mathrm{ind} \leqslant n-s-2,-u_{1}\right) \subset \phi_{1}^{-1}(] \alpha_{s+1}+\epsilon, \alpha_{n}\right]\right) \text {, } \\
& \alpha_{s}+\epsilon<\alpha_{s+1}, \\
& (-v)^{\rightsquigarrow}\left(\phi_{1}^{-1}\left(\left[\alpha_{0}, \alpha_{s}+\epsilon\right]\right)\right) \subset \phi_{0}^{-1}\left(\left[\beta_{0}, \beta_{s}[),\right.\right.
\end{aligned}
$$

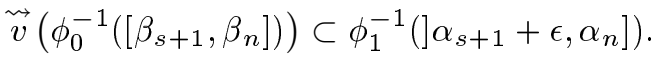

Символом $L_{s}(v)$ обозначим множество всех точек $y \in \phi^{-1}\left(\left[a_{s+1}, b\right]\right)$ таких, что или

i) $\gamma(y, \cdot ; v)$ достигает $V_{b}$ и пересекает его в точке $z \in \phi^{-1}\left(\left[\alpha_{s+1}+\epsilon, \alpha_{n}\right]\right)$, или

ii) $y \in D(p, v)$, где $p \in S(f)$.

Теперь положим

$$
A_{\tau}=(-v 1)^{\rightsquigarrow}\left(\phi_{1}^{-1}\left(\left[\alpha_{0}, \alpha_{s}+\epsilon\right]\right)\right), \quad B_{\tau}=L_{s}(v) \cap V_{\tau} .
$$

Из условия (ㄷ) следует, что $\left\{\left(A_{\lambda}, B_{\lambda}\right)\right\}_{\lambda \in\{a, \tau, b\}}-R$-система для $(\phi, v)$.

Вложения пар

$$
\begin{aligned}
& j_{b}:\left(V_{b}^{\{\leqslant s\}}, V_{b}^{\{\leqslant s-1\}}\right) \subset\left(V_{b} \backslash B_{b}, A_{b}\right), \\
& j_{a}:\left(V_{a}^{\{\leqslant s\}}, V_{a}^{\{\leqslant s-1\}}\right) \subset\left(V_{a} \backslash B_{a}, A_{a}\right)
\end{aligned}
$$

индуцируют изоморфизмы в гомологиях.

Проследив еше раз построение гомоморфизма $H(v)$, соответствующего $R$-системе $\left\{\left(A_{\lambda}, B_{\lambda}\right)\right\}_{\lambda \in \Lambda}$, нетрудно убедиться, что $H(v) \circ\left(j_{b}\right)_{*}=\left(j_{a}\right)_{*} \circ \mathscr{H}(-v)$. Теперь уже легко вывести свойства гомоморфизма $\mathscr{H}(-v)$ из свойств $H(v)$.

В случае, когда найдется более чем одно критическое значение в отрезках $\left[a_{s+1}, b\right]$ и $\left[a, a_{s+1}\right]$, действуем аналогично: строим $R$-систему $\mathscr{R}=\left\{\left(A_{\lambda}, B_{\lambda}\right)\right\}_{\lambda \in \Lambda}$ такую, что оператор $H(-v)$, соответствующий $\mathscr{R}$, изоморфен $\mathscr{H}(-v)$. В этой системе мы можем определить $A_{\lambda}, B_{\lambda}$ по тем же формулам, что и вьше для $\lambda=a, b, \tau$. Чтобы определить 
$A_{\lambda}, B_{\lambda}$ для остальных значений $\lambda$, сперва выберем множество $\Lambda=\left\{\lambda_{i}\right\}$ регулярных значений такое, что $\lambda_{0}=a, \lambda_{N}=b, \lambda_{i}=\tau$ и для каждого $i$ найдется лишш одно критическое значение $\phi$ в $\left[\lambda_{i}, \lambda_{i+1}\right]$.

Множество $A_{\lambda_{j}}$ при $i \leqslant j \leqslant N$ определяется как некоторое утолщение множества $(-v)_{\left[b, \lambda_{j}\right]}^{\rightsquigarrow}\left(V_{b}^{\{\leqslant s-1\}}\right)$.

Множество $B_{\lambda_{j}}$ при $i \leqslant j \leqslant N$ определяется аналогично множеству $L_{s}(v)$ (см. вьшше). Определение множеств $A_{\lambda}, B_{\lambda}$ для оставшихся значений $\lambda$, а также проверку изоморфности получаюшегося гомоморфизма $H(v)$ и гомоморфизма $\mathscr{H}(-v)$ мы оставляем читателю.

4.4. Эквивариантный гомологический градиентный спуск. Пусть $p: \widehat{W} \stackrel{H}{\longrightarrow} W$ - регулярное накрытие $W$ со структурной группой $H$. Для $A \subset W$ обозначим $p^{-1}(A)$ также символом $\widehat{A}$. Перенос вдоль $(-v)$-траекторий определяет диффеоморфизм $(-\widehat{v})^{\rightsquigarrow}:\left(\partial_{0} \widehat{W \backslash K_{1}}\right) \rightarrow\left(\partial_{0} \widehat{W \backslash K} K_{0}\right)$ (определение $K_{1}, K_{0}$ см. в п. 2.1), который коммутирует с правым действием $G$. Как и раньше, будем писать $(-\widehat{v})^{\rightsquigarrow}(X)$ вместо $(-\widehat{v})^{\rightsquigarrow}\left(X \backslash \widehat{K}_{1}\right)$. Предположим, что $v$ удовлетворяет условию $(\mathfrak{C})$ по отношению к $\delta, \phi_{0}, \phi_{1}, u_{1}, u_{0}$. Обозначим $\phi^{-1}(\alpha)$ символом $V_{\alpha}$. Оказьвается, что можно определить "эквивариантный аналог" оператора гомологического градиентного спуска. Свойства этого оператора составляют содержание следуюшей теоремы. Доказательство полностью аналогично доказательству теоремы 4.8 и не приводится.

Теорема 4.10. Для кажддого s существует гомоморфизм правых $\mathbb{Z} H$-модулей

$$
\widehat{\mathscr{H}}_{s}(-v): H_{*}\left(\widehat{V}_{b}^{\{\leqslant s\}}, \widehat{V}_{b}^{\{\leqslant s-1\}}\right) \rightarrow H_{*}\left(\widehat{V}_{a}^{\{\leqslant s\}}, \widehat{V}_{a}^{\{\leqslant s-1\}}\right),
$$

обладающий следующими свойствами.

Пусть $N$ - ориентированное подмногообразие $\widehat{V}_{b}$ такое, что $N \subset \widehat{V}_{b}^{\{\leqslant s\}} u$ $N \backslash \operatorname{Int} \widehat{V}_{b}^{\{\leqslant s-1\}}$ компактно.

Тогда $N^{\prime}=(-\widehat{v})_{[b, a]}^{\rightsquigarrow}(N)-$ подмногообразие $\left(\widehat{V_{a} \backslash B_{a}}\right)$ такое, ито $N^{\prime} \subset \widehat{V}_{a}^{\{\leqslant s\}}$ и $N^{\prime} \backslash \widehat{V}_{a}^{\{\leqslant s-1\}}$ компактно, и фундаментальный класс $N^{\prime}$ по модулю $\widehat{V}_{a}^{\{\leqslant s-1\}}$ coвnадает с $\widehat{\mathscr{H}}_{s}(-v)([N])$.

4.5. Циклические кобордизмы и условия (다). Результаты этого пункта будут использованы в $\S \S 5-7$.

ОПРЕДЕЛЕНИЕ 4.11. Циклический кобордизм - это риманов кобордизм $W$ вместе с изометрией $\Phi: \partial_{0} W \rightarrow \partial_{1} W$.

Пусть $f: W \rightarrow[a, b]$ - функция Морса на циклическом кобордизме $W$ размерности $n, v-f$-градиент. Скажем, что $v$ удовлетворяет $(\mathfrak{C} \mathscr{Y})$, если $v$ удовлетворяет условию (ㄷ) (см. п. 4.2) и, вдобавок, функции Морса $\phi_{1}, \phi_{0}$ и их градиенты $u_{1}, u_{0}$ из определения 4.5 могут быть выбраны таким образом, что $\phi_{1} \circ \Phi=\phi_{0}, u_{1}=(\Phi)_{*}\left(u_{0}\right)$. Множество всех $f$-градиентов, удовлетворяюших $(\mathfrak{C} \mathscr{Y})$, будет обозначаться $\mathscr{G} \mathfrak{C} \mathscr{Y}(f)$. Пересечение $\mathscr{G} \mathfrak{C} \mathscr{Y}(f) \cap \mathscr{G} \mathscr{T}(f)$ будет обозначаться $\mathscr{G} \mathfrak{C} \mathscr{T}(f)$. 
TЕОрема 4.12. $\mathscr{G} \mathscr{C}(f)$ открыто и плотно в $\Gamma(f)$ по отношению $\kappa C^{0}$-топологии. Более того, если $v_{0}-$ произвольный $f$-градиент, тогда можсно выбрать такое $C^{0}$-малое возмущение $v$ градиента $v_{0}$, что $v \in \mathscr{G} \mathfrak{C}(f) u v=v_{0}$ в окрестности $\partial W$.

ДокАЗАТЕЛЬСТВО в точности повторяет доказательство теоремы 4.6. Чтобы доказать $C^{0}$-открытость множества $\mathscr{G} \mathfrak{C}(f)$, можно в точности следовать рассуждениям в первой части доказательства теоремы 4.6. Для доказательства $C^{0}$-плотности, снова вернемся к доказательству $C^{0}$-плотности (RP) (теорема 3.12 ). Здесь достаточно заметить, что мы можем выбрать $u_{1}, u_{0}, \xi_{1}, \xi_{0}, \phi_{1}, \phi_{0}$ таким образом, чтобы $u_{1}=\Phi_{*}\left(u_{0}\right), \xi_{1}=\Phi_{*}\left(\xi_{0}\right), \phi_{0}=\phi_{1} \circ \Phi$.

Для $f$-градиента $v$, удовлетворяющего ( $\mathscr{Y})$, мы можем превратить оператор гомологического градиентного спуска в гомоморфизм некоторой абелевой групшы в себя. А именно, положим $h_{s}(-v)=\mathscr{H}_{s}(-v) \circ \Phi_{*}$; тогда $h_{s}(-v)-$ эндоморфизм групшы $H_{*}\left(\left(\partial_{0} W\right)^{\{\leqslant s\}},\left(\partial_{0} W\right)^{\{\leqslant s-1\}}\right)$.

\section{$\S 5$. Морсовские фильтрации кобордизмов}

Пусть $W$ - кобордизм, $\phi: W \rightarrow[a, b]$ - упорядоченная функция Морса на $W, v-$ $\phi$-градиент. Предположим, что $W$ риманов и что $v-\delta$-разделен. В п. 4.1 мы рассмотрели $H$-фильтрацию $W$, ассоциированную с $v$. Зная гомологии последовательных факторов каждой из этих фильтраций, можно в принципе восстановить гомологии пары $\left(W, \partial_{0} W\right)$. Но невозможно восстановить гомологии самого кобордизма $W$. (В самом деле, рассмотрим следующий пример: $W=V \times[0,1]$. Здесь все члены фильтрации гомотопически эквивалентны $V$, и факторы стягиваемы.) В настоящем параграфе мы определяем другую фильтрацию $W$, которая в некотором смысле согласована с данными фильтрациями многообразий $\partial_{0} W$ и $\partial_{1} W$ и исходя из которой можно восстановить гомологии кобордизма $W$. Чтобы построить такую фильтрацию, нужно наложить условие $(\mathfrak{C})$ на поле $v$.

5.1. Определение фильтрации морсовского типа. Пусть $W$ - риманов кобордизм размерности $n, f: W \rightarrow[a, b]$ - функция Морса на $W$ и $v-f$-градиент. Пред-

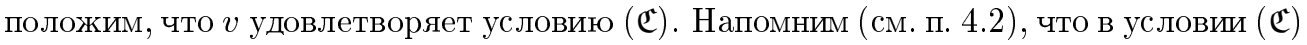
требуется сушествование упорядоченных функций Морса $\phi: W \rightarrow[a, b], \phi_{1}: \partial_{1} W \rightarrow \mathbb{R}$, $\phi_{0}: \partial_{0} W \rightarrow \mathbb{R}, \delta$-разделенного $\phi_{0}$-градиента $u_{0}$ и $\delta$-разделенного $\phi_{1}$-градиента $u_{1}$, причем эти объекты должны удовлетворять условиям (A), (B1), (B0) из п. 4.2. Мы получаем три $H$-фильтрации, построенные по упорядоченным функциям Морса $\phi_{0}, \phi_{1}$ и $\phi$ : фильтрация $\left(\partial_{1} W\right)^{\{\leqslant s\}}$ многообразия $\partial_{1} W$, фильтрация $\left(\partial_{0} W\right)^{\{\leqslant s\}}$ многообразия $\partial_{0} W$ и фильтрация $W^{\{\leqslant s\}}$ многообразия $W$.

Напомним определение множества

$$
Y_{k}(v)=\left(T\left(\left(\partial_{0} W\right)^{\{\leqslant k-1\}},-v\right) \cup D(-v)\right) \cap \phi^{-1}\left(\left[a, a_{k+1}\right]\right)
$$

из предыдущего параграфа (определение 4.9). В этом параграфе мы будем иметь дело только с одним $f$-градиентом, так что мы будем писать $Y_{k}$ вместо $Y_{k}(v)$.

ОПРЕДЕЛЕНИЕ 5.1. Пусть $A \subset W$ и $c, d \in[a, b]$. Обозначим $A \cap \phi^{-1}([c, d])$ символом $\left.A\right|_{[c, d]}$. Пусть $\mu \in[a, b]$. Обозначим $A \cap \phi^{-1}(\mu)$ символом $\left.A\right|_{\mu}$. 

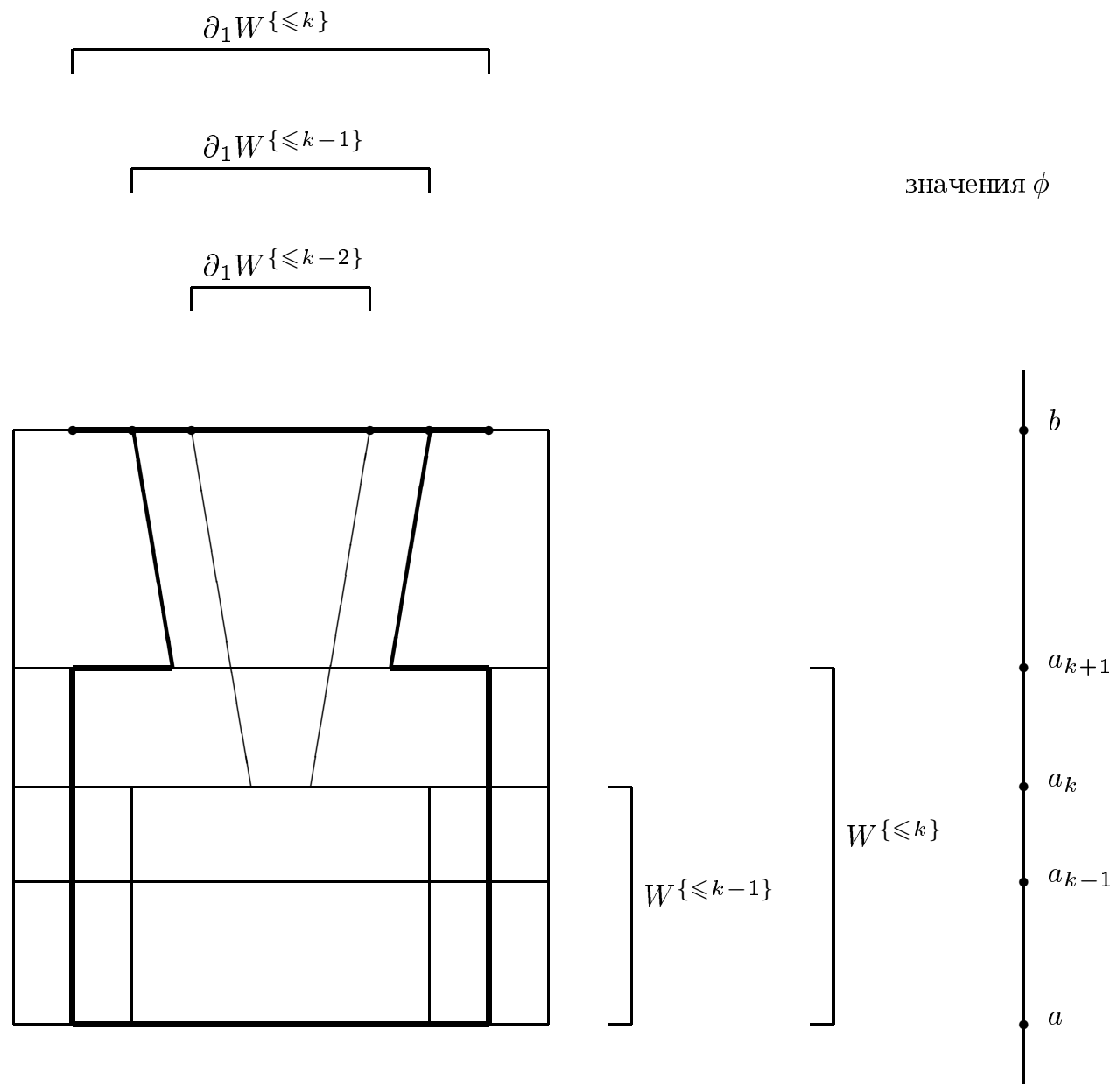

Рис. 1. $W^{\langle k\rangle}$

\section{Положим}

(9)

$$
Z_{k}=\left.\left(T\left(\left(\partial_{1} W\right)^{\{\leqslant k-1\}}, v\right)\right)\right|_{\left[a_{k+1}, b\right]} .
$$

Так как $(-v)$ спускает $\left(\partial_{1} W\right)^{\{\leqslant k-1\}}$ до $\phi^{-1}\left(a_{k}\right)$ (что следует из $\left.(\mathfrak{C})\right)$, то множество $Z_{k}$ гомеоморфно произведению $\left(\partial_{1} W\right)^{\{\leqslant k-1\}} \times[0,1]$.

Для $k \geqslant 0$ положим

$$
\begin{aligned}
W^{\prec k \succ} & =\left(\partial_{0} W\right)^{\{\leqslant k\}} \cup\left(\partial_{1} W\right)^{\{\leqslant k\}} \cup Y_{k} \cup Z_{k}, \\
W^{\langle k\rangle} & =\left(\partial_{1} W\right)^{\{\leqslant k\}} \cup\left(Y_{k+1} \cap \phi^{-1}\left(\left[a, a_{k+1}\right]\right)\right) \cup Z_{k} .
\end{aligned}
$$

Как $\left\{W^{\langle k\rangle}\right\}_{k \in \mathbb{N}}$, так и $\left\{W^{\prec k \succ}\right\}_{k \in \mathbb{N}}-$ фильтрации $W$, и $W^{\prec k \succ} \subset W^{\langle k\rangle}$. Множество $W^{\langle k\rangle}$ получено из $W^{\prec k \succ}$ добавлением всех точек из $W_{\left[a, a_{k+1}\right]}$, лежащих на $v$-траекториях, начинаюшихся в $\left(\partial_{0} W\right)^{\{\leqslant k\}} \backslash\left(\partial_{0} W\right)^{\{\leqslant k-1\}}$.

На рис. 1 и 2 изображены (весьма схематично) множества $W^{\langle k\rangle}$ и $W^{\langle k-1\rangle}$. 


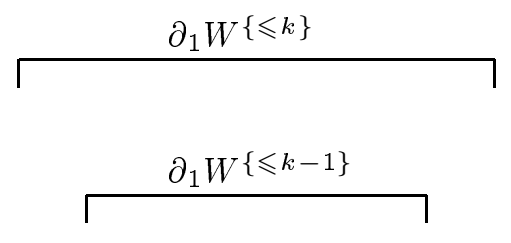

значения $\phi$

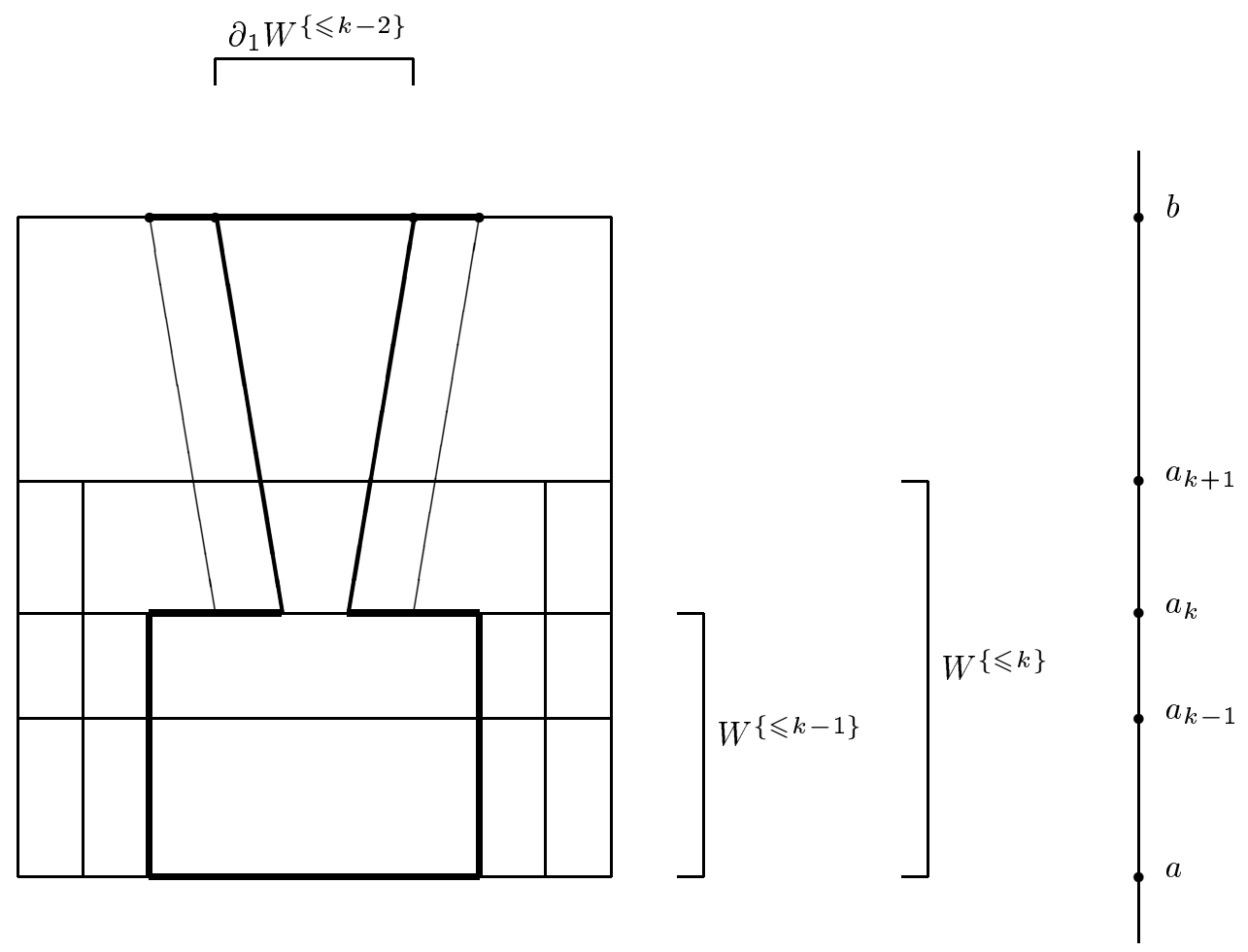

Рис. 2. $W^{\langle k-1\rangle}$

\section{ЗАМЕЧАнИЕ 5.2.}

1. Введенные выше фильтрации можно описать следуюшим образом. Будем считать, что нисходящие диски $D(p, v)$ критических точек $p$ индекса $k$ функции $f$ образуют " $k$-клетки" кобордизма $W$. Тогда множество $W \prec k \succ$ содержит все "клетки" многообразий $\partial_{0} W, \partial_{1} W, W$, имеюшие размерность $\leqslant k$, а также $v$-следы "клеток" многообразия $\partial_{1} W$, имеющих размерность $\leqslant k-1$. Множества $W^{\prec k \succ}, W^{\langle k\rangle}$ являются утолшениями этих "остовов". Условие $(\mathfrak{C})$ гарантирует, что граница клеток, соответствующих $D(p, v), p \in S(\phi)$, принадлежит объединению утолшенных клеток размерности $<$ ind $p$.

2. Две введенные выше фильтрации гомотопически эквивалентны друг другу (см. лемму 5.3). Множество $W^{\prec k \succ}$ было введено в [6] и обозначалось там символом $W^{\langle k\rangle}$. Фильтрация $\left\{W^{\langle k\rangle}\right\}_{k \in \mathbb{N}}$ имеет некоторые технические преимущества перед $\left\{W^{\prec k \succ}\right\}_{k \in \mathbb{N}}$ - вот почему мы изменили наши обозначения и почему в настоящей работе мы в основном будем рассматривать фильтрацию $W^{\langle k\rangle}$.

Положим $W^{\prec k \succ}=W^{\langle k\rangle}=\varnothing$ для $k<0$. 
Лемма 5.3. Включение $\left(W^{\prec k \succ}, W^{\prec k-1 \succ}\right) \hookrightarrow\left(W^{\langle k\rangle}, W^{\langle k-1\rangle}\right)$ является гомотопической эквивалентностью.

\section{ДокАЗАТЕЛЬСТво. Имеем}

$$
W^{\langle k\rangle} \backslash W^{\prec k \succ}=\left(Y_{k+1} \cap W^{\{\leqslant k\}}\right) \backslash\left(Y_{k} \cup\left(\partial_{0} W\right)^{\{\leqslant k\}}\right) .
$$

В области $A=\phi^{-1}\left(\left[a, a_{k+1}\right]\right) \backslash Y_{k}$ функция $\phi$ не имеет критических точек. Следовательно, найдется такое $T>0$, что для каждого $x \in A$ мы имеем: $\gamma(x, T ;-v) \in \partial_{0} W \cup$ $Y_{k}$. Для $x \in W$ символом $\tau(x)$ обозначим тот момент, когда $\gamma(x, \cdot ;-v)$ достигает $\partial_{0} W$ (если траектория не достигает $\partial_{0} W$, то положим $\tau(x)=\infty$ ). Определим

$$
\begin{aligned}
W_{0}^{\langle k\rangle} & =W^{\langle k\rangle} \cap \phi^{-1}\left(\left[a, a_{k+1}\right]\right), \\
W_{0}^{\prec k \succ} & =W^{\prec k \succ} \cap \phi^{-1}\left(\left[a, a_{k+1}\right]\right) .
\end{aligned}
$$

Определим деформацию $H_{t}$ пространства $W_{0}^{\langle k\rangle}$ в себя (где $\left.t \in[0, T]\right)$ следуюшим образом:

$$
H_{t}(x)= \begin{cases}\gamma(x, t ;-v), & \text { если } t \leqslant \min (T, \tau(x)), \\ \gamma(x, \min (T, \tau(x)) ;-v), & \text { если } t \geqslant \min (T, \tau(x)) .\end{cases}
$$

Легко убедиться в том, что $H_{0}=\mathrm{id}, H_{T}\left(W_{0}^{\langle k\rangle}\right) \subset W_{0}^{\prec k \succ}$ и что $W_{0}^{\prec k \succ}$ инвариантно относительно $H_{t}$ при всех $t \geqslant 0$. Поэтому включение $W_{0}^{\prec k \succ} \hookrightarrow W_{0}^{\langle k\rangle}$ является гомотопической эквивалентностью.

Теперь несложно продолжить деформацию $H_{t}$ на все $W^{\langle k\rangle}$, положив $H_{t}(x)=x$ для $x \in \phi^{-1}\left(\left[a_{k+1}+\epsilon, b\right]\right)$ и соответствуюшим образом склеив два отображения в области $\phi^{-1}\left(\left[a_{k+1}, a_{k+1}+\epsilon\right]\right)$. Таким образом, мы получили отображение, гомотопически обратное включению $W^{\prec k \succ} \hookrightarrow W^{\langle k\rangle}$.

Гомотопически обратное отображение для включения пар, фигурирующего в формулировке леммы, строится подобньм образом (значение $T$, возможно, придется увеличить). Мы оставляем детали читателю.

ЗАМЕЧАнИЕ 5.4. В настоящем параграфе мы будем неоднократно использовать метод, примененный при доказательстве леммы 5.3. Мы будем говорить, что мы применяем $(-v)$-сдвиг. В частности, при доказательстве леммы 5.3 мы применили $(-v)$-сдвиг в области $\phi^{-1}\left(\left[a, a_{k+1}\right]\right) \backslash Y_{k}$.

5.2. Гомологии пары $\left(W^{\langle k\rangle}, W^{\langle k-1\rangle}\right)$. Здесь мы покажем, что $W^{\langle k\rangle}$ образует клеточную фильтрацию $W$, и вычислим гомологии $\left(W^{\langle k\rangle}, W^{\langle k-1\rangle}\right)$. Замечание 5.2 подсказывает, что для $* \neq k$ группа $H_{*}\left(W^{\langle k\rangle}, W^{\langle k-1\rangle}\right)$ должна обрашаться в нуль и что при $*=k$ эта группа должна быть свободной абелевой группй, порожденной гомологическими классами соответствующих клеток. В этом параграфе мы убедимся, что это действительно выполняется.

Чтобы вычислить $H_{*}\left(W^{\langle k\rangle}, W^{\langle k-1\rangle}\right)$, мы сначала определим четыре гомоморфизма со значениями в $H_{k}\left(W^{\langle k\rangle}, W^{\langle k-1\rangle}\right)$. В теореме 5.5 мы установим, что группа $H_{k}\left(W^{\langle k\rangle}, W^{\langle k-1\rangle}\right)$ является прямой суммой образов этих гомоморфизмов. 
1. Для каждого $s$ множества $\left(\partial_{0} W\right)^{\{\leqslant s\}},\left(\partial_{1} W\right)^{\{\leqslant s\}}$ являются подмножествами $W^{\langle s\rangle}$. Следовательно, имеются гомоморфизмы, индуцированные соответствующими включениями:

$$
\begin{aligned}
& C_{k}\left(u_{0}\right)=H_{k}\left(\left(\partial_{0} W\right)^{\{\leqslant k\}},\left(\partial_{0} W\right)^{\{\leqslant k-1\}}\right) \stackrel{\mathscr{J}_{0}}{\longrightarrow} H_{k}\left(W^{\langle k\rangle}, W^{\langle k-1\rangle}\right), \\
& C_{k}\left(u_{1}\right)=H_{k}\left(\left(\partial_{1} W\right)^{\{\leqslant k\}},\left(\partial_{1} W\right)^{\{\leqslant k-1\}}\right) \stackrel{\mathscr{J}_{1}}{\longrightarrow} H_{k}\left(W^{\langle k\rangle}, W^{\langle k-1\rangle}\right) .
\end{aligned}
$$

2. Каждая $(-v)$-траектория, начинающаяся в $\left(\partial_{1} W\right)^{\{\leqslant k-1\}}$, достигает $\phi^{-1}\left(a_{k}\right)$. Поэтому сушествует непрерывное отображение

$$
G_{k}:\left(\partial_{1} W\right)^{\{\leqslant k-1\}} \times\left[a_{k}, b\right] \rightarrow \phi^{-1}\left(\left[a_{k}, b\right]\right),
$$

являюшееся гомеоморфизмом на свой образ, причем для каждого $x$ кривая $t \mapsto G_{k}(x, t)$ является репараметризованной $v$-траекторией, и $\phi\left(G_{k}(x, t)\right)=t$. Положим $I_{k}=\left[a_{k}, b\right]$. Мы получаем отображение пар

$$
G_{k}:\left(\left(\partial_{1} W\right)^{\{\leqslant k-1\}},\left(\partial_{1} W\right)^{\{\leqslant k-2\}}\right) \times\left(I_{k}, \partial I_{k}\right) \rightarrow\left(W^{\langle k\rangle}, W^{\langle k-1\rangle}\right) .
$$

Зададим гомоморфизм

$$
\mathscr{S}: C_{k-1}\left(u_{1}\right) \rightarrow H_{*}\left(W^{\langle k\rangle}, W^{\langle k-1\rangle}\right)
$$

формулой $\mathscr{S}(x)=\left(G_{k}\right)_{*}(x \otimes \iota)$ (где $\otimes-$ произведение Кюннета и $\iota$ - фундаментальный класс пары $\left.\left(I_{k}, \partial I_{k}\right)\right)$.

3. Пусть $p \in S_{k}(\phi)$. Напомним, что $d(p)$ обозначает вложенный диск $D(p, v) \cap$ $\phi^{-1}\left(\left[a_{k}, b\right]\right)$. Из условия $(\mathfrak{C})$ следует, что $\partial d(p) \subset W^{\langle k-1\rangle}$. Мыполучаем гомоморфизм

$$
C_{k}(v)=H_{k}\left(W^{\{\leqslant k\}}, W^{\{\leqslant k-1\}}\right) \stackrel{\mathscr{I}}{\longrightarrow} H_{k}\left(W^{\langle k\rangle}, W^{\langle k-1\rangle}\right) .
$$

\section{TEOPEMA 5.5 .}

1. $H_{*}\left(W^{\langle k\rangle}, W^{\langle k-1\rangle}\right)=0$, ecлu $* \neq k$.

2. Гомоморфизм

$$
\begin{aligned}
L_{k}=\left(\mathscr{J}_{1}, \mathscr{J}_{0}, \mathscr{I}, \mathscr{S}\right): C_{k}\left(u_{1}\right) \oplus C_{k}\left(u_{0}\right) \oplus C_{k}(v) \oplus C_{k-1}\left(u_{1}\right) \\
\\
\rightarrow H_{k}\left(W^{\langle k\rangle}, W^{\langle k-1\rangle}\right)
\end{aligned}
$$

является изоморфизмом.

ЗАмЕчАниЕ 5.6. Из теоремы 5.5 следует, в частности, что $H_{k}\left(W^{\langle k\rangle}, W^{\langle k-1\rangle}\right)$ является свободной абелевой группой ранга $\sharp S_{k}\left(\phi_{1}\right)+\sharp S_{k}\left(\phi_{0}\right)+\sharp S_{k}(\phi)+\sharp S_{k-1}\left(\phi_{1}\right)$. 
ДокАЗАТЕЛЬСТво. Зафиксируем значение $k$ и положим

$$
a_{k}=\lambda, \quad a_{k+1}=\mu, \quad Z=\left.T\left(\left(\partial_{1} W\right)^{\{\leqslant k-1\}}, v\right)\right|_{[\lambda, b]}
$$

и, далее,

$$
\begin{gathered}
\Delta=\bigcup_{p \in S_{k}(\phi)} d(p), \\
R=\left.Z \cup \Delta \cup\left(\partial_{1} W\right)^{\{\leqslant k\}} \cup Y_{k+1}\right|_{\lambda}, \\
S=\left.W^{\langle k-1\rangle}\right|_{[\lambda, b]},
\end{gathered}
$$

так что $S \subset R \subset \phi^{-1}([\lambda, b])$. В следующей лемме изучение гомологий $\left(W^{\langle k\rangle}, W^{\langle k-1\rangle}\right)$ сводится к изучению пересечения этой пары с $\phi^{-1}([\lambda, b])$.

\section{Лемма 5.7. Включение пар}

$$
\begin{aligned}
& (R, S) \hookrightarrow\left(\left.W^{\langle k\rangle}\right|_{[\lambda, b]},\left.W^{\langle k-1\rangle}\right|_{[\lambda, b]}\right) \hookrightarrow\left(W^{\langle k\rangle}, W^{\langle k-1\rangle}\right), \\
& \left(\Delta \cup\left(\left.Y_{k+1}\right|_{\left[a_{k}\right]}\right),\left.Y_{k}\right|_{\left[a_{k}\right]}\right) \hookrightarrow\left(\left.W^{\langle k\rangle}\right|_{[\lambda, \mu]},\left.W^{\langle k-1\rangle}\right|_{[\lambda, \mu]}\right)
\end{aligned}
$$

индуиирует изоморфизм в гомологиях.

ДокАЗАТЕЛЬСТво. Мы покажем, что (24) индуцирует изоморфизм в гомологиях. (Включения из (23) рассматриваются аналогично.) Используя $(-v)$-сдвиг в области $\left.\left.W^{\langle k\rangle}\right|_{[\lambda, \mu]} \backslash W^{\langle k-1\rangle}\right|_{[\lambda, \mu]}$, легко доказать, что включение

$$
\left(\left.\left.Y_{k}\right|_{[\lambda, \mu]} \cup Y_{k+1}\right|_{\lambda},\left.Y_{k}\right|_{\lambda}\right) \hookrightarrow\left(\left.W^{\langle k\rangle}\right|_{[\lambda, \mu]},\left.W^{\langle k-1\rangle}\right|_{[\lambda, \mu]}\right)
$$

является гомологической эквивалентностью. Пересечение множеств $\left.Y_{k}\right|_{[\lambda, \mu]}$ и $\left.Y_{k+1}\right|_{\lambda}$ лежит в $\left.Y_{k}\right|_{\lambda}$, так что пространство $\left(\left.\left.Y_{k}\right|_{[\lambda, \mu]} \cup Y_{k+1}\right|_{\lambda}\right) /\left.Y_{k}\right|_{\lambda}$ гомеоморфно букету

$$
\left.Y_{k}\right|_{[\lambda, \mu]} /\left.\left.Y_{k}\right|_{\lambda} \vee Y_{k+1}\right|_{\lambda} /\left.Y_{k}\right|_{\lambda}
$$

Включение $\left.Y_{k}\right|_{[\lambda, \mu]} /\left.Y_{k}\right|_{\lambda} \hookrightarrow \phi^{-1}([\lambda, \mu]) / \phi^{-1}(\lambda)$ также является гомологической эквивалентностью (чтобы убедиться в этом, надо применить $(-v)$-сдвиг в области $\left.\phi^{-1}([\lambda, \mu]) \backslash Y_{k}\right|_{[\lambda, \mu]}$, а затем - вырезание). Пространство $\left(\left.\Delta \cup Y_{k+1}\right|_{\lambda}\right) /\left.Y_{k}\right|_{\lambda}$ гомеоморфно букету

$$
(\Delta / \partial \Delta) \vee\left(\left.Y_{k+1}\right|_{\lambda} /\left.Y_{k}\right|_{\lambda}\right) .
$$

Стандартные рассуждения из теории Морса позволяют отождествить гомотопический тип пары $\left(\phi^{-1}([\lambda, \mu]), \phi^{-1}(\lambda)\right)$ с гомотопическим типом пары $(\Delta, \partial \Delta)$, что и завершает доказательство.

Итак, остается вычислить гомотопический тип $R / S$. Положим

$$
\rho=\left.R\right|_{\lambda}, \quad \sigma=\left.S\right|_{\lambda} .
$$


Заметим, что $R$ получено из $S$ приклеиванием четырех компактных подмножеств: $\phi_{1}^{-1}\left(\left[\alpha_{k}, \alpha_{k+1}\right]\right), G_{k}\left(\phi_{1}^{-1}\left(\left[\alpha_{k-1}, \alpha_{k}\right]\right) \times[\lambda, b]\right), \Delta, \rho$. Каждые два из них пересекаются по некоторому подмножеству множества $S$. Следовательно,

$R / S=\left(\left(\partial_{1} W\right)^{\{\leqslant k\}} /\left(\partial_{1} W\right)^{\{\leqslant k-1\}}\right) \vee \Sigma\left(\left(\partial_{1} W\right)^{\{\leqslant k-1\}} /\left(\partial_{1} W\right)^{\{\leqslant k-2\}}\right) \vee((\Delta \cup \rho) / \sigma)$, где $\Sigma$ - надстройка. Теперь мы займемся описанием пространства $(\Delta \cup \rho) / \sigma$. Так как $\Delta \cap \rho \subset \sigma$, то имеется гомеоморфизм

$$
(\Delta / \partial \Delta) \vee(\rho / \sigma) \stackrel{\left(i_{1}, i_{2}\right)}{\approx}(\Delta \cup \rho) / \sigma .
$$

Пространство $\Delta / \partial \Delta$ гомеоморфно букету всех пространств $d(p) / \partial d(p)$, и пространство $\rho / \sigma$ гомеоморфно (посредством отображения $(-v)_{[\lambda, a]}^{\curvearrowright}$ ) пространству $\left(\left(\partial_{0} W\right)^{\{\leqslant k\}} /\left(\partial_{0} W\right)^{\{\leqslant k-1\}}\right)$. Итак, мы получили следующий гомеоморфизм:

$$
\begin{aligned}
R / S \approx\left(\left(\partial_{1} W\right)^{\{\leqslant k\}}\right. & \left./\left(\partial_{1} W\right)^{\{\leqslant k-1\}}\right) \vee \Sigma\left(\left(\partial_{1} W\right)^{\{\leqslant k-1\}} /\left(\partial_{1} W\right)^{\{\leqslant k-2\}}\right) \\
& \vee\left(\bigvee_{p \in S_{k}(\phi)} d(p) / \partial d(p)\right) \vee\left(\left(\partial_{0} W\right)^{\{\leqslant k\}} /\left(\partial_{0} W\right)^{\{\leqslant k-1\}}\right) .
\end{aligned}
$$

Гомологии этого букета изоморфны

$$
C_{k}\left(u_{1}\right) \oplus C_{k}\left(u_{0}\right) \oplus C_{k}(v) \oplus C_{k-1}\left(u_{1}\right) .
$$

Ясно, что образы прямых слагаемых этих групп в $H_{k}\left(W^{\langle k\rangle}, W^{\langle k-1\rangle}\right)$ равны $\operatorname{Im} \mathscr{J}_{1}$, $\operatorname{Im} \mathscr{J}_{0}, \operatorname{Im} \mathscr{I}, \operatorname{Im} \mathscr{S}$ соответственно.

ЗАмЕчАнИЕ 5.8. В качестве побочного продукта нашего доказательства получаем следуюшее: включение

$$
\left.(\Delta / \partial \Delta) \vee(\rho / \sigma) \stackrel{j}{\hookrightarrow} W^{\langle k\rangle}\right|_{[\lambda, \mu]} /\left.W^{\langle k-1\rangle}\right|_{[\lambda, \mu]}
$$

является гомологической эквивалентностью и, следовательно, индуцирует изоморфизм

$$
J: C_{*}(v) \oplus C_{*}\left(u_{0}\right) \stackrel{\approx}{\longrightarrow} H_{*}\left(\left.W^{\langle k\rangle}\right|_{[\lambda, \mu]},\left.W^{\langle k-1\rangle}\right|_{[\lambda, \mu]}\right) .
$$

5.3. Граничные операторы, ассоциированные с фильтрациями морсовского типа. В предыдушем пункте мы видели, что $W^{\langle k\rangle}$ образует клеточную фильтрацию $W$. Поскольку $W^{\langle k\rangle}=\varnothing$ при $k<0$, то гомология $W$ может быть вычислена исходя из цепного комплекса, ассоциированного с фильтрацией. Именно, положим $E_{k}=H_{k}\left(W^{\langle k\rangle}, W^{\langle k-1\rangle}\right)$. Предложение 1.3 из [17; гл. 5] утверждает, что гомологии $H_{*}(W)$ изоморфны гомологиям комплекса $\left(E_{*}, d_{*}\right)$, где $d_{k}: E_{k} \rightarrow E_{k-1}-$ граничный оператор точной последовательности тройки $\left(W^{\langle k\rangle}, W^{\langle k-1\rangle}, W^{\langle k-2\rangle}\right)$. В этом пункте мы вычислим матрицу этого граничного оператора по отношению к разложению $E_{*}$ в прямую сумму, полученному в теореме 5.5 . Положим $d_{s}^{\prime}=L_{s}^{-1} \circ D_{s} \circ L_{s+1}$. Тогда $d_{s}^{\prime}$ задается $(4 \times 4)$-матрищей гомоморфизмов групп. Чтобы описать элементы этой матрицы, напомним, что $C_{*}\left(u_{1}\right), C_{*}\left(u_{0}\right), C_{*}(v)$ сами являются цепньми комплексами, и обозначим соответствуюшие граничные операторы посредством $\partial_{*}^{(1)}, \partial_{*}^{(0)}, \partial_{*}$. 
ПРЕДЛОЖЕНИЕ 5.9. Матрища для $d_{k+1}^{\prime}$ ecmb

$$
\left(\begin{array}{cccc}
\partial_{k+1}^{(1)} & 0 & 0 & \mathrm{Id} \\
0 & \partial_{k+1}^{(0)} & P_{k} & -\mathscr{H}_{k}(-v) \\
0 & 0 & \partial_{k+1} & -N_{k} \\
0 & 0 & 0 & -\partial_{k}^{(1)}
\end{array}\right)
$$

Здесь $\mathscr{H}_{k}(-v)$ - оператор гомологического градиентного спуска (см. теорему 4.8), $P_{k}, N_{k}$ - некоторые гомоморфизмы, которые будут определены в доказательстве.

ДокАЗАТЕльство. В доказательстве будем использовать обозначения и определения из пा. 5.1 и 5.2.

Первые два столбца. Они получаются прямо из определения $\mathscr{J}_{0}, \mathscr{J}_{1}$ : достаточно применить функториальность граничных операторов, ассоциированных с фильтрациями.

Третий столбец. Из леммы 5.7 следует, что достаточно вычислить класс гомологий элемента $\partial d(p)$ в $\left(\left.W^{\langle k\rangle}\right|_{[\lambda, b]},\left.W^{\langle k-1\rangle}\right|_{[\lambda, b]}\right)$. Поскольку $\partial d(p)$ принадлежит $\phi^{-1}(\mu)$, достаточно выгислить класс гомологий $\partial d(p)$ в $\left(\left.W^{\langle k\rangle}\right|_{[\lambda, \mu]},\left.W^{\langle k-1\rangle}\right|_{[\lambda, \mu]}\right)$. Гомологии этой пары изоморфны $C_{k}(v) \oplus C_{k}\left(u_{0}\right)$ (согласно замечанию 5.8). Легко убедиться, что проекция $[\partial d(p)]$ на первую компоненту прямой суммы равна $\partial_{k+1}([p])$. Символом $P_{k}$ обозначим гомоморфизм проекции на вторую компоненту. На этом вычисление третьего столбца заканчивается.

Гомоморфизм $N_{k}$. Пусть $x \in C_{k}\left(u_{1}\right)=H_{*}\left(\left(\partial_{1} W\right)^{\{\leqslant k\}},\left(\partial_{1} W\right)^{\{\leqslant k-1\}}\right)$.

Выберем представляюшую $x$ сингулярную цепь $\bar{x}$. Из условия (ㄷ) вытекает, что $(-v)$ спускает $\bar{x}$ до $\phi^{-1}(\mu)$; рассмотрим спушенную сингулярную цепь $\overline{\bar{x}}$ как цикл в паре

$$
\left(\left.W^{\langle k\rangle}\right|_{[\lambda, \mu]},\left.W^{\langle k-1\rangle}\right|_{[\lambda, \mu]}\right) .
$$

Символом $N_{k}(x)$ обозначим проекцию элемента $J^{-1}([x])$ на прямое слагаемое $C_{*}(v)$ группы $C_{*}(v) \oplus C_{*}\left(u_{0}\right)$ (см. формулу $\left.(28)\right)$.

Напомним, что $C_{k}(v)$ - свободная абелева группа, порожденная элементами $[p]$, $p \in S_{k}(\phi)$. Запишем $N_{k}(x)=\sum_{p \in S_{k}(\phi)}\langle x, p\rangle[p]$. Тогда отображение $x \mapsto\langle x, p\rangle$ является гомоморфизмом $C_{k}\left(u_{1}\right) \rightarrow \mathbb{Z}$, которьй нам пригодится в дальнейшем. Теперь мы дадим интерпретацию числа $\langle x, p\rangle$ в случае, когда выполнены следующие ограничения на $x$ :

$x$ представлен ориентированным подмногообразием $X$ многообразия $\partial_{1} W$, принадлежашим $\operatorname{Int}\left(\partial_{1} W\right)^{\{\leqslant k\}}$ и трансверсальным к $D(p,-v) \cap \partial_{1} W$.

Рассмотрим коориентированное $(n-k-1)$-мерное подмногообразие $B_{p}=D(p,-v) \cap$ $\partial_{1} W$ многообразия $\partial_{1} W$. Из условия (ㄷ) вытекает, что $B_{p} \subset \phi_{1}^{-1}\left(\left[\alpha_{k}, \alpha_{n}\right]\right)$. Имеем следуюшую формулу для алгебраического индекса пересечения $X$ с $B_{p}$ :

$$
\langle x, p\rangle=X \# B_{p}
$$

Итак,

$$
N_{k}(x)=\sum_{p \in S_{k}(\phi)}\left(X \# B_{p}\right)[p] .
$$


Четвертыи столбеи. Пусть $x \in C_{k}\left(u_{1}\right)=H_{k}\left(\left(\partial_{1} W\right)^{\{\leqslant k\}},\left(\partial_{1} W\right)^{\{\leqslant k-1\}}\right)$. Чтобы упростить обозначения, предположим, что $x$ представлен ориентированным подмногообразием $X$ многообразия $\partial_{1} W, \operatorname{dim} X=k$, таким образом, что $X \subset\left(\partial_{1} W\right)^{\{\leqslant k\}}$, $\partial X \subset\left(\partial_{1} W\right)^{\{\leqslant k-1\}}$. Тогда из определения $\mathscr{S}(x)\left(\right.$ см. п. 5.2) следует, что $d_{k+1}(\mathscr{S}(x))$ представляется суммой трех ориентированных особых многообразий с границей:

$$
X-G_{k}\left(\partial X \times\left[a_{k+1}, b\right]\right)-G_{k}\left(X \times\left\{a_{k+1}\right\}\right) .
$$

Первый член соответствует верхнему члену четвертого столбца. Второй и третий члены (32) не являются циклами пары $\left(W^{\langle k\rangle}, W^{\langle k-1\rangle}\right)$. Это можно поправить следующим образом. Каждая $(-v)$-траектория, начинающаяся в $\partial X$, достигает $\phi^{-1}\left(a_{k}\right)$, и мы можем записать

$$
G_{k}\left(\partial X \times\left[a_{k+1}, b\right]\right)=G_{k}\left(\partial X \times\left[a_{k}, b\right]\right)-G_{k}\left(\partial X \times\left[a_{k}, a_{k+1}\right]\right),
$$

так что тогда $d_{k+}(\mathscr{S}(x))$ представляется суммой

$$
X-G_{k}\left(\partial X \times\left[a_{k}, b\right]\right)+Y,
$$

где $Y=G_{k}\left(\partial X \times\left[a_{k}, a_{k+1}\right]\right)-G_{k}\left(\partial X \times\left\{a_{k+1}\right\}\right)$. Теперь два последних члена в правой части (34) являются циклами в $\left(W^{\langle k\rangle}, W^{\langle k-1\rangle}\right)$. Гомологический класс $G_{k}\left(\partial X \times\left[a_{k}, b\right]\right)$ очевидно совпадает с $\mathscr{S}\left(\partial_{k}^{(1)} x\right)$. Несложно проверить, что $\mathscr{L}_{k}^{-1}(Y)$ совпадает с элементом $-N_{k}(x)-\mathscr{H}_{k}(-v)(x)$, на чем и заканчивается вычисление четвертого столбца.

5.4. Фильтрации морсовского типа регулярных накрытий кобордизмов. В предыдушем пункте мы видели, что возможно вычислить гомологии $H_{*}(W)$ исходя из цепного комплекса $E_{*}$, ассоциированного с фильтрацией $W^{\langle k\rangle}$. В этом пункте мы усилим этот результат и покажем, как восстановить простой гомотопический тип многообразия $W$.

Пусть $p: \widetilde{W} \rightarrow W$ - регулярное накрытие $W$ со структурной группой $H$. Для $A \subset W$ мы обозначим $p^{-1}(A)$ посредством $\widetilde{A}$.

Фильтрация морсовского типа $W^{\langle k\rangle}$, построенная в предыдушем пункте, позволяет определить фильтрацию $\widetilde{W}^{\langle k\rangle}=p^{-1}\left(W^{\langle k\rangle}\right)$ многообразия $\widetilde{W}$. Прямое обобшение результатов предыдущего пункта приводит к следующему утверждению, описьвающему структуру относительных гомологий $H_{*}\left(\widetilde{W}^{\langle k\rangle}, \widetilde{W}^{\langle k-1\rangle}\right)$.

ПРЕДЛОЖЕНИЕ 5.10.

1. $H_{s}\left(\widetilde{W}^{\langle k\rangle}, \widetilde{W}^{\langle k-1\rangle}\right)=0$, еслu $s \neq k$.

2. Положим $\widetilde{E}_{k}=H_{k}\left(\widetilde{W}^{\langle k\rangle}, \widetilde{W}^{\langle k-1\rangle}\right)$. Тогда $\widetilde{E}_{k}-$ свободный $\mathbb{Z} H$-модуль и существует изоморфизм $\mathbb{Z} H$-модулей

$$
\widetilde{L}_{k}: \widetilde{C}_{k}\left(u_{1}\right) \oplus \widetilde{C}_{k}\left(u_{0}\right) \oplus \widetilde{C}_{k}(v) \oplus \widetilde{C}_{k-1}\left(u_{1}\right) \rightarrow \widetilde{E}_{k} .
$$

3. Пусть $\widetilde{d}_{k+1}: \widetilde{E}_{k+1} \rightarrow \widetilde{E}_{k}-$ граничный оператор точной последовательности тройки $\left(\widetilde{\widetilde{W}}^{\langle k+1\rangle}, \widetilde{W}^{\langle k\rangle}, \widetilde{W}^{\langle k-1\rangle}\right)$. Тогда матричей гомоморфизма $\widetilde{d}_{k+1}^{\prime}=\left(\widetilde{L}_{k}\right)^{-1} \circ \widetilde{d}_{k+1} \circ \widetilde{L}_{k+1}$ является

$$
\left(\begin{array}{cccc}
\widetilde{\partial}_{k+1}^{(1)} & 0 & 0 & \text { Id } \\
0 & \widetilde{\partial}_{k+1}^{(0)} & \widetilde{P}_{k} & -\widetilde{\mathscr{H}}_{k}(-v) \\
0 & 0 & \widetilde{\partial}_{k+1} & -\widetilde{N}_{k} \\
0 & 0 & 0 & -\widetilde{\partial}_{k}^{(1)}
\end{array}\right) .
$$


$\left(\right.$ Здесь $\widetilde{\mathscr{H}}_{k}(-v): \widetilde{C}_{k}\left(u_{1}\right) \rightarrow \widetilde{C}_{k}\left(u_{0}\right)$ - оператор гомологического градиентного спуска, ассоциированный с накрытием $\widetilde{W} \rightarrow W$, см. п. 4.4.)

ДокАЗАтЕльство. Это предложение доказьвается так же, как предложение 5.9. Мы остановимся только на тех моментах доказательства, которые существенны для дальнейшего, а именно, мы изложим построение цепей, представляюших образуюшие модулей $\widetilde{E}_{k}$, а также построение гомоморфизма $\widetilde{N}_{k}$.

Образующие модуля $\widetilde{E}_{k}$. Прежде всего, выберем для каждой точки $x \in S(\phi) \cup$ $S\left(\phi_{0}\right) \cup S\left(\phi_{1}\right)$ некоторое ее поднятие $\widetilde{x}$ в накрытие $\widetilde{W}$. Тогда для каждого $r \in S_{k}(\phi)$ определено поднятие $d(\tilde{r})$ диска $d(r)$ в $W$. Ясно, что $d(\tilde{r}) \subset \widetilde{W}^{\langle k\rangle}$, $\partial d(\tilde{r}) \subset \widetilde{W}^{\langle k-1\rangle}$. Определим $\widetilde{L}_{k}([\tilde{r}])$ как образ фундаментального класса пары $(d(\tilde{r}), \partial d(\tilde{r}))$ в $H_{*}\left(\widetilde{W}^{\langle k\rangle}, \widetilde{W}^{\langle k-1\rangle}\right)$.

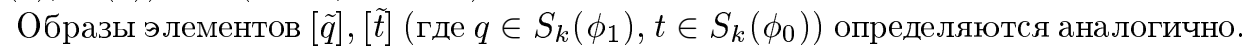

Переходя к образуюшим четвертого прямого слагаемого, отметим, что поднятие $\tilde{q}$ определяет поднятие $T_{k}$ многообразия $G_{k}\left(d(q) \times I_{k}\right)$ в $\widetilde{W}$. Пространство $T_{k}$ является ориентированным топологическим многообразием с краем, так что определен его фундаментальный класс в $H_{k}\left(T_{k}, \partial T_{k}\right)$. Образ этого фундаментального класса в $H_{k}\left(\widetilde{W}^{\langle k\rangle}, \widetilde{W}^{\langle k-1\rangle}\right)$ обозначим символом $[[\tilde{q}]]$ и определим $\widetilde{L}_{k}$ на компоненте $\widetilde{C}_{k-1}\left(u_{1}\right)$ формулой $\widetilde{L}_{k}([\tilde{q}])=[[\tilde{q}]]$.

Гомоморфизм $\widetilde{N}_{k}$. Пусть $x \in \widetilde{C}_{k}\left(u_{1}\right)=H_{k}\left(\left(\partial_{1} \widetilde{W}\right)^{\{\leqslant k\}},\left(\partial_{1} \widetilde{W}\right)^{\{\leqslant k-1\}}\right)$. Выберем сингулярную цепь $\bar{x}$, представляющую $x$. Из условия $(\mathfrak{C})$ вытекает, что $(-v)$ спускает $\bar{x}$ до $\phi^{-1}\left(a_{k+1}\right)^{\sim}$. Результат спуска - сингулярная цепь $\overline{\bar{x}}$ - есть цикл в паре

$$
\left(\left.\widetilde{W}^{\langle k\rangle}\right|_{[\lambda, \mu]},\left.\widetilde{W}^{\langle k-1\rangle}\right|_{[\lambda, \mu]}\right) .
$$

Аналогично лемме 5.7, включение

$$
\left(\left(\left.\Delta \cup Y_{k+1}\right|_{a_{k}}\right)^{\sim},\left.\widetilde{Y}_{k}\right|_{a_{k}}\right) \hookrightarrow\left(\left.\widetilde{W}^{\langle k\rangle}\right|_{[\lambda, \mu]},\left.\widetilde{W}^{\langle k-1\rangle}\right|_{[\lambda, \mu]}\right)
$$

индуцирует изоморфизм в гомологиях

$$
\widetilde{J}: \widetilde{C}_{k}(v) \oplus \widetilde{C}_{k-1}\left(u_{0}\right) \rightarrow H_{*}\left(\left.\widetilde{W}^{\langle k\rangle}\right|_{[\lambda, \mu]},\left.\widetilde{W}^{\langle k-1\rangle}\right|_{[\lambda, \mu]}\right)
$$

Символом $\widetilde{N}_{k}(x)$ обозначим проекцию элемента $\widetilde{J}^{-1}([\bar{x}])$ на первое слагаемое. Мы имеем эквивариантньй аналог формул (30) и (31). Именно, для каждого $p \in S_{k}(\phi)$ выберем поднятие $\tilde{p} \in \widetilde{M}$. Напомним, что гомологические классы $[\tilde{p}]$ пар $(d(\tilde{p}), \partial d(\tilde{p}))$ образуют свободный базис модуля $\widetilde{C}_{k}(v)$. Запишем

$$
\widetilde{N}_{k}(x)=\sum_{p \in S_{k}(\phi)}[\tilde{p}]\langle\langle x, p\rangle\rangle,
$$

где $\langle\langle x, p\rangle\rangle \in \mathbb{Z} H$; тогда отображение $x \rightarrow\langle\langle x, p\rangle\rangle$ является гомоморфизмом $\mathbb{Z} H$-модулей $\widetilde{C}_{k}\left(u_{1}\right) \rightarrow \mathbb{Z} H$. Предположим теперь, что $x$ есть фундаментальньй класс поднятия $\widetilde{X}$ в $\partial_{1} \widetilde{W}$ некоторого ориентированного многообразия $X \subset \partial_{1} W$, трансверсального к $B_{p}=D(p,-v) \cap \partial_{1} W$. Имеется поднятие $\widetilde{B}_{p}$ многообразия $B_{p} \subset \partial_{1} W$ в $\partial_{1} \widetilde{W}$, 
и, стало быть, для каждого $g \in H$ определен индекс пересечения $\widetilde{X} \# \widetilde{B}_{p} g \in \mathbb{Z}$. Выполняется следующее равенство:

$$
\langle\langle x, p\rangle\rangle=\sum_{g \in H}\left(\widetilde{X} \# \widetilde{B}_{p} g\right) g
$$

и, таким образом,

$$
\widetilde{N}_{k}(x)=\sum_{p \in S_{k}(\phi), g \in H}\left(\widetilde{X} \# \widetilde{B}_{p} g\right)[\tilde{p}] g
$$

Итак, цепной комплекс $\left\{\cdots \longrightarrow \widetilde{E}_{k} \stackrel{\widetilde{d}_{k}}{\longrightarrow} \widetilde{E}_{k-1} \longrightarrow \cdots\right\}$ является свободньгм $\mathbb{Z} H$-комплексом. Пункт 1 предложения 5.10 утверждает, что фильтрация $\widetilde{W}\langle k\rangle$ является клеточной, поэтому $H_{*}\left(\widetilde{E}_{*}\right) \approx H_{*}(\widetilde{W})$ (и, как несложно показать, этот изоморфизм можно выбрать так, чтобы он коммутировал с $\mathbb{Z} H$-действием).

Можно сказать и больше. Выберем $C^{1}$-триангулящию $\Delta$ многообразия $W$. Прообразы в $\widetilde{W}$ симплексов триангуляции $\Delta$ определяют $H$-инвариантную триангуляцию многообразия $\widetilde{W}$. Поэтому комплекс симплициальных цепей $C_{*}^{\Delta}(\widetilde{W})$ многообразия $\widetilde{W}$ является цепным комплексом свободных $\mathbb{Z} H$-модулей. Выбрав и зафиксировав поднятия в $\widetilde{W}$ симплексов триангуляции $\Delta$, получим базис в этом комплексе.

С другой стороны, выбрав ориентации нисходящих дисков и поднятия в $\widetilde{W}$ всех критических точек $\phi_{0}, \phi_{1}, \phi$, получим $\mathbb{Z} H$-базис в каждом из цепных комплексов $\widetilde{C}\left(u_{1}\right)$, $\widetilde{C}_{*}\left(u_{0}\right), \widetilde{C}_{*}(v)$. Изоморфизм $\widetilde{L}_{k}$ переносит этот базис в $\widetilde{E}_{*}$.

Чтобы сфформулировать следуюшее предложение, нам нужны два определения.

ОПРЕДЕЛЕНИЕ 5.11.

1. Пусть $R$ - кольцо. $R$-комплекс - это конечньй цепной комплекс конечно-порожденных свободных правых $R$-модулей с фиксированными базисами.

2. Два $\mathbb{Z} H$-комплекса $C_{*}, D_{*}$ назьваются просто гомотопически эквивалентными , если существует гомотопическая эквивалентность $\phi: C_{*} \rightarrow D_{*}$ такая, что кручение $\tau(\phi) \in W h(\mathbb{Z} H)$ равно нулю.

ПРЕДЛОЖЕНИЕ 5.12. $\mathbb{Z} H$-комплексы $C_{*}(\widetilde{W})$ и $\widetilde{E}_{*}$ являются просто гомотопически әквивалентными.

ДокАЗАТЕльСтво. Для замкнутых многообразий $(\partial W=\varnothing)$ это предложение доказано в [3; приложение, теорема А.5]. В общем случае доказательство аналогично. Мы приведем лишш идею доказательства. Выберем $C^{1}$-триангулящию в $W$ так, чтобы она удовлетворяла следуюшему условию $(\mathscr{T})$.

$(\mathscr{T}):$ Все подпространства $\partial_{0} W, \partial_{1} W$ являются симплициальными подкомплексами $W$. Все поверхности уровня $\phi^{-1}\left(a_{i}\right), \phi_{1}^{-1}\left(\alpha_{i}\right), \phi_{0}^{-1}\left(\beta_{i}\right)$ и диски $d(p), d(q), d(r)$, где $p \in S(\phi), q \in S\left(\phi_{0}\right), r \in S\left(\phi_{1}\right)$, являются симплициальным подкомплексами $W$. Для каждого $k$ подпространства $W^{\langle k\rangle}$ и подпространства $R, S$, определенные в (21) и (22), суть симплициальные подкомплексы $W$.

(Доказательство сушествования такой триангулящии представляет собой стандартное упражнение в теории триангуляции многообразий. В [3; с. 330-332] можно найти детальное доказательство аналогичного утверждения.) 
Далее мы будем предполагать, что читатель знаком со статьей [3]. Чтобы согласовать наши обозначения с обозначениями из $\S 3$ работы [3], положим $C_{*}=$ $C_{*}^{\Delta}(\widetilde{W}), C_{*}^{(k)}=C_{*}^{\Delta}\left(\widetilde{W}^{\langle k\rangle}\right)$; тогда $\mathbb{Z} H$-комплекс $\widetilde{E}_{*}$, определенный вьше, есть в точности комплекс $C_{*}^{g r}$ в обозначениях [3]. Из предложения 5.10 вытекает, что фильтрация $C_{*}^{(k)}$ комплекса $C_{*}$ является хорошей (nісе; определение хорошей фильтрации см. в [3; с. 310]).

Поэтому ([3; следствие 3.4$]$ ) существует каноническая цепная гомотопическая эквивалентность $\xi: C_{*}^{g r} \rightarrow C_{*}$. Гомотопический класс этой цепной гомотопической эквивалентности однозначно определяется следуюшими требованиями:

F1) $\xi\left(C_{k}^{g r}\right) \subset C_{*}^{(k)}$ для каждого $k$;

F2) гомоморфизм $C_{k}^{g r} \rightarrow H_{k}\left(C_{*}^{(k)}, C_{*}^{(k-1)}\right)$, индуцированный отображением $\xi$, совпадает с тождественным.

Определим $\mathbb{Z} G$-комплекс $\mu(k)_{*}$, положив

$$
\left\{\begin{array}{l}
\mu(k)_{j}=0, \quad \text { если } j \neq k, \\
\mu(k)_{k}=H_{k}\left(C_{*}^{(k)}, C_{*}^{(k-1)}\right) .
\end{array}\right.
$$

Цепное отображение $\xi$ определяет цепные гомотопические эквивалентности

$$
\xi_{k}: \mu(k)_{*} \rightarrow C_{*}^{(k)} / C_{*}^{(k-1)} .
$$

Нетрудно доказать по индукции, что если все отображения $\xi_{k}$ являются простыми гомотопическими эквивалентностями, то и само $\xi$ есть простая гомотопическая эквивалентность.

Остается показать, что каждое $\xi_{k}$-простая гомотопическая эквивалентность. Это мы сделаем в три шага.

Первый шаг: доказьваем, что включение $(R, S) \hookrightarrow\left(W^{\langle k\rangle}, W^{\langle k-1\rangle}\right)$ индуцирует простую гомотопическую эквивалентность $\mathbb{Z} H$-комплексов

$$
j: C_{*}(\widetilde{R}, \widetilde{S}) \rightarrow C_{*}\left(\widetilde{W}^{\langle k\rangle}, \widetilde{W}^{\langle k-1\rangle}\right)=C_{*}^{(k)} / C_{*}^{(k-1)}
$$

(доказательство такое же, как в лемме 5.7).

Второй шаг: отображение $\xi_{k}$ пропускается черезгомотопическую эквивалентность $\xi_{k}^{\prime}: \mu(k)_{*} \rightarrow C_{*}(\widetilde{R}, \widetilde{S})$. Гомологии $L_{*}=C_{*}(\widetilde{R}, \widetilde{S})$ равны нулю в размерностях, отличных от $k$, а модуль $H_{k}(\widetilde{R}, \widetilde{S}) \approx \widetilde{E}_{k}-$ свободньй. Итак, вводя тривиальную фильтрацию $\left\{0, L_{*}\right\}$ в $L_{*}$, мы получаем цепную гомотопическую эквивалентность $\xi_{k}^{\prime \prime}: \mu(k)_{*} \rightarrow L_{*}$ (см. [3; следствие 3.4$]$ ), и несложно проверить, что $\xi_{k}^{\prime \prime}$ гомотопно $\xi_{k}^{\prime}$.

Третий шаг: можно показать, что $\xi_{k}^{\prime \prime}$ расшепляется в сумму четырех гомотопических эквивалентностей, соответствуюших расшеплениям $R / S$ в букет четырех слагаемых в (26). Можно показать, что каждая их этих эквивалентностей является простой гомотопической эквивалентностью. 


\section{§6. Алгебраические леммы}

Здесь мы будем пользоваться обозначениями из п. 1.2 и введем несколько новых. Скажем, что $\phi$ есть $U$-простая гомотопическая әквивалентность, если $\tau(\phi \mid U)$ равно нулю. Если значение $U$ ясно из контекста, то вместо словосочетания $U$-простая гомотопическая эквивалентность мы говорим простая гомотопическая эквивалентность. Отметим, что если каждое $\phi_{i}: F_{i} \rightarrow D_{i}$ является изоморфизмом, то $\tau(\phi)=\sum_{i}(-1)^{i}\left[\phi_{i}\right] .^{5}$

6.1. Лемма о конусоподобных цепных комплексах. Пусть $C_{*}-R$-комплекс, и пусть $d_{k}: C_{k} \rightarrow C_{k-1}$ - его граничные гомоморфизмы. Положим $E_{k}=C_{k} \oplus C_{k-1}$. Пусть для каждого $k$ заданы изоморфизм $A_{k}: C_{k} \rightarrow C_{k}$ игомоморфизм $d_{k}^{\prime}: C_{k} \rightarrow C_{k-1}$. Пусть $\mathscr{D}_{k}: E_{k} \rightarrow E_{k-1}$ - гомоморфизм с матрицей $\left(\begin{array}{cc}d_{k} & A_{k-1} \\ 0 & d_{k-1}^{\prime}\end{array}\right)$.

Предположим, что $\mathscr{D}_{k-1} \circ \mathscr{D}_{k}=0$, так что $\left(E_{*}, \mathscr{D}_{*}\right)$ является цепньм комплексом.

Лемма 6.1.

1. Комплекс $E_{*}$ гомотопически әквивалентен нулю.

2. $\tau\left(E_{*}\right)=\sum_{i}(-1)^{i+1}\left[A_{i}\right]$.

ДоказАтельство. Рассмотрим конус $L_{*}$ тождественного отображения Id: $C_{*} \rightarrow$ $C_{*}$; мы имеем: $L_{k}=C_{k} \oplus C_{k-1}$, и матрицей граничного оператора $\Delta_{k}: L_{k} \rightarrow L_{k-1}$ является $\left(\begin{array}{cc}d_{k} & \mathrm{Id} \\ 0 & -d_{k-1}\end{array}\right)$.

Ясно, что $\tau\left(L_{*}\right)=0$. Мы знаем, что $\left(E, \mathscr{D}_{*}\right)$ - цепной комплекс, а это эквивалентно вьполнению следуюших условий:

1. $d_{k}^{\prime}$ - граничньй оператор;

2. $d_{k} A_{k}+A_{k-1} d_{k}^{\prime}=0$ для каждого $k$.

Определим отображение $\lambda: E_{*} \rightarrow L_{*}$ следуюшим образом: $\left(c_{k}, c_{k-1}\right) \mapsto\left(c_{k}\right.$, $\left.A_{k-1} c_{k-1}\right)$. Простое вычисление показывает, что $\lambda$ - изоморфизм комплексов. Поэтому $E_{*}$ гомотопически эквивалентен нулю и $\tau\left(L_{*}\right)=\tau\left(E_{*}\right)+\tau(\lambda)=\tau(\lambda)$. Но $\tau(\lambda)=\sum_{i}(-1)^{i+1}\left[A_{i}\right]$.

6.2. Кольцо $A[t]$. Пусть $A$ - произвольное коммутативное кольцо с единицей. Положим $R=A[t]$. Рассмотрим вложение $j: A \hookrightarrow A[t]$ и проекцию $\pi: A[t] \rightarrow A[t] / t A[t]$ $=A$. Тогда $\pi j=\mathrm{id}$. Если $C_{*}-R$-комплекс, то комплекс $C_{*}^{-}=C_{*} \underset{A}{\otimes} R$ называется $R$-расширением комплекса $C_{*}$ (или попросту расширением, если это не приводит к путанище). ${ }^{6}$ Пусть $f: C_{*} \rightarrow D_{*}$ - отображение $A$-комплексов. Отображение $f \otimes \mathrm{id}: C_{*} \otimes R \rightarrow D_{*} \otimes R$ назьвается $R$-расширением отображения $f$. Мы будем обозначать $R$-расширение отображения $f$ тем же символом $f$, если это не приводит к путанище. Отметим, что $C_{*}=C_{*}^{-} / t C_{*}^{-}$, так что $H_{*}\left(C_{*}^{-}\right)=0$, если и только если $H_{*}\left(C_{*}\right)=0$. Пусть $U-$ подгруппа группы $A^{\bullet}$. Отображение $j_{*}: K_{1}(A \mid U) \rightarrow$ $K_{1}(R \mid U)$ является мономорфизмом на прямое слагаемое; правым обратным к этому отображению является $\pi_{*}: K_{1}(R \mid U) \rightarrow K_{1}(A \mid U)$. Следующая лемма очевидна.

\footnotetext{
${ }^{5}$ Здесь мы придерживаемся соглашения о знаках, принятого в [4], так что нашекручение $\tau(C *)$ отличается по знаку от кручения из [18].

${ }^{6}$ Почему мы выбрали обозначение $C_{*}^{-}$, читатель поймет в следующем параграфе (см. $\left.(50)\right)$.
} 
ЛЕМма 6.2. Комплекс $C_{*}^{-}$является $U$-просто гомотопически әквивалентнымм нулю тогда и только тогда, когда комплекс $C_{*}$ является $U$-просто гомотопически эквивалентным нулю.

СЛЕДСТВИЕ 6.3. Пусть

$$
0 \longrightarrow M_{*} \stackrel{\phi}{\longrightarrow} N_{*} \longrightarrow P_{*} \longrightarrow 0
$$

- точная последовательность $R$-комплексов такая, что $P_{*}$ является $R$-расширением.

Отобрахсение $\phi$ является $U$-простой гомотопической әквивалентностью тогда и только тогда, когда ф/t является $U$-простой гомотопической эквивалентностью.

ДокАЗАТЕльство. Точная последовательность (42) расщепляется как точная последовательность $R$-модулей, следовательно, она остается точной и после тензорного умножения на $R / t R$. Далее, гомологии комплекса $P_{*}$ равны нулю тогда и только тогда, когда равны нулю гомологии комплекса $P_{*} / t P_{*}$, и, таким образом, $\phi / t$ является гомотопической эквивалентностью в том и только том случае, когда $\phi-$ гомотопическая эквивалентность. Далее, кручение отображения $\phi$ (соответственно, отображения $\phi / t$ ) равно кручению комплекса $P_{*}$ (соответственно, комплекса $\left.P_{*} / t P_{*}\right)$. Но $\tau\left(P_{*}\right)=j_{*}\left(\tau\left(P_{*} / t P_{*}\right)\right)$, ибо $P_{*}$ есть $R$-расширение.

\section{$\S$ 7. Условие $(\mathfrak{C} \mathscr{C})$, фильтрации морсовского типа для морсовских отображений $M \rightarrow S^{1}$ и рациональность новиковских коэффициентов инцидентности в случае $C^{0}$-общего положения}

7.1. Условие $(\mathfrak{C} \mathscr{C})$ и рациональность новиковских коэффициентов инцидентности в случае $C^{0}$-общего положения. В этом параграфе мы перейдем наконец к морсовским отображениям $M \stackrel{f}{\longrightarrow} S^{1}=\mathbb{R} / \mathbb{Z}$. Мы будем использовать определения и обозначения из п. 2.4.

Предположим, что $f$ примитивно, т.е. $f_{*}: H_{1}(M) \rightarrow H_{1}\left(S^{1}\right)=\mathbb{Z}$ - эпиморфио. Для упрошения записи предположим, что $1 \in S^{1}$ - регулярное значение функции $f$. Обозначим $f^{-1}(1)$ символом $V$. Напомним, что $\mathscr{C}: \bar{M} \rightarrow M$ - бесконечное циклическое накрытие, ассоциированное с $f$, и $F: \bar{M} \rightarrow \mathbb{R}$ - поднятие функции $f$. Положим $V_{\alpha}=F^{-1}(\alpha), W=F^{-1}([0,1])$ и $\left.\left.V^{-}=F^{-1}(]-\infty, 1\right]\right)$. Кобордизм $W$ есть результат разрезания $M$ вдоль $V$. Образующая $t$ структурной групшы накрытия $\mathscr{C}$ выбрана так, что $V_{\alpha} t=V_{\alpha-1}$. Положим $W_{s}=W t^{s}$; тогда $\bar{M}$ есть объединение $\bigcup_{s \in \mathbb{Z}} W_{s}$, причем $W$ пересекаются по многообразию $V_{-s}$.

Для каждого $k \in \mathbb{Z}$ ограничение $\mathscr{C}$ на $V_{k}$ является диффеоморфизмом $V_{k} \rightarrow V$. Введем в $M$ произвольную риманову метрику и поднимем ее до $t$-инвариантной римановой метрики на $\bar{M}$. Теперь $W$ - риманов кобордизм и, более того, - циклический кобордизм по отношению к изометрии $t^{-1}: \partial_{0} W=V_{0} \rightarrow \partial_{1} W=V_{1}$. Будем говорить, что $v$ удовлетворяет условию $(\mathfrak{C} \mathscr{C})(\mathfrak{C}$ - от слова клеточный (cellular) и $\mathscr{C}-$ от слова круг (circle)), если $(F \mid W)$-градиент $v$ удовлетворяет условию (C્Y) из п. 4.5 .

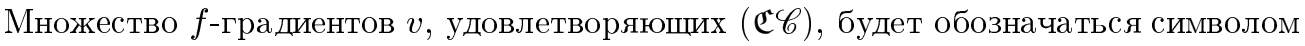


$\mathscr{G} \mathscr{C}(f)$. Из теоремы 4.12 следует, что множество $\mathscr{G} \mathfrak{C} \mathscr{C} \mathscr{T}(f)=\mathscr{G} \mathfrak{C}(f) \cap \mathscr{G} \mathscr{T}(f)$ является открытым и плотным подмножеством множества $\mathscr{G} \mathscr{T}(f)$ в $C^{0}$-топологии.

Пусть $v \in \mathscr{G} \mathfrak{C} \mathscr{C}(f)$. Напомним, что в условии (당 фигурирует упорядоченная функция Морса $\phi_{1}$ на $V_{1}$ и ее градиент $u_{1}$, а также упорядоченная функция Морса $\phi_{0}=\phi_{1} \circ t^{-1}$ на $V_{0}$ и ее градиент $u_{0}=(t)_{*}\left(u_{1}\right)$. Определим упорядоченную функцию Морса $\phi_{k}=\phi_{0} \circ t^{k}: V_{k} \rightarrow \mathbb{R}$ и $\phi_{k}$-градиент $u_{k}=\left(t^{-k}\right)_{*}\left(u_{0}\right)$.

ТеОРема 7.1. Пусть $v \in \mathscr{G} \mathfrak{C} \mathscr{C} T(f)$. Тогда каждий новиковский коэффициент инцидентности является рациональной функиией своего аргумента $t$.

ДокаЗАтельство. Пусть $r, s \in S(f)$, ind $r=l+1=$ ind $s+1$. Можно считать, что поднятия $\bar{r}, \bar{s}$ критических точек $r, s$ в $\bar{M}$ лежат в $W$. Тогда $n_{k}(r, s ; v)=0$ при $k<0$.

Рассмотрим ориентированное подмногообразие $S_{\bar{r}}=D(\bar{r}, v) \cap V_{0}$ подмногообразия $V_{0}$. Из условия $(\mathfrak{C})$ вытекает, что $S_{\bar{r}} \subset\left(V_{0}\right)^{\{\leqslant l\}}$ и что множество $S_{\bar{r}} \backslash \operatorname{Int}\left(V_{0}^{\{\leqslant l-1\}}\right)$ компактно, так что определен фундаментальный класс $\left[S_{\bar{r}}\right]$ многообразия $S_{\bar{r}}$ в группе $H_{l}\left(V_{0}^{\{\leqslant l\}}, V_{0}^{\{\leqslant l-1\}}\right)$. Применяя утверждение 1 теоремы 4.8 , по индукции получаем, что для каждого $k$ подмногообразие $X_{k}=(-v)_{[0,-k]}^{\rightsquigarrow}\left(S_{\bar{r}}\right)$ многообразия $V_{-k}$ лежит в $V_{-k}^{\{\leqslant l\}}$ и что $X_{k} \backslash \operatorname{Int}\left(V_{-k}^{\{\leqslant l-1\}}\right)$ компактно. Более того, фундаментальный класс многообразия $X_{k}$ в $H_{*}\left(V_{-k}^{\{\leqslant l\}}, V_{-k}^{\{\leqslant l-1\}}\right)$ совпадает с $\mathscr{H}_{l}^{k}(-v)\left(\left[S_{\bar{r}}\right]\right)$.

Положим $Y_{k}=B_{\bar{s} t^{k+1}}=D\left(\bar{s} t^{k+1},-v\right) \cap V_{-k}$. Тогда $Y_{k}$ - коориентированное $(n-l-1)$-мерное подмногообразие многообразия $V_{-k}($ где $n=\operatorname{dim} M)$ и $Y_{k} \subset$ $\phi_{-k}^{-1}\left(\left[\alpha_{l}, \alpha_{n+1}\right]\right)$. Поскольку $v$ удовлетворяет условию трансверсальности, то $X_{k} \pitchfork$ $Y_{k}$ и $n_{k+1}(r, s ; v)=X_{k} \# Y_{k}$. Применяя формулу (30) к кобордизму $W_{k+1}$, получаем:

$$
n_{k+1}(r, s ; v)=\left\langle\left[X_{k}\right], \bar{s} t^{k+1}\right\rangle .
$$

Обозначим $t^{-1} \mathscr{H}_{l}(-v)$ символом $h$; тогда

$$
n_{k+1}(r, s ; v)=\left\langle h^{k}\left(\left[S_{\bar{r}}\right]\right) t^{k}, \bar{s} t^{k+1}\right\rangle=\left\langle h^{k}\left(\left[\bar{S}_{r}\right]\right), \bar{s} t\right\rangle .
$$

Стало быть, верна следуюшая формула:

$$
n(r, s ; v)=n_{0}(r, s ; v)+t \sum_{k} \xi\left(A^{k} x\right) t^{k},
$$

где $A=h, x=\left[S_{\bar{r}}\right], \xi=\langle\cdot, \bar{s} t\rangle$. Теперь рациональность функции $n(r, s ; v)$ вытекает из утверждения (2).

7.2. Новиковские коэффициенты инцидентности, ассоциированные с регулярньм накрытием. В настояшем разделе мы обобшим конструкцию комплекса Новикова и изучим свойства рациональности для соответствующих коэффициентов инцидентности. Мы будем использовать определения и обозначения из пा. 1.2 и 2.4.

Пусть $f: M \rightarrow S^{1}$ - не гомотопное нулю морсовское отображение, $\mathscr{P}: \widetilde{M} \rightarrow M-$ регулярное накрытие со структурной группой $G$. Как всегда, мы предполагаем, что 


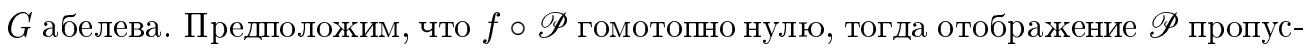
кается через $\mathscr{C}$ :

$$
\begin{gathered}
\widetilde{M} \stackrel{p}{\longrightarrow} \bar{M} \\
\mathscr{P} \searrow \quad \downarrow \mathscr{C} \\
\\
M
\end{gathered}
$$

Следовательно, существует поднятие функции $f: M \rightarrow S^{1}$ до функции Морса $\widetilde{F}: \widetilde{M} \rightarrow \mathbb{R}$. Гомоморфизм $f_{*}: \pi_{1} M \rightarrow \mathbb{Z}$ пропускается через гомоморфизм $\xi: G \rightarrow \mathbb{Z}$. Положим $\left.H=\operatorname{Ker} \xi, G_{-}=\xi^{-1}(]-\infty, 0\right]$ ). Выберем (как и раньше при построении первоначального варианта комплекса Новикова, см. п. 2.4) ориентации нисходяших дисков критических точек $f$. Выберем, кроме того, поднятие каждой критической точки $x$ функции $f$ в многообразие $\widetilde{M}$. Пусть $v \in \mathscr{G} \mathscr{T}(f)$. Поднимем $v$ до $G$-инвариантного векторного поля на $\widetilde{M}$ (которое будем обозначать той же буквой $v$ ). Пусть $x, y \in S(f)$, ind $x=$ ind $y+1$. Обозначим символом $\Gamma(x, y, g)$ множество $(-v)$-орбит, соединяющих $\widetilde{x}$ с $\widetilde{y} g$. Пусть $\gamma \in \Gamma(x, y, g)$. Тогда, как и ранее, определен знак $\varepsilon(\gamma) \in\{-1,1\}$. Положим

$$
\tilde{n}(x, y ; v)=\sum_{g \in G}\left(\sum_{\gamma \in \Gamma(x, y, g)} \varepsilon(\gamma) \cdot g\right) .
$$

Тогда $\tilde{n}(x, y ; v)$ - элемент $\widehat{\widehat{\Lambda}}$, и нетрудно показать, что $\tilde{n}(x, y ; v) \in \widehat{\Lambda}_{\xi}$. Элемент $\tilde{n}(x, y ; v)$ будет назьваться новиковским коэффициентом инцидентности (как и $n(x, y ; v))$. Пусть $\widetilde{\mathscr{C}}_{p}(v)$ - свободный правый $\widehat{\Lambda}_{\xi}$-модуль, натянутый на $S_{p}(f)$. Определим $\widehat{\Lambda}_{\xi}$-гомоморфизм $\widetilde{\mathscr{D}}_{p}: \widetilde{\mathscr{C}}_{p}(v) \rightarrow \widetilde{\mathscr{C}}_{p-1}(v)$, полагая

$$
\widetilde{\mathscr{D}}_{p}(x)=\sum_{y \in S_{p-1}(f)} y \cdot \tilde{n}(x, y ; v)
$$

Можно проверить, что $\widetilde{\mathscr{D}}_{p} \circ \widetilde{\mathscr{D}}_{p+1}=0$. Таким образом, мы получили свободный цепной комплекс $\widetilde{\mathscr{C}}_{*}(v)$ с фиксированным базисом, назьваемый комплексом Новикова. Более того, сушествует цепная гомотопическая эквивалентность $\phi: \widetilde{\mathscr{C}}_{*}(v) \rightarrow C_{*}^{\Delta}(\widetilde{M}) \underset{\Lambda}{\otimes} \widehat{\Lambda}_{\xi}$ такая, что образ $\tau(\phi)$ в $K_{1}\left(\widehat{\Lambda}_{\xi} \mid U_{\xi}\right)$ равен нулю ([3; теорема 2.2$\left.]\right)$.

Ввиду теоремы 7.1 естественно предположить, что если $v \in \mathscr{G} \mathfrak{C} \mathscr{T}(f)$, то новиковские коэффициенты инцидентности, соответствуюшие $v$, являются не просто числовыми рядами, а "рациональными функциями многих переменных". Точнее говоря, естественно ожидать, что $\tilde{n}(x, y ; v) \in \Lambda_{(\xi)}$. Это действительно так:

Теорема 7.2. Пусть $v \in \mathscr{G} \mathfrak{C} \mathscr{C} \mathscr{T}(f)$. Тогда все новиковские коэффициенты инцидентности лежат в $\Lambda_{(\xi)}$. 
ДокАЗАТЕльство этой теоремы происходит параллельно доказательству теоремы 7.1. Мы не будем приводить его полностью, а отметим только основные изменения, которые нужно сделать. Пусть $r, s \in S(f)$, ind $r=$ ind $s+1=l+1$. Можно считать, что все поднятия в $\widetilde{M}$ критических точек функции $f$ в $\widetilde{M}$ лежат в $\widetilde{W}$. Итак, мы получаем поднятие $X^{\prime}$ многообразия $X$ до $\widetilde{V}_{1}$. Для $a \in S(f)$ положим $\bar{a}=p(\widetilde{a})$. Выберем элемент $\theta \in G$ такой, что $\xi(\theta)=-1$. Каждая критическая точка функции $F: \bar{M} \rightarrow \mathbb{R}$ имеет вид $\bar{b} t^{k}$ с $k \in \mathbb{Z}, b \in S(f)$. Поднимем критическую точку $\bar{b} t^{k} \in \bar{M}$ до точки $\widetilde{b} \theta^{k} \in \widetilde{M}$. Тогда для каждого $k$ мы получаем поднятие $Y_{k}^{\prime}$ множества $Y_{k}$ в множество $\widetilde{V}_{-k} \subset \widetilde{M}$ такое, что $Y_{k}^{\prime}=Y_{0}^{\prime} \theta^{k}$. Как и в конще п. 4.5, определим эндоморфизм $\tilde{h}_{l}(-v)$ свободного правого $\mathbb{Z} H$-модуля $H_{*}\left(\widetilde{V}_{0}^{\{\leqslant l\}}, \widetilde{V}_{0}^{\{\leqslant l-1\}}\right)$ формулой $\tilde{h}_{l}(-v)=\widetilde{\mathscr{H}}_{l}(-v) \circ\left(\theta^{-1}\right)_{*}$. Так как мы рассматриваем только один $f$-градиент, будем писать $\tilde{h}_{s}$ вместо $\tilde{h}_{s}(-v)$.

Положим

$$
\tilde{n}_{k}(r, s ; v)=\sum_{g: \xi(g)=-k}\left(\sum_{\gamma \in \Gamma(r, s ; g)} \varepsilon(\gamma) g\right) .
$$

Тогда $\tilde{n}(r, s ; v)=\sum_{k \geqslant 0} \tilde{n}_{k}(r, s ; v)$ и выполнено следуюшее:

$$
\begin{aligned}
\tilde{n}_{k+1}(r, s ; v) & =\sum_{g \in H}\left(\widetilde{X}_{k} \# \widetilde{B}_{\widetilde{s} g \theta^{k+1}}\right) g \theta^{k+1} \\
& =\sum_{g \in H}\left(\widetilde{X}_{k} \theta^{-k} \# \widetilde{B}_{s} g \theta\right) g \theta^{k+1}=\left\langle\left\langle\left[\widetilde{X}_{k} \theta^{-k}\right], \widetilde{s} \theta\right\rangle\right\rangle \theta^{k+1}
\end{aligned}
$$

Применяя теорему 4.10, по индукции получаем, что $\left[\widetilde{X}_{k} \theta^{-k}\right]=\tilde{h}^{k}\left(\left[\widetilde{X}_{0}\right]\right)$. Итак,

$$
\tilde{n}(r, s ; v)=\tilde{n}_{0}(r, s ; v)+\sum_{k \geqslant 0}\left\langle\left\langle\tilde{h}^{k}\left(\left[\tilde{X}_{0}\right]\right), \widetilde{s} \theta\right\rangle\right\rangle \theta^{k+1}
$$

и этот последний элемент лежит в $\Lambda_{(\xi)}$ (чтобы доказать это, достаточно применить соответствуюшее обобщение утверждения (2)).

ЗАмЕчАниЕ 7.3. Приведем еше одну формулу для новиковских коэффициентов инцидентности, которая нам будет полезна в дальнейшем (она следует из (38)):

$$
\sum_{s} \tilde{n}_{k+1}(r, s ; v)[\widetilde{s}]=\widetilde{N}_{l}\left(\tilde{h}^{k}\left(\left[\widetilde{X}_{0}\right]\right) \theta^{k+1}\right.
$$

7.3. Фильтрации морсовского типа, построенные по морсовскому отображению $M \rightarrow S^{1}$. Мы будем использовать определения и обозначения пп. 7.1, 7.2. Пусть $v \in \mathscr{G} \mathfrak{C} \mathscr{C} \mathscr{T}(f)$.

Рассмотрим фильтрацию $W^{\langle k\rangle}$ кобордизма $W$, соответствуюшую векторному полю $v$, и введем $t$-инвариантую фильтрацию в множестве $V^{-}$, положив

$$
V_{\langle k\rangle}^{-}=\bigcup_{s \geqslant 0} t^{s} W^{\langle k\rangle} .
$$


Эта фильтрация в $V^{-}$индуцирует $G_{-}$-инвариантную фильтрацию $\widetilde{V}_{\langle k\rangle}^{-}$в множестве $\widetilde{V}^{-}$. В настоящем разделе мы будем заниматься восстановлением простого гомотопического типа $\mathbb{Z} G$-комплекса $C_{*}^{\Delta}(\widetilde{M})$ исходя из информации, содержашейся в последовательных факторах фильтрации $V_{\langle k\rangle}^{-}$. (В теории Морса, конечно, уже есть процедура такого рода: возьмем функцию Морса $g: M \rightarrow \mathbb{R}$ и построим соответствуюший комплекс Морса по $g$ и по накрытию $\mathscr{P}$. Процедура, которую мы собираемся предложить в этом разделе, имеет то преимушество, что она лучше приспособлена к новиковским гомологиям многообразия.) Следующее ниже предложение является естественным продолжением теоремы 5.5 и предложений 5.9 и 5.10 . Нам потребуется несколько определений. Положим

$$
\widetilde{C}_{*}^{-}\left(u_{1}\right)=\widetilde{C}_{*}\left(u_{1}\right) \underset{\mathbb{Z} H}{\otimes} \mathbb{Z} G_{-}, \quad \widetilde{C}_{*}^{-}(v)=\widetilde{C}_{*}(v) \underset{\mathbb{Z} H}{\otimes} \mathbb{Z} G_{-} .
$$

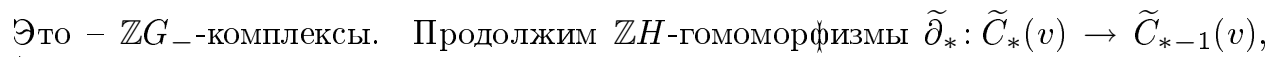

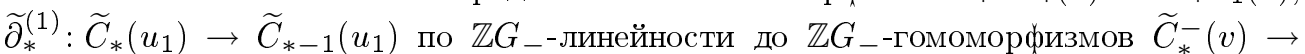
$\widetilde{C}_{*-1}^{-}(v)$, и, соответственно, $\widetilde{C}_{*}^{-}\left(u_{1}\right) \rightarrow \widetilde{C}_{*-1}^{-}\left(u_{1}\right)$. Продолженные гомоморфизмы будем обозначать теми же символами $\widetilde{\partial}_{*}^{(1)}, \widetilde{\partial}_{*}$. Выберем какой-нибудь элемент $\theta \in G_{(-1)}$. Имеется отождествление: $\mathbb{Z} G_{-}=\mathbb{Z} H[\theta]$. Отождествим $\mathbb{Z} H$-подмодуль $\widetilde{C}_{*}\left(u_{1}\right) \theta$ с $\widetilde{C}_{*}\left(u_{0}\right)$. Гомоморфизмы $\widetilde{\mathscr{H}}_{k}(-v): \widetilde{C}_{*}\left(u_{1}\right) \rightarrow \widetilde{C}_{*}\left(u_{0}\right)$ и $\widetilde{P}_{k}: \widetilde{C}_{*}(v) \rightarrow \widetilde{C}_{*}\left(u_{0}\right)$ можно рассматривать как гомоморфизмы со значениями в $\widetilde{C}_{*}^{-}\left(u_{1}\right)$. Продолжим их по $\mathbb{Z} G_{-}$-линейности на все $\widetilde{C}_{*}\left(u_{1}\right), \widetilde{C}_{*}(v)$ (сохраняя те же обозначения для продолжений). Положим $\tilde{h}_{k}=\mathscr{H}_{k}(-v) \theta^{-1}: \widetilde{C}_{*}\left(u_{1}\right) \rightarrow \widetilde{C}_{*}\left(u_{1}\right)$.

\section{ПРЕДЛОЖЕНИЕ 7.4 .}

1. $H_{s}\left(\widetilde{V}_{\langle k\rangle}^{-}, \widetilde{V}_{\langle k-1\rangle}^{-}\right)=0$, ecли $s \neq k$.

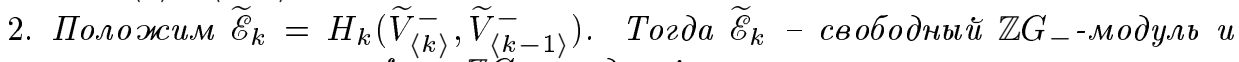

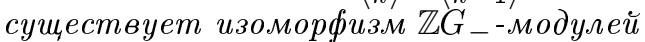

$$
\widetilde{\mathscr{L}}_{k}: \widetilde{C}_{k}^{-}\left(u_{1}\right) \oplus \widetilde{C}_{k}^{-}(v) \oplus \widetilde{C}_{k-1}^{-}\left(u_{1}\right) \stackrel{\approx}{\underset{\mathscr{E}}{\longrightarrow}} \widetilde{\mathscr{E}}_{k}
$$

3. Пусть $\widetilde{D}_{k+1}: \widetilde{\mathscr{E}}_{k+1} \rightarrow \widetilde{\mathscr{E}}_{k}-$ граничныц й оператор точной последовательности тройки $\left(\widetilde{V}_{\langle k+1\rangle}^{-}, \widetilde{V}_{\langle k\rangle}^{-}, \widetilde{V}_{\langle k-1\rangle}^{-}\right)$. Тогда матрица гомоморфизма $\widetilde{D}_{k+1}^{\prime}=\widetilde{\mathscr{L}}_{k}^{-1} \circ \widetilde{D}_{k+1} \circ \widetilde{\mathscr{L}}_{k+1}$ по отночению $к$ разложению $\widetilde{\mathscr{E}}_{k}$ в прямую суммму, указанному в (51), равна

$$
\left(\begin{array}{ccc}
\widetilde{\partial}_{k+1}^{(1)} & \widetilde{P}_{k+1} & \mathrm{Id}-\theta \tilde{h}_{k}(-v) \\
0 & \widetilde{\partial}_{k+1} & \widetilde{N}_{k} \\
0 & 0 & -\widetilde{\partial}_{k}^{(1)}
\end{array}\right)
$$


ДОКАЗАТЕЛЬСТВО. Мы рассмотрим лишш частньй случай $\widetilde{M}=\bar{M}$ (тогда $p$ является тождественным отображением). Общий случай отличается от этого частного случая в основном усложнением в обозначениях (надо добавить тильды и заменить $t$ на $\theta$ ).

Рассмотрим фильтрацию $W \prec k \succ$ кобордизма $W$, введенную в формуле (10). Положим $V_{\prec k \succ}^{-}=\bigcup_{s \geqslant 0} t^{s} W^{\prec k \succ}$. Несложно показать, что включения

$$
\left(V_{\prec k \succ}^{-}, V_{\prec k-1 \succ}^{-}\right) \hookrightarrow\left(V_{\prec k \succ}^{-}, V_{\langle k-1\rangle}^{-}\right) \hookrightarrow\left(V_{\langle k\rangle}^{-}, V_{\langle k-1\rangle}^{-}\right)
$$

являются гомотопическими эквивалентностями (это доказывается так же, как лемма 5.3). Рассмотрим подпространство

$$
W^{[k]}=\left(\partial_{1} W\right)^{\{\leqslant k\}} \cup Y_{k} \cup Z_{k}=W^{\prec k \succ} \backslash\left(\left(\partial_{0} W\right)^{\{\leqslant k\}} \backslash\left(\partial_{0} W\right)^{\{\leqslant k-1\}}\right)
$$

кобордизма $W$. Очевидно, что

$$
W^{\langle k-1\rangle} \subset W^{[k]} \subset W^{\prec k \succ},
$$

поэтому сушествует вложение пар

$$
\left(W^{[k]}, W^{\langle k-1\rangle}\right) \stackrel{\lambda}{\hookrightarrow}\left(V_{\prec k \succ}^{-}, V_{\langle k-1\rangle}^{-}\right) .
$$

Ясно, что

$$
\begin{gathered}
H_{k}\left(W^{[k]}, W^{\langle k-1\rangle}\right) \approx C_{k}\left(u_{1}\right) \oplus C_{k}(v) \oplus C_{k-1}\left(u_{1}\right), \\
H_{*}\left(W^{[k]}, W^{\langle k-1\rangle}\right)=0, \text { если } * \neq k .
\end{gathered}
$$

ЛЕмма 7.5. Индуиированный включением $\lambda$ гомоморфизм

$$
H_{*}\left(W^{[k]}, W^{\langle k-1\rangle}\right) \stackrel{\lambda_{*}}{\longrightarrow} H_{*}\left(V_{\prec k \succ}^{-}, V_{\langle k-1\rangle}^{-}\right)
$$

инбективен и $H_{*}\left(V_{\prec k \succ}^{-}, V_{\langle k-1\rangle}^{-}\right)$есть $\mathbb{Z}[t]$-расиирение модуля $\operatorname{Im} \lambda_{*}$.

ДокАЗАТЕльСТво. Заметим, что

$$
W^{[k]} \cap \phi^{-1}(] 0, a_{k}[)=W^{\langle k-1\rangle} \cap \phi^{-1}(] 0, a_{k}[) .
$$

C помощью вырезания можно убрать множество $\phi^{-1}(] 0, a_{k}[)$ как из $W^{[k]}$, так и из $W^{\langle k-1\rangle}$. Применяя эту процедуру к множеству $t^{s} W$ при каждом $s$, легко убедиться в том, что $H_{*}\left(V_{\prec k \succ}^{-}, V_{\langle k-1\rangle}^{-}\right)$- модуль, полученньй $\mathbb{Z}[t]-$ расширением из своего $\mathbb{Z}$-подмодуля

$$
H_{*}\left(W^{[k]} \cap \phi^{-1}\left(\left[a_{k}, b\right]\right), W^{\langle k-1\rangle} \cap \phi^{-1}\left(\left[a_{k}, b\right]\right)\right) .
$$

Последний модуль изоморфен $H_{*}\left(W^{[k]}, W^{\langle k-1\rangle}\right)$ и лемма доказана.

Теперь выражение (48) непосредственно следует из (29); достаточно заметить, что в пространстве $V^{-}$подпространство $\partial_{0} W$ совпадает с $\partial_{1} W \cdot t$. 
Итак $\widetilde{\mathscr{E}}_{*}-$ свободньй цепной комплекс над $\mathbb{Z} G_{-}$. Фильтрация $\widetilde{V}_{\langle k\rangle}^{-}$пространства $\widetilde{V}^{-}$индуцирует соответствуюшую фильтрацию в сингулярных цепных комплекcax. Эти фильтрации являются хорошими (согласно утверждениям 1 ) и 2 ) предложения 7.4), так что гомологии $H_{*}\left(\widetilde{V}^{-}\right)$изоморфны гомологиям комплекса $\widetilde{\mathscr{E}}_{*}$. Можно также вычислить простой гомотопический тип комплекса $C_{*}^{\Delta}(\widetilde{M})$ исходя из комплекса $\widetilde{\mathscr{E}}_{*}$. А именно, выберем какую-нибудь $C^{1}$-триангулящию многообразия $M$. Тогда $\widetilde{M}$ также триангулировано. Выбрав для каждого симплекса многообразия $M$ его поднятие в $\widetilde{M}$, получим естественный $\mathbb{Z} G$-базис в комплексе симплициальных цепей $C_{*}^{\Delta}(\widetilde{M})$. Таким образом, $C_{*}^{\Delta}(\widetilde{M})$ есть $\mathbb{Z} G$-комплекс. Введем базис в комплексе $\widetilde{\mathscr{E}}_{*}$, используя изоморфизмы $\widetilde{\mathscr{L}}_{k}$ (см. формулу (51)).

ПРЕДЛОЖЕнИЕ 7.6. $\mathbb{Z} G$-комплексъ $C_{*}^{\Delta}(\widetilde{M})$ u $\widetilde{\mathscr{E}}_{*} \underset{\mathbb{Z} G_{-}}{\otimes} \mathbb{Z} G$ являются просто гомотопически әквивалентными (т.е. существует гомотопическая әквивалентность ф между ними с кручением $\tau(\phi) \in W h(G)=\bar{K}_{1}(\mathbb{Z} G \mid G)$, равным нулю).

ДокАЗАТЕЛЬСТво. Выберем триангуляцию многообразия $M$ так, чтобы $V$ был симплициальным подкомплексом в $M$ и чтобы получаюшаяся триангуляция кобордизма $W$ удовлетворяла условию ( $)$ (см. доказательство предложения 5.12).

Тогда

$$
C_{*}^{\Delta}(\widetilde{M})=C_{*}^{\Delta}\left(\widetilde{V}^{-}\right) \underset{\mathbb{Z} G_{-}}{\otimes} \mathbb{Z} G .
$$

Достаточно доказать, что существует цепная гомотопическая эквивалентность $\mathbb{Z} G_{- \text {-комплексов }}$

$$
\psi: \widetilde{\mathscr{E}}_{*} \rightarrow C_{*}^{\Delta}\left(\widetilde{V}^{-}\right)
$$

такая, что $\tau(\psi) \in K_{1}\left(\mathbb{Z} G_{-} \mid H\right)$ равно нулю (напомним, что такие цепные гомотопические эквивалентности $\psi$ называются $H$-простыми). Заметим, что каждое пространство $\widetilde{V}_{\langle k\rangle}^{-}$является $\mathbb{Z} G_{-}$-инвариантным симплициальньм подкомплексом $\widetilde{V}^{-}$. Поэтому $C_{*}^{\Delta}\left(\widetilde{V}_{\langle k\rangle}^{-}\right)$образует фильтрацию в $C_{*}^{\Delta}\left(\widetilde{V}^{-}\right)$. Из предложения 7.4 следует, что эта фильтрация является хорошей. Итак, сушествует каноническая цепная гомотопическая эквивалентность

$$
\psi: \widetilde{\mathscr{E}}_{*} \rightarrow C_{*}^{\Delta}\left(\widetilde{V}^{-}\right),
$$

однозначно определенная условиями F1) и F2) (конец п. 5.4). Определим цепной комплекс $\widetilde{\mathscr{E}}(k)_{*}$ следуюшей формулой:

$$
\widetilde{\mathscr{E}}_{(k)}= \begin{cases}0, & \text { если } * \neq k, \\ \widetilde{\mathscr{E}}_{k}, & \text { если } *=k .\end{cases}
$$

Достаточно показать, что каждое цепное отображение

$$
\psi_{k}: \widetilde{\mathscr{E}}(k)_{*} \rightarrow C_{*}^{\Delta}\left(\widetilde{V}_{\langle k\rangle}^{-} / \widetilde{V}_{\langle k-1\rangle}^{-}\right)
$$

является $H$-простой гомотопической эквивалентностью. (Наше предложение выводится отсюда по индукции.) 
ЛЕмма 7.7. Цепные отображения

$$
C_{*}^{\Delta}\left(\widetilde{V}_{\prec k \succ}^{-}, \widetilde{V}_{\prec k-1 \succ}^{-}\right) \rightarrow C_{*}^{\Delta}\left(\widetilde{V}_{\prec k \succ}^{-}, \widetilde{V}_{\langle k-1\rangle}^{-}\right) \hookrightarrow C_{*}^{\Delta}\left(\widetilde{V}_{\langle k\rangle}^{-}, \widetilde{V}_{\langle k-1\rangle}^{-}\right),
$$

индуцированные включениями, являются $H$-простыми гомотопическими эквивалентностями.

ДокАЗАТЕЛЬСТво. Достаточно доказать, что для каждого $k$ включение

$$
J: C_{*}^{\Delta}\left(\widetilde{V}_{\prec k \succ}^{-}\right) \hookrightarrow C_{*}^{\Delta}\left(\widetilde{V}_{\langle k\rangle}^{-}\right)
$$

является $H$-простой гомотопической эквивалентностью.

Факторкомплекс $C_{*}\left(\widetilde{V}_{\langle k\rangle}^{-} / \widetilde{V}_{\prec k \succ}^{-}\right)$является $\mathbb{Z} G_{-}$-расширением некоторого $\mathbb{Z} H$-комплекса (заметим, что $\left.\mathbb{Z} G_{-}=(\mathbb{Z} H)[\theta]\right)$. Поэтому нам достаточно доказать, что $J / \theta$ есть $H$-простая гомотопическая эквивалентность. Цепное отображение $J / \theta$ совпадает с цепной гомотопической эквивалентностью, индуцированной включением

$$
C_{*}^{\Delta}\left(\widetilde{W}^{\prec k \succ},\left(\partial_{0} \widetilde{W}\right)^{\{\leqslant k\}}\right) \stackrel{J^{\prime}}{\hookrightarrow} C_{*}^{\Delta}\left(\widetilde{W}^{\langle k\rangle},\left(\partial_{0} \widetilde{W}\right)^{\{\leqslant k\}}\right) .
$$

Доказательство того, что $J^{\prime}$ есть простая гомотопическая эквивалентность, мы оставляем читателю в качестве несложного упражнения из теории простых гомотопических типов. (Указание: заметим, что $W^{\langle k\rangle}$ получено из $W^{\prec k \succ}$ добавлением подмножества, гомеоморфного цилиндру $\phi_{0}^{-1}\left(\left[\alpha_{k}, \alpha_{k+1}\right]\right) \times[0,1]$.)

Отображение $\psi_{k}$, очевидно, пропускается через цепное отображение

$$
\psi_{k}^{\prime}: \widetilde{\mathscr{E}}(k)_{*} \rightarrow C_{*}^{\Delta}\left(\widetilde{V}_{\prec k \succ}^{-} / \widetilde{V}_{\langle k-1\rangle}^{-}\right),
$$

и поэтому достаточно показать, что $\psi_{k}^{\prime}$ - простая гомотопическая эквивалентность. Отображение $\psi_{k}$ определяется с точностью до цепной гомотопии следующим условием: для каждого $x \in \widetilde{\mathscr{E}}(k)_{*}$ гомологический класс $\psi_{k}(x)$ есть сам $x$. Поэтому можно считать, что для каждого $p \in S_{k}(\phi)$ элемент $\psi_{k}^{\prime}([\tilde{p}])$ равен фундаментальному классу $[d(\tilde{p}), \partial d(\tilde{p})]$ пары

$$
\left(W^{\prec k \succ}, W^{\langle k-1\rangle}\right) \subset\left(V_{\prec k \succ}^{-}, \widetilde{V}_{\langle k-1\rangle}^{-}\right) .
$$

Поэтому можно предполагать, что $\psi_{k}^{\prime}$ есть $\mathbb{Z} G_{-}$-расширение, и чтобы доказать, что $\tau\left(\psi_{k}^{\prime} \mid H\right)=0$, достаточно проверить, что $\tau\left(\psi_{k}^{\prime} / \theta \mid H\right)=0$. Доказательство последнего равенства аналогично доказательству предложения 5.12 .

7.4. Замена базиса. В этом пункте мы продолжаем изучение цепного комплекса $\widetilde{\mathscr{E}}_{*}$, введенного в предыдушем пункте. Мы сравним три цепных комплекса: $\widetilde{\mathscr{E}}_{*}$, $C_{*}^{\Delta}(\widetilde{M}) \underset{\Lambda}{\otimes} \Lambda_{(\xi)}$ и $\widetilde{\mathscr{C}}_{*}(v)$. В [3] мы показали, что для каждого $f$-градиента $v$, удовлетворяюшего условию трансверсальности, $\widehat{\Lambda}_{\xi}$-комплексы $C_{*}^{\Delta}(\widetilde{M}) \underset{\Lambda}{\otimes} \widehat{\Lambda}_{\xi}$ и $\widetilde{\mathscr{C}}_{*}(v)$ гомотопически эквивалентны, и можно выбрать гомотопическую эквивалентность $\phi$ таким образом, что $\tau\left(\phi \mid U_{\xi}\right) \in K_{1}\left(\widehat{\Lambda}_{\xi} \mid U_{\xi}\right)$ равно нулю. (Кручение отображения $\phi$ в группе $\overline{K_{1}}\left(\widehat{\Lambda}_{\xi}\right)$ в общем случае не равно нулю, как мы увидим в дальнейшем.) 
Если предположить дополнительно, что $v \in \mathscr{G} \mathscr{C} \mathfrak{C} \mathscr{T}(f)$, то можно получить более сильньй результат. А именно, опираясь на предложение 7.4, мы построим цепную гомотопическую эквивалентность

$$
\tilde{\mathscr{C}}_{*}(v) \stackrel{\psi}{\longrightarrow} \widetilde{\mathscr{E}}_{*} \underset{\mathbb{Z} G_{-}}{\otimes} \Lambda_{(\xi)}
$$

$\Lambda_{(\xi)}$-комплексов (напомним, что в теореме 7.2 мы доказали, что комплекс $\widetilde{\mathscr{C}}_{*}(v)$ определен над $\left.\Lambda_{(\xi)}\right)$. Далее мы получим формулу, выражающую кручение этой гомотопической эквивалентности через гомоморфизмы $\tilde{h}_{k}$.

$$
\text { Положим } \widetilde{\mathscr{E}}_{*}=\widetilde{\mathscr{E}}_{*} \underset{\mathbb{Z} G_{-}}{\otimes} \Lambda_{(\xi)}
$$

Мы сделаем замену базиса в комплексе $\widetilde{\mathscr{E}}_{*}$, так что комплекс Новикова $\widetilde{\mathscr{C}}_{*}(v)$ будет свободным подкомплексом, порожденным некоторыми элементами нового базиса. Окажется, что факторкомплекс стягиваем.

Новьй базис вводится в определении 7.8. Каждая критическая точка $p \in S_{k}(\phi)$ определяет элемент $\{\tilde{p}\}$ нового базиса. Геометрический смысл этих элементов может быть описан следуюшим образом: образующая $[\tilde{p}]$ соответствует части $D(\tilde{p}, v) \cap$ $(\phi \circ p)^{-1}\left(\left[a_{k}, a_{k+1}\right]\right)$ нисходящего диска, а новая образующая $\{\tilde{p}\}$ соответствует всему нисходящему диску $D(\tilde{p}, v)$.

\section{Замечания о терминологии.}

1. Чтобы не перегружать обозначения, отождествим $\widetilde{\mathscr{E}}_{k}$ с $\widetilde{C}_{k}^{-}\left(u_{1}\right) \oplus \widetilde{C}_{k}^{-}(v) \oplus$ $\widetilde{C}_{k-1}^{-}\left(u_{1}\right)$. Элементы базиса в этих трех прямых слагаемых будут обозначаться символами $[\tilde{p}],[\tilde{r}],[[\tilde{q}]]$, где $p \in S_{k}\left(\phi_{1}\right), r \in S_{k}(f), q \in S_{k-1}\left(\phi_{1}\right)$. Эти элементы (в указанном порядке) образуют базис $\mathscr{B}_{k}$ в модуле $\mathscr{\mathscr { E }}_{k}$. Естественный изоморфизм, отождествляюший $\mathbb{Z} G_{-}$-подмодуль $\widetilde{C}_{k}^{-}\left(u_{1}\right) \oplus 0 \oplus 0 \subset \widetilde{\mathscr{E}}_{k}$ с подмодулем $0 \oplus 0 \oplus \widetilde{C}_{k}^{-}\left(u_{1}\right) \subset \widetilde{\mathscr{E}}_{k+1}$, будет обозначаться символом $\sigma_{k}$.

2. Граничный оператор в комплексах $\underset{\mathscr{E}}{\widetilde{\mathscr{E}}}$ и $\widetilde{\mathscr{E}}_{*} \underset{\Lambda}{\otimes} \widehat{\Lambda}_{\xi}$ будем обозначать тем же символом $\widetilde{D}_{*}$, что и граничньй оператор в комплексе $\widetilde{\mathscr{E}}_{*}$.

3. Везде, где это не ведет к неясности, мы будем опускать индексы $k+1, k$. Например, мы пишем $\widetilde{\partial}^{(1)}$ вместо $\widetilde{\partial}_{k+1}^{(1)}$ и $\widetilde{\partial}_{k}^{(1)}$. Мы часто будем опускать символ $v$ в обозначениях, так как в оставшейся части параграфа мы будем рассматривать только один $f$-градиент. Мы будем использовать эти обозначения начиная с определения 7.8 .

4. Положим

$$
\begin{gathered}
\widetilde{\widetilde{C}}_{*}\left(u_{1}\right)=\widetilde{C}_{*}\left(u_{1}\right) \underset{\mathbb{Z} H}{\otimes} \Lambda_{(\xi)}, \\
\widetilde{\widetilde{C}}_{*}(v)=\widetilde{C}_{*}(v) \underset{\mathbb{Z} H}{\otimes} \Lambda_{(\xi)} .
\end{gathered}
$$

Эти модули являются прямыми слагаемыми в $\underset{\mathscr{E}_{*}}{\widetilde{2}}$.

5. Отождествим $\Lambda_{(\xi)}$ с его образом в $\widehat{\Lambda}_{\xi}$ при каноническом вложении. Аналогичным образом, отождествим $\underset{\mathscr{E}}{\widetilde{\mathscr{E}}}$ с его образом в $\widehat{\mathscr{E}}_{*}=\widetilde{\mathscr{E}}_{*} \underset{\Lambda}{\otimes} \widehat{\Lambda}_{\xi}$. 
ОПРЕДЕЛЕНИЕ 7.8. Пусть $r \in S_{k}(f)$. Положим

$$
\{\tilde{r}\}=[\tilde{r}]-\sum_{j=0}^{\infty} \sigma \tilde{h}^{j}(\widetilde{P}([\tilde{r}])) \theta^{j} .
$$

Заметим прежде всего, что элемент $\sum_{j=0}^{\infty} \tilde{h}^{j}(a) \theta^{j}$ лежит в $\widetilde{\widetilde{C}}_{*}\left(u_{1}\right)$ (для любого $\left.a \in \widetilde{C}_{*}\left(u_{1}\right)\right)$. Поэтому $\{\tilde{r}\} \in \widetilde{\widetilde{E}}_{*}$. Далее, элемент $\{\tilde{r}\}-[\tilde{r}]$ есть линейная комбинация $\left(\right.$ с $\Lambda_{(\xi)}$-коэффициентами) элементов $[[\tilde{q}]]$, и, следовательно, элементы $[\tilde{p}],\{\tilde{r}\},[[\tilde{q}]]$ (здесь $\left.r \in S_{k}(\phi), q \in S_{k-1}\left(\phi_{1}\right), p \in S_{k}(\phi)\right)$ образуют некоторый базис $\mathscr{B}_{k}^{\prime}$ модуля $\widetilde{\mathscr{\mathscr { E }}}_{k}$, и матрища перехода от $\mathscr{B}_{k} \mathrm{~K} \mathscr{B}_{k}^{\prime}$ является произведением элементарных матриц.

Обозначим символом $\widetilde{\mathscr{C}}_{k}^{\prime}(v)$ свободньй $\Lambda_{(\xi)}$-подмодуль $\widetilde{\mathscr{E}}_{k}$, порожденньй элементами $\{\tilde{p}\}, p \in S_{k}(\phi)$. Определим гомоморфизм $\delta: \widetilde{\mathscr{C}}_{k}^{\prime}(v) \rightarrow \widetilde{\mathscr{C}}_{k-1}^{\prime}(v)$, положив

$$
\delta_{k}(\{\tilde{r}\})=\sum_{r^{\prime} \in S_{k-1}(\phi)}\left\{\tilde{r}^{\prime}\right\} \cdot \tilde{n}\left(r, r^{\prime} ; v\right) .
$$

(Таким образом, градуированный модуль $\widetilde{\mathscr{C}}_{*}^{\prime}(v)$, снабженньй дифференциалом $\delta_{*}$, изоморфен комплексу Новикова $\widetilde{\mathscr{C}}_{*}(v)$.)

ПРЕДЛОЖЕНИЕ 7.9. Матрица граничного оператора $\widetilde{D}_{k+1}: \widetilde{\mathscr{E}}_{k+1} \rightarrow \widetilde{\mathscr{E}}_{k}$ в базисах $\mathscr{B}_{k+1}^{\prime}, \mathscr{B}_{k}^{\prime}$ равна

$$
\left(\begin{array}{ccc}
\widetilde{\partial}_{k+1}^{(1)} & 0 & \mathrm{Id}-\tilde{h}_{k+1} \theta \\
0 & \delta_{k+1} & \eta_{k+1} \\
0 & 0 & \Delta_{k}
\end{array}\right)
$$

(Здесь $\eta_{k+1} u \Delta_{k}-$ некоторые гомоморфизмиц.)

ДокАЗАТЕльство. Первьй и третий столбцы не представляют трудностей. Чтобы двигаться дальше, введем несколько определений. Пусть $x \in \widehat{\mathscr{E}}_{*}$. Нам нужно будет рассматривать “часть $x$ между уровнями $a$ и $b$ функции $\widetilde{F}$ ”. Это понятие вводится в следующем определении.

ОПРЕДЕЛЕНИЕ 7.10.

1. Одночлен кольца $\widehat{\Lambda}_{\xi}$ - это элемент вида $n g, n \in \mathbb{Z}, g \in G$. Одночлен кольца $\widehat{\mathscr{E}}_{*}$ - это элемент вида $x \cdot n g$, где $n \in \mathbb{Z}, g \in G$ и $x$ - один из элементов базиса $\mathscr{B}_{*}$.

2. Bucoma $h t(\lambda)$ одночлена $\lambda=n g$ в $\widehat{\Lambda}_{\xi}$, где $n \neq 0$, равна по определению $\xi(g)$. Высота $h t(l)$ одночлена $l$ в $\widehat{\mathscr{E}}_{*}-$ это подмножество $\mathbb{R}$, определяемое следующим образом:

$$
h t(l)= \begin{cases}\xi(g)+[0,1], & \text { если } l=[\tilde{r}] \cdot n g, n \neq 0, g \in G, \\ \xi(g)+[0,1], & \text { если } l=[[\tilde{r}]] \cdot n g, n \neq 0, g \in G, \\ \xi(g)+1, & \text { если } l=[\tilde{p}] \cdot n g, n \neq 0, g \in G .\end{cases}
$$

3. Пусть $A \subset \mathbb{R}, \lambda \in \widehat{\Lambda}_{\xi}, \lambda=\sum_{g \in G} n_{g} g$. Положим $\lambda_{A}=\sum_{n_{g} \neq 0, \xi(g) \in A} n_{g} g$. Пусть $x \in \widehat{\Lambda}_{\xi}$. Определим $x_{A}$ как сумму всех одночленов $\mu$ из разложения $x$ по базису $\mathscr{B}_{k}$, удовлетворяющих $h t(\mu) \subset A$.

4. Пусть $A=\{a\}$. Мы будем писать $x_{a}$ вместо $x_{A}$.

5. Пусть $n \in \mathbb{Z}, \lambda \in \widehat{\mathscr{E}}_{*}$. Положим $\Sigma_{n} \lambda=\left(\widetilde{D}\left(\lambda_{[n, \infty[}\right)\right)_{n}$. 
ЛЕмма 7.11. Пусть $n \geqslant 0, r \in S_{k}(\phi)$. Тогда

$$
\begin{gathered}
\Sigma_{n}(\{\tilde{r}\})=\tilde{h}^{n}(\widetilde{P}([\tilde{r}])) \theta^{n}, \\
\{\tilde{r}\}_{[-n-1,-n]}=-\sigma\left(\Sigma_{-n}(\{\tilde{r}\})\right) .
\end{gathered}
$$

ДокАЗАТЕЛЬСТво очевидно.

Лемма 7.12. Пусть $A \in \mathbb{Z} G, \operatorname{supp} A \subset \xi^{-1}([n, \infty[)$, əде $n \in \mathbb{Z}$. Пусть $R=\{\tilde{r}\} A$ $u \leqslant n$. Тогда

$$
R_{[m-1, \infty[}=R_{[m, \infty[}-\sigma\left(\Sigma_{m}(R)\right)
$$

ДокАЗАТЕЛЬСтво. При $A=g, g \in G$, утверждение легко вытекает из леммы 7.11. Общий случай сводится к случаю $A=g, g \in G$, по линейности.

ЛЕмма 7.13. Для каждого $n \geqslant 0$ выполнено:

$$
(\delta\{\tilde{r}\})_{[-n-1, \infty[}=(\delta\{\tilde{r}\})_{[-n, \infty[}-\sigma\left(\Sigma_{-n}(\delta\{\tilde{r}\})\right)+\tilde{N} \tilde{h}^{n}(\widetilde{P}([\tilde{r}])) \theta^{n}
$$

ДокАЗАТЕЛЬСтво. Имеем

$(73)(\delta\{\tilde{r}\})_{[-n-1, \infty[}=\sum_{r^{\prime} \in S_{k-1}(\phi)}\{\tilde{r}\} \cdot\left(\tilde{n}\left(r, r^{\prime}\right)\right)_{[-n, \infty[}+\sum_{r^{\prime} \in S_{k-1}(\phi)}\{\tilde{r}\} \cdot\left(\tilde{n}\left(r, r^{\prime}\right)\right)_{-n-1}$.

По лемме 7.12 первый член в правой части (73) равен сумме двух первых членов в правой части (72). Второй член равен $\widetilde{N} \tilde{h}^{n}(\widetilde{P}([\tilde{r}])) \theta^{n}$, что следует из формулы (49).

Лемма 7.14. Пусть $n \geqslant 0$. Тогда

$$
\widetilde{D}\left(\{\tilde{r}\}_{[-n, \infty[}\right)=(\delta\{\tilde{r}\})_{[-n, \infty[}+\tilde{h}^{n}(\widetilde{P}([\tilde{r}])) \theta^{n} .
$$

ДокАЗАТЕЛЬСТво. Индукция по $n$. Случай $n=0$ очевиден. Предположим, что формула (74) вьполнена для некоторого целого $n$. Тогда, в частности,

$$
\widetilde{D}\left(\delta\{\tilde{r}\}_{[-n, \infty]}\right)=-\widetilde{\partial}^{(1)} \tilde{h}^{n}(\widetilde{P}([\tilde{r}])) \theta^{n}=\Sigma_{-n}(\delta\{\tilde{r}\}) .
$$

Далее,

(76) $\widetilde{D}\left(\{\tilde{r}\}_{[-n-1,-n]}\right)=\left(-\tilde{h}^{n} \widetilde{P}([\tilde{r}])+\tilde{h}^{n+1} \widetilde{P}([\tilde{r}]) \theta+\widetilde{N} \tilde{h}^{n} \widetilde{P}([\tilde{r}])+\sigma \widetilde{\partial}^{(1)} \tilde{h}^{n} \widetilde{P}([\tilde{r}])\right) \theta^{n}$ и, что вытекает из леммы 7.13,

$$
(\delta\{\tilde{r}\})_{[-n-1, \infty[}-(\delta\{\tilde{r}\})_{[-n, \infty[}=\tilde{N} \tilde{h}^{n} \widetilde{P}([\tilde{r}]) \theta^{n}+\sigma \widetilde{\partial}^{(1)} \tilde{h}^{n} \widetilde{P}([\tilde{r}]) \theta^{n}
$$

Из двух предыдущих формул легко вьвести, что утверждение (74) верно и для числа $n+1$. 
Теперь вычисление второго столбца матрицы $\widetilde{D}_{k+1}$ завершено, так как из леммы 7.14 следует, что $\widetilde{D}\{\tilde{r}\}=\delta\{\tilde{r}\}$.

7.5. Гомотопическая эквивалентность $\widetilde{\mathscr{C}}_{*}(v) \rightarrow \widetilde{\widetilde{E}}_{*}$ и ее кручение. Итак,

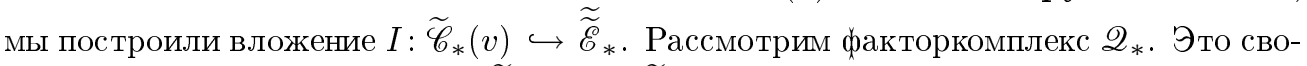
бодньй $\Lambda_{(\xi)}$-комплекс, и $\mathscr{Q}_{k}=\widetilde{\widetilde{C}}_{k}\left(u_{1}\right) \oplus \widetilde{\widetilde{C}}_{k-1}\left(u_{1}\right)$. Матрица граничного оператора $\nabla_{k}: \mathscr{Q}_{k} \rightarrow \mathscr{Q}_{k-1}$ равна

$$
\left(\begin{array}{cc}
\widetilde{\partial}_{k}^{(1)} & 1-\tilde{h}_{k} \theta \\
0 & \Delta_{k-1}
\end{array}\right)
$$

Заметим, что гомоморфизм $1-\tilde{h}_{k} \theta: \widetilde{\widetilde{C}}_{k}\left(u_{1}\right) \rightarrow \widetilde{\widetilde{C}}_{k}\left(u_{1}\right)$ является изоморфизмом, так как $\operatorname{det}\left(1-\tilde{h}_{k} \theta\right)$ имеет вид $1+\theta \xi \mathrm{c} \xi \in \mathbb{Z} H$, а такие элементы обратимы в $\Lambda_{(\xi)}$. Применяя лемму 6.1 , получаем, что $I$ - цепная гомотопическая эквивалентность и что кручение $\tau(I) \in \bar{K}_{1}\left(\Lambda_{(\xi)}\right)$ равно $\sum_{i}(-1)^{i+1} \alpha_{i}$, где $\alpha_{i}-$ класс изоморфизма $\left(1-\tilde{h}_{i} \theta\right)$. Используя специальные свойства гомоморфизма $\left(1-\tilde{h}_{k} \theta\right)$, можно еще упростить выражение для кручения $\tau(I)$. В следуюшей лемме $A$ - коммутативное кольцо с единицей, $R=A[t], S=\{P \in R \mid P=1+t Q(t)\}, \widetilde{R}=S^{-1} R, L$ - свободньй конечно-порожденный $R$-модуль, $\widetilde{L}=S^{-1} L$.

ЛЕмма 7.15. Пусть $\xi: L \rightarrow L-$ гомоморфизм. Тогда класс изоморфизма $1+$ $\xi \theta: \widetilde{L} \rightarrow \widetilde{L}$ в әруппе $\bar{K}_{1}(\widetilde{R})$ равен единице $\operatorname{det}(1+\xi \theta)$ кольца $\widetilde{R}$.

ДокаЗАтЕльство. Пусть $n=\mathrm{rk} L$. Рассмотрим класс обратимых $(n \times n)$-матриц $M$ над $\widetilde{R}$, удовлетворяюших свойству $[M]=[\operatorname{det} M]$ в $\overline{K_{1}}(\widetilde{R})$. Ясно, что этот класс содержит верхнетреугольные матрицы. Заметим также, что этот класс замкнут относительно элементарных операций. Поэтому остается доказать, что матрицу $(1+\xi \theta)$ можно привести элементарными операциями к верхнетреугольной матрице, элементы которой лежат в $\widetilde{R}$. Это несложно сделать, и мы оставим это читателю в качестве упражнения.

Итак, наше вложение $I: \widetilde{C}_{*}(v) \hookrightarrow \underset{\widetilde{\mathscr{E}}}{\widetilde{\widetilde{E}}}$ является цепной гомотопической эквивалентностью и его кручение $\tau(I) \in \bar{K}_{1}\left(\Lambda_{(\xi)}\right)$ удовлетворяет равенству

$$
\tau(I)=\left[\prod_{k}\left(\operatorname{det}\left(1-\tilde{h}_{k} \theta\right)\right)^{(-1)^{k+1}}\right] .
$$

Отметим также, что мы выбрали базисы в $\widetilde{\mathscr{C}}_{*}(v) \underset{\widetilde{\widetilde{E}}_{*}}{\widetilde{\widetilde{C}}}$ специальным образом: они построены по одному и тому же семейству поднятий критических точек $f$ до $\widetilde{M}$. Более того, мы потребовали, что поднятия критических точек $f$ до $\widetilde{M}$ принадлежали $\widetilde{W}$.

Более того, это семейство поднятий было выбрано таким образом, что все поднятия в $\widetilde{M}$ критических точек лежат в $\widetilde{W}$.

Если мы отбросим эти ограничения на семейство поднятий, то наша формула (79) для кручения останется верной, но будет утверждать только равенство двух элементов группы $\bar{K}_{1}\left(\Lambda_{(\xi)} \mid G\right)$. С помощью предложения 7.6 вьводим отсюда следующий результат. 
СлЕДСТВИЕ 7.16. Существует цепная гомотопическая әквивалентность

$$
\phi: \widetilde{\mathscr{C}}_{*}(v) \rightarrow \mathscr{C}_{*}^{\Delta}(\widetilde{M}) \underset{\Lambda}{\otimes} \Lambda_{(\xi)}
$$

такая, что

$$
\tau(\phi \mid G)=\left[\prod_{k} \operatorname{det}\left(1-\tilde{h}_{k} \theta\right)^{(-1)^{k+1}}\right] .
$$

\section{§. Доказательство основной теоремы}

Мы будем использовать обозначения предыдушего параграфа. Множество всех

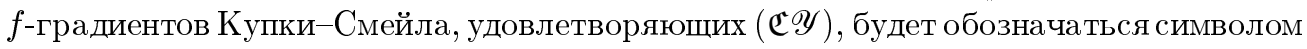
$\mathscr{G} \mathscr{K} \mathscr{S} \mathscr{C}(f)$. Мы покажем, что это множество удовлетворяет заключению теоремы. В самом деле, первое утверждение теоремы вьполнено, так как (C્Y) является $C^{0}$-открытым и плотным условием и так как $\mathscr{G} \mathscr{K} \mathscr{S} \mathscr{C}(f)$ плотно в $\mathscr{G} \mathscr{K} \mathscr{S}(f)$. Первая часть второго утверждения следует из теоремы 7.2. Остается проверить, что $\zeta_{L}(-v) \in \Lambda_{(\xi)}$ и что образ $\zeta$-функции $\zeta_{L}(-v)$ в $\bar{K}_{1}\left(\Lambda_{(\xi)} \mid G\right)$ равен элементу

$$
\left[\prod_{m} \operatorname{det}\left(1-\tilde{h}_{m} \theta\right)^{(-1)^{m+1}}\right] \text {. }
$$

Для того чтобы это проделать, нам нужно еше одно описание оператора $\tilde{h}_{m}$.

8.1. Гомологический градиентный спуск (третий вариант). Напомним, что $\phi_{1}: V_{1} \rightarrow \mathbb{R}-$ функция Морса, а $u_{1}-\delta$-разделенный $\phi_{1}$-градиент. Пусть $p \in$ $S_{m}\left(\phi_{1}\right)$. Положим

$$
R(p)=\left(D_{\delta}\left(p, u_{1}\right) \cup V_{1}^{\{\leqslant m-1\}}\right) / V_{1}^{\{\leqslant m-1\}} .
$$

Это пространство гомотопически эквивалентно сфере $S^{m}$. Пусть $k \in \mathbb{Z}$. Положим

$$
Q_{k}^{\lceil m\rceil}=\widetilde{V}_{k}^{\lceil m\rceil}(\delta) / \widetilde{V}_{k}^{\{\leqslant m-1\}}
$$

(где, как обычно, $\left.\widetilde{A}=\mathscr{P}^{-1}(A)\right)$. Группа $H$ действует на $Q_{k}^{\lceil m\rceil}$, оставляя точку $\alpha_{k}=\left[\widetilde{V}_{k}^{\{\leqslant m-1\}}\right]$ неподвижной. Поднятие $\tilde{p} \theta$ точки $p$ в $\widetilde{M}$ определяет поднятие $R(\tilde{p})$ пространства $R(p)$ в $\widetilde{M}$. Имеется следуюшее равенство:

$$
Q_{k}^{\lceil m\rceil}=\bigvee_{\substack{p \in S_{m}\left(\phi_{1}\right), h \in H, \xi(h)=k}} R(\tilde{p}) \cdot h .
$$

Образуюшая $m$-й группы гомологий пространства $R(\tilde{p})$, соответствуюшая выбранной ориентации диска $D(p, v)$, будет обозначаться символом $\rho(\tilde{p})$. Тогда $\mathbb{Z} H$-модуль $H_{*}\left(Q_{k}^{\lceil m\rceil}\right)$ является свободным, и семейство $\{\rho(\tilde{p})\}_{p \in S_{m}\left(\phi_{1}\right)}$ является базисом этого 
модуля. Несложно проверить, что оператор градиентного спуска $(-v)^{\rightsquigarrow}$ определяет непрерывное $H$-эквивариантное отображение $v \downarrow: Q_{k}^{\lceil m\rceil} \rightarrow Q_{k-1}^{\lceil m\rceil}$, переводяшее $\alpha_{k}$ в $\alpha_{k-1}$. Кроме того, следуюшая диаграмма коммутативна:

$$
\begin{array}{ccc}
H_{*}\left(\widetilde{V}_{k}^{\{\leqslant m\}}, \tilde{V}_{k}^{\{\leqslant m-1\}}\right) & \stackrel{\widetilde{\mathscr{H}}(-v)}{\longrightarrow} H_{*}\left(\tilde{V}_{k-1}^{\{\leqslant m\}}, \widetilde{V}_{k-1}^{\{\leqslant m-1\}}\right) \\
\uparrow \approx & \uparrow \approx \\
H_{*}\left(Q_{k}^{\lceil m\rceil}\right) & \stackrel{(v \downarrow)_{*}}{\longrightarrow} & H_{*}\left(Q_{k-1}^{\lceil m\rceil}\right)
\end{array}
$$

Мы оставляем читателю построение отображения $v \downarrow$ в качестве упражнения по теории гомологического градиентного спуска.

8.2. Вычисление $\zeta$-функции. Обозначим символом $\mathrm{Cl}^{[s]}(-v)$ множество всех замкнутых орбит поля $(-v)$, пересекающих множество $V^{\lceil\leqslant s\rceil}(\delta) \backslash V^{\{\leqslant s-1\}}$. Из свойства (C્Y) вытекает, что $\mathrm{Cl}(-v)$ является объединением непересекающихся подмножеств $\mathrm{Cl}^{[s]}(-v)$. Положим

$$
\eta_{s}(-v)=\sum_{\gamma \in \mathrm{Cl}^{[s]}(-v)} \varepsilon(\gamma) \frac{\pi([\gamma])}{m(\gamma)}
$$

Так как

$$
\ln \operatorname{det}\left(1-\theta \tilde{h}_{m}\right)=-\sum_{k>0} \frac{\operatorname{Tr}\left(\theta \tilde{h}_{m}\right)^{k}}{k},
$$

то достаточно будет доказать, что для любого $s$ выполнено:

$$
(-1)^{s} \sum_{k>0} \frac{\operatorname{Tr}\left(\theta \tilde{h}_{s}\right)^{k}}{k}=\eta_{s}(-v) .
$$

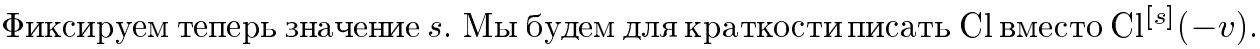
Для того чтобы доказать равенство (82), нам потребуется перевести наши исходные данные на язык теории неподвижных точек.

Мы будем говорить, что точка $a \in \beta \backslash\{\omega\}$ является $G$-неподвижсной точкой отображения $(v \downarrow)^{k}$, если $(v \downarrow)^{k}(a)=a \cdot g, g \in G$. Элемент $g \in G_{(-k)}$ однозначно определяется из вышеприведенного равенства; мы будем обозначать этот элемент символом $g(a)$. Множество всех $G$-неподвижных точек отображения $(v \downarrow)^{k}$ будет обозначаться $G F(k)$.

Множество всех $G$-неподвижных точек отображения $(v \downarrow)^{k}$ с данным $g(a)=g$ будет обозначаться символом $G F(k, g)$. Таким образом, $G F(k)=\bigsqcup_{g \in G_{(-k)}} G F(k, g)$.

По аналогии с обычной теорией неподвижных точек нетрудно определить понятия кратности $\mu(a)$ и индекса ind $a$ неподвижной точки $a \in G F(k)$.

Пусть $a \in G F(k)$. Положим $a_{i}=(v \downarrow)^{i}(a)$. Пусть $\hat{a}_{i}$ есть (единственная) точка множества $\beta$, принадлежащая $G$-орбите точки $a_{i}$. Множество всех точек $\hat{a}_{i}$ назовем квазиорбитой точки а и обозначим символом $Q(a)$; это множество конечно и число элементов в нем равно $k / \mu(a)$. 
Рассмотрим интегральную кривую $\gamma$ поля $(-v)$ в многообразии $\widetilde{M}$, удовлетворяюшую $\gamma(0)=a($ где $a \in G F(k))$. Для некоторого $T>0$ выполнено

$$
\gamma(T)=(v \downarrow)^{k}(a)=a \cdot g(a) \in \widetilde{V}_{-k} .
$$

Отображение $P \circ \gamma:[0, T] \rightarrow M$ является замкнутой орбитой поля $(-v)$. Таким образом, мы получили отображение $\Gamma: G F(k) \rightarrow \mathrm{Cl}$, образ которого состоит в точности из всех $\gamma \in \mathrm{Cl}$, удовлетворяющих условию $f_{*}([\gamma])=-k$.

Для любого $\gamma \in \mathrm{Cl}_{k}$ множество $\Gamma^{-1}(\gamma)$ является квазиорбитой некоторой точки $a \in G F_{k}$. (Заметим также, что множество $P\left(\Gamma^{-1}(\gamma)\right)$ есть пересечение орбиты $\gamma \mathrm{c}$ многообразием $V$.)

Для любой точки $a \in \Gamma^{-1}(\gamma)$ вьполнено следуюшее: $g(a)=\pi([\gamma]), \varepsilon(\gamma)=$ ind $a$, $\mu(\gamma)=\mu(a)$. Поэтому ряд

$$
\nu(-v)=\sum_{k=1}^{\infty} \frac{1}{k} \sum_{a \in G F_{k}}(\operatorname{ind} a) \cdot g(a)
$$

совпадает с $\eta_{s}(-v)$.

Чтобы доказать нашу теорему, осталось проверить, что

$$
\nu(-v)=(-1)^{s} \sum_{k>0} \frac{\operatorname{Tr}\left(\theta \tilde{h}_{s}\right)^{k}}{k} .
$$

Это равенство вытекает из следуюшей леммы.

Лemma 8.1. $\operatorname{Tr}\left(\theta \tilde{h}_{s}\right)^{k}=(-1)^{s} \sum_{a \in G F(k)}($ ind $a) \cdot g(a)$.

ДокАЗАТЕЛЬСтво. Пусть $\beta=Q_{0}^{[s]}$. Множество $G F(k, g)$ есть множество неподвижных точек композиции следуюших непрерывных отображений:

$$
\beta \stackrel{(v \downarrow)^{n}}{\longrightarrow} B_{(-k)}=\bigvee_{h \in G_{(-k)}} \beta \cdot h \stackrel{\pi_{g}}{\longrightarrow} \beta,
$$

где $\pi_{g}$ есть отображение, отправляюшее каждую компоненту букета кроме $\beta_{g}$ в точку $\omega$. Компонента же $\beta_{g}$ переводится в $\beta$ и отображение на этой компоненте совпадает с отображением $x \mapsto x g^{-1}$.

Точка $\omega \in \beta$ является неподвижной точкой композиции $\pi_{g} \circ(v \downarrow)^{k}$. Индекс точки $\omega$ равен 1. Теперь достаточно применить формулу Лефшеца-Дольда для числа неподвижных точек, и доказательство леммы 8.1 (а вместе с ней и доказательство основной теоремы) закончено. 


\section{СПИСОК ЛИТЕРАТУРЫ}

[1] Pajitnov A. V. Rationality and exponential growth properties of the boundary operators in the Novikov Complex // Math. Res. Lett. 1996. V. 3. P. 541-548.

[2] Пажитнов А. В. Рациональность граничных операторов в комплексе Новикова в случае общего положения // Алгебра и анализ. 1997. Т. 9. № 5. С. 92-139.

[3] Pazhitnov A. V. On the Novikov complex for rational Morse forms // Ann. Fac. Sci. Toulouse Math. 1995. V. 4. № 2. P. 297-338.

[4] Milnor J. Whitehead Torsion // Bull. Amer. Math. Soc. 1966. V. 72. P. 358-426.

[5] Pajitnov A. V. Surgery on the Novikov Complex // K-theory. 1996. V. 10. P. 323-412.

[6] Pajitnov A. V. Simple homotopy type of Novikov Complex for closed 1-forms and Lefschetz $\zeta$-functions of the gradient flow // e-print dg-ga/9706014 26 June 97.

[7] Hutchings M., Lee Y. J. Circle-valued Morse theory, Reidemeister torsion and SeibergWitten invariants of 3-manifolds // e-print dg-ga/9612004 3 December 1996.

[8] Pajitnov A. V. $C^{0}$-generic properties of boundary operators in Novikov complex // e-print math.DG/9812157; to appear in Adv. Math. Sci.

[9] Milnor J. Lectures on the $h$-Cobordism Theorem. Princeton: Princeton Univ. Press, 1965.

[10] Morse M. Calculus of variations in the large // American Mathematical Society Colloquium Publications. V. 18, 1934.

[11] Thom R. Sur une partition en cellules associée à une fonction sur une variété // C. R. Acad. Sci. Paris. 1949. V. 228. P. 973-975.

[12] Smale S. Generalized Poincaré's conjecture in dimensions greater than four // Ann. Math. 1961. V. 74. P. 391-406.

[13] Witten E. Supersymmetry and Morse theory // J. Differential Geom. 1982. V. 17. № 2. P. 661-692.

[14] Новиков С. П. Многозначные функции и функционалы. Аналог теории Морса // Докл. AH CCCP. 1981. T. 260. C. 31-35.

[15] Smale S. Differential dynamical systems // Bull. Amer. Math. Soc. 1967. V. 73. P. 747-817.

[16] Novikov S.P. Quasiperiodic structures in topology // Topological Methods in Modern Mathematics. Houston, TX: Publish or Perish, 1993. P. 223-235.

[17] Дольд А. Лекции по алгебраической топологии. М.: Мир, 1976.

[18] Тураев В.Г.К Кручение Райдемайстера в теории узлов // УМН. 1986. Т. 41. № 1. C. $119-182$. 